\title{
Some Generalized Dependent Aggregation Operators with Interval-Valued Intuitionistic Fuzzy Information and Their Application to Exploitation Investment Evaluation
}

\author{
Xiao-wen Qi, ${ }^{1,2}$ Chang-yong Liang, ${ }^{1,2}$ and Junling Zhang ${ }^{3}$ \\ ${ }^{1}$ School of Management, Hefei University of Technology, Hefei 230009, China \\ ${ }^{2}$ Key Laboratory of Process Optimization and Intelligent Decision-Making, Ministry of Education, Hefei 230009, China \\ ${ }^{3}$ School of Economics and Management, Zhejiang Normal University, Jinhua 321004, China \\ Correspondence should be addressed to Junling Zhang; springoasis_zhang@126.com
}

Received 28 January 2013; Accepted 21 April 2013

Academic Editor: Francisco Chiclana

Copyright (C) 2013 Xiao-wen Qi et al. This is an open access article distributed under the Creative Commons Attribution License, which permits unrestricted use, distribution, and reproduction in any medium, provided the original work is properly cited.

\begin{abstract}
We investigate multiple attribute group decision making (MAGDM) problems with arguments taking the form of interval-valued intuitionistic fuzzy numbers. In order to relieve influence of unfair arguments, a Gaussian distribution-based argument-dependent weighting method and a hybrid support-function-based argument-dependent weighting method are devised by, respectively, measuring support degrees of arguments indirectly and directly, based on which the Gaussian generalized interval-valued intuitionistic fuzzy ordered weighted averaging operator (Gaussian-GIIFOWA) and geometric operator (Gaussian-GIIFOWG), the power generalized interval-valued intuitionistic fuzzy ordered weighted averaging (P-GIIFOWA) operator and geometric (PGIIFOWA) operator are proposed to generalize a wide range of aggregation operators for decision makers to flexibly choose in decision modelling. And some desirable properties of the proposed operators are also analyzed. Further, application of an approach integrating proposed operators to exploitation investment evaluation of tourist spots has shown the effectiveness and practicality of developed methods; experimental results also verify the properties of proposed operators.
\end{abstract}

\section{Introduction}

Multiple attribute group decision making (MAGDM) is an important part of decision theories and the purpose of MAGDM is to find a desirable solution from finite alternatives by a group of experts assessing on multiple attributes with different types of decision information, such as crisp numbers [1-5], interval values [6-8], linguistic scales [9-11], and fuzzy numbers [12-17]. In order to better handle the fuzziness and uncertainty in decision process, intuitionistic fuzzy set (IFS) [18] and interval-valued intuitionistic fuzzy set (IVIFS) [19] have been introduced and increasing approaches [20-31] for MAGDM with intuitionistic fuzzy information can be found in related research literatures. Among the procedures of those MAGDM approaches, a very common information aggregation technique is the OWA [32] operator, which can provide a parameterized family of aggregation operators including the maximum, the minimum, and the average criteria. Since its appearance, the OWA operator has been developed and used in a wide range of applications in decision making and expert systems $[8,10,13,21-24,33-40]$.

The important and fundamental step of OWA operator and its extended versions is to determine the associated weights. Many researches have been carried out on this issue and useful methods have been developed under different decision environments, such as crisp numbers, interval numbers, and linguistic scales, which can be mainly classified into two categories [41]: (1) argument-independent approaches [42-48]; (2) argument-dependent approaches [4, $5,9,11,23,41,42,44,45,49-52]$. The weights derived by the argument-independent approaches are associated with particular ordered positions of the aggregated arguments and have no connection with the aggregated arguments, while the argument-dependent approaches determine the weights based on input arguments. As for the argumentdependent approaches, the prominent characteristic is that 
they can relieve the influence of unfair arguments on the aggregated results by assigning low weights to those "false" and "biased" ones. In viewing of this merit, researches on argument-dependent approaches under intuitionistic fuzzy environments and interval-valued intuitionistic fuzzy environments have started to accumulate recently, such as the linear programming-based aggregation operators with partial weight information [53], the aggregation operators [24] based on power method [54], the induced aggregation operators [55] based on Choquet integral and Dempster-Shafer theory, the power average operators [27] with trapezoidal intuitionistic fuzzy information, the generalized intuitionistic fuzzy power averaging operators [23], and the generalized dependent aggregation operators [40] for MAGDM with intuitionistic linguistic information.

Another practical and interesting research issue of applying OWA operator to MAGDM is the generalized extensions utilizing generalized means and quasiarithmetic means, which are, respectively, known as the generalized OWA (GOWA) operators $[35,56]$ and the Quasi-OWA operators [57]. And the main advantages of generalized operators are that they can generalize a wide range of aggregation operators including the average, the OWA and the OWG operators, and that they can flexibly reflect the interests and actual needs of decision makers, such as the generalized extensions [58] of induced OWA (IOWA) operator [59], the generalized weighted exponential proportional aggregation operators [4] for group decision making with crisp numbers, the expanded generalized hybrid averaging (GHA) operator [37, 60] for fuzzy multiattribute decision making environments, the induced linguistic generalized OWA (ILGOWA) operators [61], and the generalized power aggregation operators for linguistic environment [9].

As for the decision making situations with intuitionistic fuzzy information, several researches have been conducted to address suitable generalized operators recently. Zhao et al. [62] investigated extensions of GOWA operator to present the generalized intuitionistic fuzzy weighted averaging operator, generalized intuitionistic fuzzy ordered weighted averaging operator, generalized intuitionistic fuzzy hybrid averaging operator, and $\mathrm{Li}$ [36] presented another extensions of GOWA operator to accommodate intuitionistic fuzzy information. However, all the above operators are unsuitable for aggregating individual preference relations into group preference relation when some experts prefer to aggregate the variables with an inducing order. So, Xu and Xia [55] studied the induced generalized intuitionistic fuzzy Choquet integral operators and induced generalized intuitionistic fuzzy Dempster-Shafer operators. Xu and Wang [22] developed the induced generalized intuitionistic fuzzy ordered weighted averaging (IGIFOWA) operator based on the GIFOWA [62] and the I-IFOWA $[22,63]$ operators. And based on the IGOWA operator introduced by Merigó and Gil-Lafuente [58], Su et al. [21] presented another induced intuitionistic generalized fuzzy ordered weighted averaging (IG-IFOWA) operator. In addition, Zhou et al. [23] proposed a generalized operator based on the power aggregation operator and generalized mean, but the same as most researches $[9,11,24,38$, 64] that focused on extended power aggregation operators, they did not discuss construction methodology of support function in their presented operators. Comparatively, current research on generalized operators for decision making situations with interval-valued intuitionistic fuzzy information is still in its infancy; only few papers can be found in the literature. Representatively, Zhao et al. [62] further extended the GOWA operators to present some basic generalized aggregation operators for dealing with interval-valued intuitionistic fuzzy information, including the generalized interval-valued intuitionistic fuzzy weighted averaging operator, generalized interval-valued intuitionistic fuzzy ordered weighted averaging operator, and generalized interval-valued intuitionistic fuzzy hybrid average operator. Based on the Choquet integral method and Dempster-Shafer theory, Xu and Xia [55] investigated patulous induced generalized operators for aggregation of interval-valued intuitionistic fuzzy information. And most recently, Xu and Wang [22] also studied the induced version of generalized OWA operators for interval-valued intuitionistic fuzzy group decision making.

The aim of this paper is to develop some generalized argument-dependent aggregation operators more suitable for tackling with uncertainty in multiple attribute group decision making with interval-valued intuitionistic fuzzy information. Inspired by the Gaussian distribution method, we present the Gaussian generalized interval-valued intuitionistic fuzzy ordered weighted averaging (Gaussian-GIIFOWA) operator and Gaussian generalized interval-valued intuitionistic fuzzy ordered weighted geometric (Gaussian-GIIFOWG) operator; and a hybrid method is developed for construction of support degree function, based on which we further present the power generalized interval-valued intuitionistic fuzzy ordered weighted averaging (P-GIIFOWA) operator and the power generalized interval-valued intuitionistic fuzzy ordered weighted geometric (P-GIIFOWG) operator. The main advantages of these operators are that: they depend on input arguments neatly and allow arguments being aggregated to support each other so that they can relieve the influence of unfair assessments on decision results by assigning low weights to those "false" and "biased" ones; and simultaneously they can include a wide range of aggregation operators as particular cases, such as interval-valued intuitionistic fuzzy averaging (IIFA) operator, interval-valued intuitionistic fuzzy geometric (IIFG) operator, Gaussian interval-valued intuitionistic fuzzy ordered weighted geometric (GaussianIIFOWG) operator and averaging (Gaussian-IIFOWA) operator, power interval-valued intuitionistic fuzzy ordered weighted geometric (P-IIFOWG) operator and averaging (Gaussian-IIFOWA) operator, and generalized IIFA (GIIFA) operator and generalized IIFG (GIIFG) operator. Furthermore, an approach based on the proposed operators is developed and applied to solve a practical MAGDM problem concerning exploitation investment evaluation of tourist spots. This approach can give a more completely view of decision problems with decision information aggregation depending on input arguments and can also be suitable for solving other group decision making problems including supplier selection decision making, strategic management decision making, human resource management, and emergency solutions evaluation. 
The remainder of this paper is organized as follows. In Section 2, we give a concise review of fundamental concepts related to intuitionistic fuzzy sets and interval-valued intuitionistic fuzzy sets. In Section 3, we first introduce some related basic aggregation operators, and then we present two methods to obtain argument-dependent attribute weights by Gaussian distribution method and by support degree function, respectively, based on which the Gaussian-GIIFOWA operator, Gaussian-GIIFOWG operator, P-GIIFOWA operator, and P-GIIFOWG operator are presented. In addition, some desirable properties of these operators are analyzed. In Section 4, an approach for multiple attribute group decision making under interval-valued intuitionistic fuzzy environments is constructed based on the four generalized dependent aggregation operators. In Section 5, application study on exploitation investment evaluation of tourist spots is conducted to verify the validity and practicality of developed methods. Finally, conclusions are given in Section 6.

\section{Preliminaries}

In this section, we briefly review some basic concepts to facilitate future discussions.

Atanassov [18] generalized the concept of fuzzy set and defined the concept of intuitionistic fuzzy set as shown in the following Definition 1.

Definition 1 (see [18]). An intuitionistic fuzzy set (IFS) $A$ is a generalized fuzzy set and can be defined as

$$
A=\left\{\left\langle x, \mu_{A}(x), v_{A}(x)\right\rangle \mid x \in X\right\},
$$

in which $\mu_{A}$ means a membership function and $\nu_{A}$ means a nonmembership function, with the condition $0 \leq \mu_{A}(x)+$ $\nu_{A}(x) \leq 1, \mu_{A}(x), v_{A}(x) \in[0,1]$, for all $x \in X$. Particularly, $A=\mu_{A}(x)=\nu_{A}(x)$; the given IFS $A$ is degraded to an ordinary fuzzy set.

In reality, it may not be easy to identify the exact values for the membership and nonmembership degrees of an element a set. In this case, a range of values should be a more appropriate measurement to accommodate the vagueness. So, Atanassov and Gargov [19] introduced the notion of intervalvalued intuitionistic fuzzy set (IVIFS).

Definition 2 (see [19]). An interval-valued intuitionistic fuzzy set (IVIFS) $\widetilde{A}$ in $X$ can be defined as

$$
\begin{aligned}
\widetilde{A} & =\left\{\left\langle x, \widetilde{\mu}_{\widetilde{A}}(x), \widetilde{v}_{\widetilde{A}}(x)\right\rangle \mid x \in X\right\} \\
& =\left\{\left\langle x,\left[\mu_{\widetilde{A}}^{L}(x), \mu_{\widetilde{A}}^{U}(x)\right],\left[\nu_{\widetilde{A}}^{L}(x), \nu_{\widetilde{A}}^{U}(x)\right]\right\rangle \mid x \in X\right\},
\end{aligned}
$$

where $0 \leq \mu_{\widetilde{A}}^{L}(x) \leq \mu_{\widetilde{A}}^{U}(x) \leq 1,0 \leq \nu_{\widetilde{A}}^{L}(x) \leq \nu_{\widetilde{A}}^{U}(x) \leq 1$, $0 \leq \mu_{\widetilde{A}}^{U}(x)+\nu_{\widetilde{A}}^{U}(x) \leq 1$ for all $x \in X$.

Similarly, the intervals $\widetilde{\mu}_{\widetilde{A}}(x)$ and $\widetilde{v}_{\widetilde{A}}(x)$ denote the membership and non-membership of an element a set.

If each of the intervals $\widetilde{\mu}_{\widetilde{A}}(x)$ and $\widetilde{\nu}_{\widetilde{A}}(x)$ contains only one value for each $x \in X$, we have

$$
\tilde{\mu}_{\widetilde{A}}(x)=\mu_{\widetilde{A}}^{L}(x)=\mu_{\widetilde{A}}^{U}(x), \quad \widetilde{\nu}_{\widetilde{A}}(x)=\nu_{\widetilde{A}}^{L}(x)=\nu_{\widetilde{A}}^{U}(x) .
$$

Then, the given IVIFS $\widetilde{A}$ is degraded to an ordinary IFS.

In order to aggregate interval-valued intuitionistic fuzzy information, $\mathrm{Xu}[65]$ defined the following relations and basic operations.

Definition 3 (see [65]). Let $\widetilde{\alpha}=([a, b],[c, d]), \widetilde{\alpha}_{1}=\left(\left[a_{1}, b_{1}\right]\right.$, $\left.\left[c_{1}, d_{1}\right]\right), \widetilde{\alpha}_{2}=\left(\left[a_{2}, b_{2}\right],\left[c_{2}, d_{2}\right]\right)$ be interval-valued intuitionistic fuzzy numbers (IVIFNs), then

(1) $\tilde{\alpha}_{1} \oplus \widetilde{\alpha}_{2}=\left(\left[a_{1}+a_{2}-a_{1} a_{2}, b_{1}+b_{2}-b_{1} b_{2}\right],\left[c_{1} c_{2}, d_{1} d_{2}\right]\right)$;

(2) $\widetilde{\alpha}_{1} \otimes \widetilde{\alpha}_{2}=\left(\left[a_{1} a_{2}, b_{1} b_{2}\right],\left[c_{1}+c_{2}-c_{1} c_{2}, d_{1}+d_{2}-d_{1} d_{2}\right]\right)$;

(3) $\lambda \widetilde{\alpha}=\left(\left[1-(1-a)^{\lambda}, 1-(1-b)^{\lambda}\right],\left[c^{\lambda}, d^{\lambda}\right]\right)$;

(4) $\tilde{\alpha}^{\lambda}=\left(\left[a^{\lambda}, b^{\lambda}\right],\left[1-(1-c)^{\lambda}, 1-(1-d)^{\lambda}\right]\right)$.

Usually, the following normalized distance measure formulae listed in Definition 4 can be introduced to calculate the distance of IVIFSs.

Definition 4. Suppose that two interval-valued intuitionistic fuzzy sets (IVIFSs) $\widetilde{A}$ and $\widetilde{B}$ in $X$ can be defined as

$$
\begin{aligned}
\widetilde{A} & =\left\{\left\langle x_{i}, \widetilde{\mu}_{\widetilde{A}}\left(x_{i}\right), \widetilde{v}_{\widetilde{A}}\left(x_{i}\right)\right\rangle \mid x_{i} \in X\right\} \\
& =\left\{\left\langle x_{i},\left[\mu_{\widetilde{A}}^{L}\left(x_{i}\right), \mu_{\widetilde{A}}^{U}\left(x_{i}\right)\right],\left[\nu_{\widetilde{A}}^{L}\left(x_{i}\right), \nu_{\widetilde{A}}^{U}\left(x_{i}\right)\right]\right\rangle \mid x_{i} \in X\right\}, \\
\widetilde{B} & =\left\{\left\langle x_{i}, \widetilde{\mu}_{\widetilde{B}}\left(x_{i}\right), \widetilde{v}_{\widetilde{B}}\left(x_{i}\right)\right\rangle \mid x_{i} \in X\right\} \\
& =\left\{\left\langle x_{i},\left[\mu_{\widetilde{B}}^{L}\left(x_{i}\right), \mu_{\widetilde{B}}^{U}\left(x_{i}\right)\right],\left[\nu_{\widetilde{B}}^{L}\left(x_{i}\right), v_{\widetilde{B}}^{U}\left(x_{i}\right)\right]\right\rangle \mid x_{i} \in X\right\} ;
\end{aligned}
$$

then we can have

(1) the normalized Euclidean distance measure:

$$
\begin{aligned}
D_{1}(\widetilde{A}, \widetilde{B}) & \\
=\left(\frac{1}{6 n} \sum_{i=1}^{n}[\right. & \left(\mu_{\widetilde{A}}^{L}\left(x_{i}\right)-\mu_{\widetilde{B}}^{L}\left(x_{i}\right)\right)^{2} \\
& +\left(\mu_{\widetilde{A}}^{U}\left(x_{i}\right)-\mu_{\widetilde{B}}^{U}\left(x_{i}\right)\right)^{2} \\
& +\left(\nu_{\widetilde{A}}^{L}\left(x_{i}\right)-\nu_{\widetilde{B}}^{L}\left(x_{i}\right)\right)^{2} \\
& +\left(\nu_{\widetilde{A}}^{U}\left(x_{i}\right)-\nu_{\widetilde{B}}^{U}\left(x_{i}\right)\right)^{2} \\
& +\left(\pi_{\widetilde{A}}^{L}\left(x_{i}\right)-\pi_{\widetilde{B}}^{L}\left(x_{i}\right)\right)^{2} \\
& \left.\left.+\left(\pi_{\widetilde{A}}^{U}\left(x_{i}\right)-\pi_{\widetilde{B}}^{U}\left(x_{i}\right)\right)^{2}\right]\right)^{1 / 2} ;
\end{aligned}
$$


(2) the normalized Hamming distance measure:

$$
\begin{aligned}
D_{2}(\widetilde{A}, \widetilde{B}) & \\
=\frac{1}{6 n} \sum_{i=1}^{n} & \left|\mu_{\widetilde{A}}^{L}\left(x_{i}\right)-\mu_{\widetilde{B}}^{L}\left(x_{i}\right)\right| \\
& +\left|\mu_{\widetilde{A}}^{U}\left(x_{i}\right)-\mu_{\widetilde{B}}^{U}\left(x_{i}\right)\right| \\
& +\left|\nu_{\widetilde{A}}^{L}\left(x_{i}\right)-\nu_{\widetilde{B}}^{L}\left(x_{i}\right)\right| \\
& +\left|\nu_{\widetilde{A}}^{U}\left(x_{i}\right)-\nu_{\widetilde{B}}^{U}\left(x_{i}\right)\right| \\
& +\left|\pi_{\widetilde{A}}^{L}\left(x_{i}\right)-\pi_{\widetilde{B}}^{L}\left(x_{i}\right)\right| \\
& +\left|\pi_{\widetilde{A}}^{U}\left(x_{i}\right)-\pi_{\widetilde{B}}^{U}\left(x_{i}\right)\right| ;
\end{aligned}
$$

(3) the normalized Hausdorff distance measure:

$$
\begin{aligned}
D_{3}(\widetilde{A}, \widetilde{B}) & \\
=\frac{1}{n} \sum_{i=1}^{n} \max \{ & \left\{\left|\mu_{\widetilde{A}}^{L}\left(x_{i}\right)-\mu_{\widetilde{B}}^{L}\left(x_{i}\right)\right|,\right. \\
& \left|\mu_{\widetilde{A}}^{U}\left(x_{i}\right)-\mu_{\widetilde{B}}^{U}\left(x_{i}\right)\right|, \\
& \left|\nu_{\widetilde{A}}^{L}\left(x_{i}\right)-\nu_{\widetilde{B}}^{L}\left(x_{i}\right)\right|, \\
& \left|\nu_{\widetilde{A}}^{U}\left(x_{i}\right)-\nu_{\widetilde{B}}^{U}\left(x_{i}\right)\right|, \\
& \left|\pi_{\widetilde{A}}^{L}\left(x_{i}\right)-\pi_{\widetilde{B}}^{L}\left(x_{i}\right)\right|, \\
& \left.\left|\pi_{\widetilde{A}}^{U}\left(x_{i}\right)-\pi_{\widetilde{B}}^{U}\left(x_{i}\right)\right|\right\} .
\end{aligned}
$$

In order to rank alternatives, it is necessary to consider how to compare two interval-valued intuitionistic fuzzy numbers, so $\mathrm{Xu}$ [66] devised an approach to compare two IVIFNs based on the concepts of score function and accuracy function.

Definition 5 (see [66]). For any three IVIFNs $\widetilde{\alpha}=\left(\left[\mu^{L}, \mu^{U}\right]\right.$, $\left.\left[v^{L}, v^{U}\right]\right), \widetilde{\alpha}_{1}=\left(\left[\mu_{1}^{L}, \mu_{1}^{U}\right],\left[v_{1}^{L}, v_{1}^{U}\right]\right)$, and $\widetilde{\alpha}_{2}=\left(\left[\mu_{2}^{L}, \mu_{2}^{U}\right],\left[v_{2}^{L}\right.\right.$, $\left.\left.\nu_{2}^{U}\right]\right)$, score function can be defined as $s(\widetilde{\alpha})=(1 / 2)\left(\mu^{L}+\right.$ $\left.\mu^{U}-v^{L}-\nu^{U}\right)$, accuracy function can be defined as $h(\widetilde{\alpha})=$ $(1 / 2)\left(\mu^{L}+\mu^{U}+\nu^{L}+\nu^{U}\right)$, and

$$
\begin{aligned}
& \text { if } s\left(\widetilde{\alpha}_{1}\right)<s\left(\widetilde{\alpha}_{2}\right) \text {, then } \widetilde{\alpha}_{1} \text { is smaller than } \widetilde{\alpha}_{2}, \widetilde{\alpha}_{1}<\widetilde{\alpha}_{2} ; \\
& \text { if } s\left(\widetilde{\alpha}_{1}\right)>s\left(\widetilde{\alpha}_{2}\right) \text {, then } \widetilde{\alpha}_{1} \text { is greater than } \widetilde{\alpha}_{2}, \widetilde{\alpha}_{1}>\widetilde{\alpha}_{2} ; \\
& \text { if } s\left(\widetilde{\alpha}_{1}\right)=s\left(\widetilde{\alpha}_{2}\right) \text {, then }
\end{aligned}
$$$$
\text { if } h\left(\widetilde{\alpha}_{1}\right)<h\left(\widetilde{\alpha}_{2}\right) \text {, then } \widetilde{\alpha}_{1} \text { is smaller than } \widetilde{\alpha}_{2}, \widetilde{\alpha}_{1}<
$$$$
\tilde{\alpha}_{2} \text {; }
$$$$
\text { if } h\left(\widetilde{\alpha}_{1}\right)>h\left(\widetilde{\alpha}_{2}\right) \text {, then } \widetilde{\alpha}_{1} \text { is greater than } \widetilde{\alpha}_{2}, \widetilde{\alpha}_{1}>
$$
$\tilde{\alpha}_{2}$;

if $h\left(\widetilde{\alpha}_{1}\right)=h\left(\widetilde{\alpha}_{2}\right)$, then $\widetilde{\alpha}_{1}$ and $\widetilde{\alpha}_{2}$ represent the same information, denoted by $\widetilde{\alpha}_{1}=\widetilde{\alpha}_{2}$.

\section{Proposed Generalized Dependent Interval-Valued Intuitionistic Fuzzy Ordered Weighted Aggregation Operators}

3.1. Basic Operators. Up to now, some useful operators have been proposed for aggregating the interval-valued intuitionistic fuzzy information. The most commonly used two operators for aggregating interval-valued intuitionistic fuzzy arguments are the interval-valued intuitionistic fuzzy weighted averaging (IIFWA) operator and geometric (IIFWG) operator as defined by $\mathrm{Xu}$ [65] in the following definitions.

Definition 6 (see [65]). An interval-valued intuitionistic fuzzy weighted averaging (IIFWA) operator of dimension $n$ is a mapping IIFWA: $\Omega^{n} \rightarrow \Omega$, which has an argument associated vector $\omega=\left(\omega_{1}, \omega_{2}, \ldots, \omega_{n}\right)^{T}$ with $\omega_{j} \in[0,1]$ and $\sum_{j=1}^{n} \omega_{j}=1$, such that

$$
\operatorname{IIFWA}_{\omega}\left(\tilde{\alpha}_{1}, \tilde{\alpha}_{2}, \ldots, \tilde{\alpha}_{n}\right)=\omega_{1} \tilde{\alpha}_{1} \oplus \omega_{2} \widetilde{\alpha}_{2} \oplus \cdots \oplus \omega_{n} \widetilde{\alpha}_{n} .
$$

Let $\widetilde{a}_{i}=\left(\left[a_{i}, b_{i}\right],\left[c_{i}, d_{i}\right]\right)(i=1,2, \ldots, n)$ be a collection of interval-valued intuitionistic fuzzy numbers, then their aggregated value by using the IIFWA operator can be shown as

$$
\begin{aligned}
& \operatorname{IIFWA}_{\omega}\left(\widetilde{\alpha}_{1}, \widetilde{\alpha}_{2}, \ldots, \widetilde{\alpha}_{n}\right) \\
&=\left(\left[1-\prod_{j=1}^{n}\left(1-a_{j}\right)^{\omega_{j}}, 1-\prod_{j=1}^{n}\left(1-b_{j}\right)^{\omega_{j}}\right],\right. \\
& {\left.\left[\prod_{j=1}^{n} c_{j}^{\omega_{j}}, \prod_{j=1}^{n} d_{j}^{\omega_{j}}\right]\right) . }
\end{aligned}
$$

Particularly, when $\omega=(1 / n, 1 / n, \ldots, 1 / n)^{T}$, the IIFWA operator reduces to the interval-valued intuitionistic fuzzy averaging (IIFA) operator; that is,

$$
\begin{aligned}
& \operatorname{IIFWA}_{\omega}\left(\widetilde{\alpha}_{1}, \widetilde{\alpha}_{2}, \ldots, \widetilde{\alpha}_{n}\right) \\
& =\frac{1}{n} \widetilde{\alpha}_{1} \oplus \frac{1}{n} \widetilde{\alpha}_{2} \oplus \cdots \oplus \frac{1}{n} \widetilde{\alpha}_{n} \\
& =\left(\left[1-\prod_{j=1}^{n}\left(1-a_{j}\right)^{1 / n}, 1-\prod_{j=1}^{n}\left(1-b_{j}\right)^{1 / n}\right],\right. \\
& \left.=\left[\prod_{j=1}^{n} c_{j}^{1 / n}, \prod_{j=1}^{n} d_{j}^{1 / n}\right]\right) \\
& =\operatorname{IIFA}\left(\widetilde{\alpha}_{1}, \widetilde{\alpha}_{2}, \ldots, \widetilde{\alpha}_{n}\right) .
\end{aligned}
$$

Definition 7 (see [65]). An interval-valued intuitionistic fuzzy weighted geometric (IIFWG) operator of dimension $n$ is a mapping IIFWG: $\Omega^{n} \rightarrow \Omega$, which has an argument 
associated vector $\omega=\left(\omega_{1}, \omega_{2}, \ldots, \omega_{n}\right)^{T}$ with $\omega_{j} \in[0,1]$ and $\sum_{j=1}^{n} \omega_{j}=1$, such that

$$
\begin{aligned}
& \operatorname{IIFWG}_{\omega}\left(\widetilde{\alpha}_{1}, \widetilde{\alpha}_{2}, \ldots, \widetilde{\alpha}_{n}\right) \\
&=\widetilde{\alpha}_{1}^{\omega_{1}} \otimes \widetilde{\alpha}_{2}^{\omega_{2}} \otimes \cdots \otimes \widetilde{\alpha}_{n}^{\omega_{n}} \\
&=\left(\left[\prod_{j=1}^{n} a_{j}^{\omega_{j}}, \prod_{j=1}^{n} b_{j}^{\omega_{j}}\right],\right. \\
& {\left.\left[1-\prod_{j=1}^{n}\left(1-c_{j}\right)^{\omega_{j}}, 1-\prod_{j=1}^{n}\left(1-d_{j}\right)^{\omega_{j}}\right]\right) . }
\end{aligned}
$$

Particularly, when $\omega=(1 / n, 1 / n, \ldots, 1 / n)^{T}$, the IIFWG operator reduces to the interval-valued intuitionistic fuzzy geometric (IIFG) operator; that is,

$$
\begin{aligned}
\operatorname{IIFWG}_{\omega} & \left(\widetilde{\alpha}_{1}, \widetilde{\alpha}_{2}, \ldots, \widetilde{\alpha}_{n}\right) \\
= & \widetilde{\alpha}_{1}^{1 / n} \otimes \widetilde{\alpha}_{2}^{1 / n} \otimes \cdots \otimes \widetilde{\alpha}_{n}^{1 / n} \\
= & \left(\left[\prod_{j=1}^{n} a_{j}^{1 / n}, \prod_{j=1}^{n} b_{j}^{1 / n}\right],\right. \\
& {\left.\left[1-\prod_{j=1}^{n}\left(1-c_{j}\right)^{1 / n}, 1-\prod_{j=1}^{n}\left(1-d_{j}\right)^{1 / n}\right]\right) } \\
= & \operatorname{IIFG}\left(\widetilde{\alpha}_{1}, \widetilde{\alpha}_{2}, \ldots, \widetilde{\alpha}_{n}\right) .
\end{aligned}
$$

Considering ordered positions of interval-valued intuitionistic fuzzy arguments rather than weighting the intervalvalued intuitionistic fuzzy arguments themselves, $\mathrm{Xu}$ and Chen [67] proposed an interval-valued intuitionistic fuzzy ordered weighted averaging (IIFOWA) operator and an interval-valued intuitionistic fuzzy ordered weighted geometric (IIFOWG) operator, as shown in the following definitions.

Definition 8 (see [67]). Let $\left(\widetilde{\alpha}_{1}, \widetilde{\alpha}_{2}, \ldots, \widetilde{\alpha}_{n}\right)$ be a collection of interval-valued intuitionistic fuzzy arguments, and $\widetilde{\alpha}_{j}=\left(\left[a_{j}, b_{j}\right],\left[c_{j}, d_{j}\right]\right)$. The interval-valued intuitionistic fuzzy ordered weighted averaging (IIFOWA) operator of dimension $n$ is a mapping IIFOWA: $R^{n} \rightarrow R$, which has an associated weight vector $w=\left(w_{1}, w_{2}, \ldots, w_{n}\right)^{T}, \sum_{j=1}^{n} w_{j}=1$ and $w_{j} \in[0,1]$; then

$$
\operatorname{IIFOWA}_{w}\left(\widetilde{\alpha}_{1}, \widetilde{\alpha}_{2}, \ldots, \widetilde{\alpha}_{n}\right)=w_{1} \widetilde{\beta}_{1} \oplus w_{2} \widetilde{\beta}_{2} \oplus \cdots \oplus w_{n} \widetilde{\beta}_{n},
$$

where $\left(\widetilde{\beta}_{1}, \widetilde{\beta}_{2}, \ldots, \widetilde{\beta}_{n}\right)$ is a permutation of $\left(\widetilde{\alpha}_{1}, \widetilde{\alpha}_{2}, \ldots, \widetilde{\alpha}_{n}\right)$, with $\widetilde{\beta}_{j-1} \geq \widetilde{\beta}_{j}$ for all $j$.
Particularly, when $w=(1 / n, 1 / n, \ldots, 1 / n)^{T}$, the IIFOWA operator reduces to the IIFA operator; that is,

$$
\begin{aligned}
& \operatorname{IIFOWA}_{w}\left(\widetilde{\alpha}_{1}, \widetilde{\alpha}_{2}, \ldots, \widetilde{\alpha}_{n}\right) \\
& =\frac{1}{n} \widetilde{\beta}_{1} \oplus \frac{1}{n} \widetilde{\beta}_{2} \oplus \cdots \oplus \frac{1}{n} \widetilde{\beta}_{n} \\
& =\operatorname{IIFA}_{w}\left(\widetilde{\alpha}_{1}, \widetilde{\alpha}_{2}, \ldots, \widetilde{\alpha}_{n}\right) .
\end{aligned}
$$

Definition 9 (see [67]). Let $\left(\widetilde{\alpha}_{1}, \widetilde{\alpha}_{2}, \ldots, \widetilde{\alpha}_{n}\right)$ be a collection of interval-valued intuitionistic fuzzy arguments, and $\widetilde{\alpha}_{j}=$ $\left(\left[a_{j}, b_{j}\right],\left[c_{j}, d_{j}\right]\right)$. The IIFOWG operator of dimension $n$ is a mapping IIFOWG: $R^{n} \rightarrow R$, which has an associated weight vector $w=\left(w_{1}, w_{2}, \ldots, w_{n}\right)^{T}, \sum_{j=1}^{n} w_{j}=1$ and $w_{j} \in[0,1]$; then

$$
\operatorname{IIFOWG}_{w}\left(\widetilde{\alpha}_{1}, \widetilde{\alpha}_{2}, \ldots, \widetilde{\alpha}_{n}\right)=\widetilde{\beta}_{1}^{w_{1}} \otimes \widetilde{\beta}_{2}^{w_{2}} \otimes \cdots \otimes \widetilde{\beta}_{n}^{w_{n}},
$$

where $\left(\widetilde{\beta}_{1}, \widetilde{\beta}_{2}, \ldots, \widetilde{\beta}_{n}\right)$ is a permutation of $\left(\widetilde{\alpha}_{1}, \widetilde{\alpha}_{2}, \ldots, \widetilde{\alpha}_{n}\right)$, with $\widetilde{\beta}_{j-1} \geq \widetilde{\beta}_{j}$ for all $j$.

Particularly, when $w=(1 / n, 1 / n, \ldots, 1 / n)^{T}$, the IIFOWG operator reduces to the IIFG operator; that is,

$$
\begin{aligned}
& \operatorname{IIFOWG}_{w}\left(\widetilde{\alpha}_{1}, \widetilde{\alpha}_{2}, \ldots, \widetilde{\alpha}_{n}\right) \\
& =\widetilde{\beta}_{1}^{1 / n} \otimes \widetilde{\beta}_{2}^{1 / n} \otimes \cdots \otimes \widetilde{\beta}_{n}^{1 / n} \\
& =\operatorname{IIFG}_{w}\left(\widetilde{\alpha}_{1}, \widetilde{\alpha}_{2}, \ldots, \widetilde{\alpha}_{n}\right) .
\end{aligned}
$$

From another important and practical aspect, Yager [56] defined a generalized version of OWA operators as the generalized ordered weighted averaging (GOWA) operator; then Zhao et al. [62] extended it to the situations where input arguments are IVIFNs and presented a generalized interval-valued intuitionistic fuzzy ordered weighted averaging (GIIFOWA) operator and geometric (GIIFOWG) operator as defined in Definitions 10 and 11.

Definition 10 (see [62]). Let $\left(\widetilde{\alpha}_{1}, \widetilde{\alpha}_{2}, \ldots, \widetilde{\alpha}_{n}\right)$ be a collection of interval-valued intuitionistic fuzzy arguments, and $\widetilde{\alpha}_{j}=$ $\left(\left[a_{j}, b_{j}\right],\left[c_{j}, d_{j}\right]\right)$. The GIIFOWA operator of dimension $n$ is a mapping GIIFOWA: $R^{n} \rightarrow R$, which has an associated weight vector $w=\left(w_{1}, w_{2}, \ldots, w_{n}\right)^{T}, \sum_{j=1}^{n} w_{j}=1$ and $w_{j} \epsilon$ $[0,1], \lambda>0$; then

$$
\begin{aligned}
\operatorname{GIIFOWA}_{\lambda}\left(\widetilde{\alpha}_{1}, \widetilde{\alpha}_{2}, \ldots, \widetilde{\alpha}_{n}\right) \\
=\left(\oplus_{j=1}^{n}\left(w_{j} \widetilde{\beta}_{j}^{\lambda}\right)\right)^{1 / \lambda} \\
=\left(\left[\left(1-\prod_{j=1}^{n}\left(1-a_{\tilde{\beta}(j)}^{\lambda}\right)^{w_{j}}\right)^{1 / \lambda},\right.\right. \\
\left.\quad\left(1-\prod_{j=1}^{n}\left(1-b_{\tilde{\beta}(j)}^{\lambda}\right)^{w_{j}}\right)^{1 / \lambda}\right],
\end{aligned}
$$




$$
\begin{aligned}
& {\left[1-\left(1-\prod_{j=1}^{n}\left(1-\left(1-c_{\widetilde{\beta}(j)}\right)^{\lambda}\right)^{w_{j}}\right)^{1 / \lambda},\right.} \\
& \left.\left.1-\left(1-\prod_{j=1}^{n}\left(1-\left(1-d_{\widetilde{\beta}(j)}\right)^{\lambda}\right)^{w_{j}}\right)^{1 / \lambda}\right]\right),
\end{aligned}
$$

where $\left(\widetilde{\beta}_{1}, \widetilde{\beta}_{2}, \ldots, \widetilde{\beta}_{n}\right)$ is a permutation of $\left(\widetilde{\alpha}_{1}, \widetilde{\alpha}_{2}, \ldots, \widetilde{\alpha}_{n}\right)$, with $\widetilde{\beta}_{j-1} \geq \widetilde{\beta}_{j}$ for all $j$.

If $w=(1 / n, 1 / n, \ldots, 1 / n)^{T}$, then the GIIFOWA operator reduces to the GIIFA operator; that is,

$$
\begin{aligned}
\operatorname{GIIFOWA}_{\lambda}\left(\widetilde{\alpha}_{1}, \widetilde{\alpha}_{2}, \ldots, \widetilde{\alpha}_{n}\right) & =\left(\bigoplus_{j=1}^{n}\left(\frac{1}{n} \widetilde{\beta}_{j}^{\lambda}\right)\right)^{1 / \lambda} \\
& =\operatorname{GIIFA}_{\lambda}\left(\widetilde{\alpha}_{1}, \widetilde{\alpha}_{2}, \ldots, \widetilde{\alpha}_{n}\right) .
\end{aligned}
$$

Definition 11 (see [62]). Let $\left(\widetilde{\alpha}_{1}, \widetilde{\alpha}_{2}, \ldots, \widetilde{\alpha}_{n}\right)$ be a collection of interval-valued intuitionistic fuzzy arguments, and $\widetilde{\alpha}_{j}=$ $\left(\left[a_{j}, b_{j}\right],\left[c_{j}, d_{j}\right]\right)$. The GIIFOWG operator of dimension $n$ is a mapping GIIFOWG: $R^{n} \rightarrow R$, which has an associated weight vector $w=\left(w_{1}, w_{2}, \ldots, w_{n}\right)^{T}, \sum_{j=1}^{n} w_{j}=1$ and $w_{j} \in$ $[0,1], \lambda>0$; then

$$
\begin{aligned}
& \operatorname{GIIFOWG}_{\lambda}\left(\widetilde{\alpha}_{1}, \widetilde{\alpha}_{2}, \ldots, \widetilde{\alpha}_{n}\right) \\
& =\frac{1}{\lambda}\left(\bigotimes_{j=1}^{n}\left(\lambda \widetilde{\beta}_{j}\right)^{w_{j}}\right) \\
& =\left(\left[1-\left(1-\prod_{j=1}^{n}\left(1-\left(1-a_{\widetilde{\beta}(j)}\right)^{\lambda}\right)^{w_{j}}\right)^{1 / \lambda},\right.\right. \\
& \left.1-\left(1-\prod_{j=1}^{n}\left(1-\left(1-b_{\widetilde{\beta}(j)}\right)^{\lambda}\right)^{w_{j}}\right)^{1 / \lambda}\right], \\
& {\left[\left(1-\prod_{j=1}^{n}\left(1-c_{\tilde{\beta}(j)}^{\lambda}\right)^{w_{j}}\right)^{1 / \lambda}\right.} \\
& \left.\left.\left(1-\prod_{j=1}^{n}\left(1-d_{\widetilde{\beta}(j)}^{\lambda}\right)^{w_{j}}\right)^{1 / \lambda}\right]\right),
\end{aligned}
$$

where $\left(\widetilde{\beta}_{1}, \widetilde{\beta}_{2}, \ldots, \widetilde{\beta}_{n}\right)$ is a permutation of $\left(\widetilde{\alpha}_{1}, \widetilde{\alpha}_{2}, \ldots, \widetilde{\alpha}_{n}\right)$, with $\widetilde{\beta}_{j-1} \geq \widetilde{\beta}_{j}$ for all $j$.
If $w=(1 / n, 1 / n, \ldots, 1 / n)^{T}$, then the GIIFOWG operator reduces to the GIIFG operator; that is,

$$
\begin{aligned}
\operatorname{GIIFOWG}_{\lambda}\left(\widetilde{\alpha}_{1}, \widetilde{\alpha}_{2}, \ldots, \widetilde{\alpha}_{n}\right) & =\frac{1}{\lambda}\left({\underset{j=1}{\otimes}}_{j}\left(\lambda \widetilde{\beta}_{j}\right)^{1 / n}\right) \\
& =\operatorname{GIIFG}_{\lambda}\left(\widetilde{\alpha}_{1}, \widetilde{\alpha}_{2}, \ldots, \widetilde{\alpha}_{n}\right) .
\end{aligned}
$$

From Definition 8 to Definition 11, it can be seen that one important and basic step of interval-valued intuitionistic fuzzy ordered weighted aggregation operators and generalized versions is to determine the associated weights. In the following subsections, we will focus on investigating argument-dependent operators in which the associated weights can be determined objectively only depending on the interval-valued intuitionistic fuzzy input arguments.

3.2. Proposed Gaussian Generalized Interval-Valued Intuitionistic Fuzzy Aggregation Operators. According to the basic operational rules listed in Definition 3 and IIFWA operator in Definition 6 for aggregating IVIFNs, here we can naturally define mean value of a set of IVIFNs as shown in the following definition. Obviously, the mean value $\tilde{\mu}$ is still an IVIFN.

Definition 12. Let $\left(\widetilde{\alpha}_{1}, \widetilde{\alpha}_{2}, \ldots, \widetilde{\alpha}_{n}\right)$ be a collection of interval-valued intuitionistic fuzzy arguments, where $\tilde{\alpha}_{j}=\left(\left[a_{j}\right.\right.$, $\left.\left.b_{j}\right],\left[c_{j}, d_{j}\right]\right)$. Let $\tilde{\mu}$ be the mean value of $\left(\widetilde{\alpha}_{1}, \widetilde{\alpha}_{2}, \ldots, \widetilde{\alpha}_{n}\right)$, and $\tilde{\mu}=\left(\left[a_{\mu}, b_{\mu}\right],\left[c_{\mu}, d_{\mu}\right]\right)$; then $\tilde{\mu}$ can be obtained by IIFWA operator with $\omega=(1 / n, 1 / n, \ldots, 1 / n)^{T}$, where

$$
\begin{array}{cc}
a_{\mu}=1-\prod_{j=1}^{n}\left(1-a_{j}\right)^{1 / n}, & b_{\mu}=1-\prod_{j=1}^{n}\left(1-b_{j}\right)^{1 / n}, \\
c_{\mu}=\prod_{j=1}^{n} c_{j}^{1 / n}, & d_{\mu}=\prod_{j=1}^{n} d_{j}^{1 / n} .
\end{array}
$$

Definition 13 (see [68]). Let $\left(\widetilde{\alpha}_{1}, \widetilde{\alpha}_{2}, \ldots, \widetilde{\alpha}_{n}\right)$ be a collection of interval-valued intuitionistic fuzzy arguments, and $\widetilde{\alpha}_{j}=$ $\left(\left[a_{j}, b_{j}\right],\left[c_{j}, d_{j}\right]\right) . \tilde{\mu}=\left(\left[a_{\mu}, b_{\mu}\right],\left[c_{\mu}, d_{\mu}\right]\right)$ denotes mean value of $\left(\widetilde{\alpha}_{1}, \widetilde{\alpha}_{2}, \ldots, \widetilde{\alpha}_{n}\right)$; then the variance of $\widetilde{\alpha}_{1}, \widetilde{\alpha}_{2}, \ldots, \widetilde{\alpha}_{n}$ can be computed according to

$$
\sigma=\sqrt{\frac{1}{n} \sum_{j=1}^{n}\left(d\left(\tilde{\alpha}_{j}, \tilde{\mu}\right)\right)^{2}} .
$$

In real world, a collection of $n$ aggregated arguments $\left(\alpha_{1}, \alpha_{2}, \ldots, \alpha_{n}\right)$ usually takes the form of a collection of $n$ preference values provided by $n$ different decision makers. Some decision makers may assign unduly high or unduly low preference values to their preferred or repugnant objects. In such case, very low weights should be assigned to these "false" or "biased" opinions; that is to say, the closer a preference value argument is to the mid one(s), the more the weight; conversely, the further a preference value is apart from the mid one(s), the less the weight. So, $\mathrm{Xu}$ [44] and $\mathrm{Xu}$ [49] developed Gaussian (normal) distribution-based method to determine OWA weights by utilizing orderings of arguments 
assessed with crisp numbers and interval numbers, respectively. Inspired by these ideas, by using predefined mean value $\tilde{\mu}$ of IVIFNs, we extended the Gaussian distribution method to obtain the dependent weights, here called Gaussian weighting vector, according to interval-valued intuitionistic fuzzy input arguments.

Definition 14. Let $\tilde{\mu}$ be the mean value of given intervalvalued intuitionistic fuzzy arguments, $\sigma$ the variance of given interval-valued intuitionistic fuzzy arguments; then the Gaussian weighting vector $\omega=\left(\omega_{1}, \omega_{2}, \ldots, \omega_{n}\right)^{T}$ can be defined as

$$
\omega_{j}=\frac{1}{\sqrt{2 \pi} \sigma} e^{-d^{2}\left(\widetilde{\beta}_{j}-\tilde{\mu}\right) / 2 \sigma^{2}}, \quad j=1,2, \ldots, n,
$$

where $\left(\widetilde{\beta}_{1}, \widetilde{\beta}_{2}, \ldots, \widetilde{\beta}_{n}\right)$ is a permutation of $\left(\widetilde{\alpha}_{1}, \widetilde{\alpha}_{2}, \ldots, \widetilde{\alpha}_{n}\right)$, with $\widetilde{\beta}_{j-1} \geq \widetilde{\beta}_{j}$ for all $j=2, \ldots, n$.

Consider that $\omega_{j} \in[0,1]$ and $\sum_{j=1}^{n} \omega_{j}=1$ are commonly required in aggregation operators; then we can normalize the Gaussian weighting vector according to

$$
\omega_{j}=\frac{(1 / \sqrt{2 \pi} \sigma) e^{-d^{2}\left(\widetilde{\beta}_{j}-\widetilde{\mu}\right) / 2 \sigma^{2}}}{\sum_{j=1}^{n}(1 / \sqrt{2 \pi} \sigma) e^{-d^{2}\left(\widetilde{\beta}_{j}-\widetilde{\mu}\right) / 2 \sigma^{2}}}, \quad j=1,2, \ldots, n
$$

Then by (17), we can define a Gaussian generalized interval-valued intuitionistic fuzzy ordered weighted averaging (Gaussian-GIIFOWA) operator, as shown in the following definition.

Definition 15. A Gaussian-GIIFOWA operator of dimension $n$ is a mapping Gaussian-GIIFOWA: $\Omega^{n} \rightarrow \Omega$, which has an associated Gaussian weighting vector $\omega=\left(\omega_{1}, \omega_{2}, \ldots, \omega_{n}\right)^{T}$, with $\omega_{i} \in[0,1]$ and $\sum_{i=1}^{n} \omega_{i}=1$; then

$$
\begin{aligned}
& \text { Gaussian-GIIFOWA }\left(\widetilde{\alpha}_{1}, \widetilde{\alpha}_{2}, \ldots, \widetilde{\alpha}_{n}\right) \\
& =\left(\omega_{\tilde{\alpha}_{\sigma(1)}} \widetilde{\alpha}_{\sigma(1)}^{\lambda} \oplus \omega_{\tilde{\alpha}_{\sigma(2)}} \widetilde{\alpha}_{\sigma(2)}^{\lambda} \oplus \cdots \oplus \omega_{\widetilde{\alpha}_{\sigma(n)}} \widetilde{\alpha}_{\sigma(n)}^{\lambda}\right)^{1 / \lambda} \\
& =\left(\frac{(1 / \sqrt{2 \pi} \sigma) e^{-d^{2}\left(\tilde{\beta}_{1}-\widetilde{\mu}\right) / 2 \sigma^{2}}}{\sum_{j=1}^{n}(1 / \sqrt{2 \pi} \sigma) e^{-d^{2}\left(\widetilde{\beta}_{j}-\widetilde{\mu}\right) / 2 \sigma^{2}}} \widetilde{\beta}_{1}^{\lambda}\right. \\
& \oplus \frac{(1 / \sqrt{2 \pi} \sigma) e^{-d^{2}\left(\widetilde{\beta}_{2}-\widetilde{\mu}\right) / 2 \sigma^{2}}}{\sum_{j=1}^{n}(1 / \sqrt{2 \pi} \sigma) e^{-d^{2}\left(\tilde{\beta}_{j}-\widetilde{\mu}\right) / 2 \sigma^{2}}} \widetilde{\beta}_{2}^{\lambda} \\
& \left.\oplus \cdots \oplus \frac{(1 / \sqrt{2 \pi} \sigma) e^{-d^{2}\left(\widetilde{\beta}_{n}-\widetilde{\mu}\right) / 2 \sigma^{2}}}{\sum_{j=1}^{n}(1 / \sqrt{2 \pi} \sigma) e^{-d^{2}\left(\widetilde{\beta}_{j}-\widetilde{\mu}\right) / 2 \sigma^{2}}} \widetilde{\beta}_{n}^{\lambda}\right)^{1 / \lambda} \\
& =\left(\frac{1}{\sqrt{2 \pi} \sigma} e^{-d^{2}\left(\tilde{\beta}_{1}-\widetilde{\mu}\right) / 2 \sigma^{2}} \tilde{\beta}_{1}^{\lambda} \oplus \frac{1}{\sqrt{2 \pi} \sigma} e^{-d^{2}\left(\widetilde{\beta}_{2}-\widetilde{\mu}\right) / 2 \sigma^{2}} \widetilde{\beta}_{2}^{\lambda}\right. \\
& \left.\oplus \cdots \oplus \frac{1}{\sqrt{2 \pi} \sigma} e^{-d^{2}\left(\widetilde{\beta}_{n}-\widetilde{\mu}\right) / 2 \sigma^{2}} \widetilde{\beta}_{n}^{\lambda}\right)^{1 / \lambda} \\
& \times\left(\left(\sum_{j=1}^{n} \frac{1}{\sqrt{2 \pi} \sigma} e^{-d^{2}\left(\widetilde{\beta}_{j}-\widetilde{\mu}\right) / 2 \sigma^{2}}\right)^{1 / \lambda}\right)^{-1},
\end{aligned}
$$

where $\left(\widetilde{\beta}_{1}, \widetilde{\beta}_{2}, \ldots, \widetilde{\beta}_{n}\right)$ is a permutation of $\left(\widetilde{\alpha}_{1}, \widetilde{\alpha}_{2}, \ldots, \widetilde{\alpha}_{n}\right)$, with $\widetilde{\beta}_{j-1} \geq \widetilde{\beta}_{j}$ for all $j=2, \ldots, n$.

Similarly, we can define the Gaussian generalized interval-valued intuitionistic fuzzy ordered weighted geometric (Gaussian-GIIFOWG) operator.

Definition 16. A Gaussian-GIIFOWG operator of dimension $n$ is a mapping Gaussian-GIIFOWG: $\Omega^{n} \rightarrow \Omega$, which has an associated Gaussian weighting vector $\omega=\left(\omega_{1}, \omega_{2}, \ldots, \omega_{n}\right)^{T}$ with $\omega_{i} \in[0,1]$ and $\sum_{i=1}^{n} \omega_{i}=1$; then

$$
\begin{aligned}
& \text { Gaussian-GIIFOWG }\left(\widetilde{\alpha}_{1}, \widetilde{\alpha}_{2}, \ldots, \widetilde{\alpha}_{n}\right)=\frac{1}{\lambda}\left(\left(\lambda \widetilde{\beta}_{1}\right)^{\omega_{\tilde{\beta}(1)}} \otimes\left(\lambda \widetilde{\beta}_{2}\right)^{\omega_{\tilde{\beta}(2)}} \otimes \cdots \otimes\left(\lambda \widetilde{\beta}_{n}\right)^{\omega_{\tilde{\beta}(n)}}\right)
\end{aligned}
$$

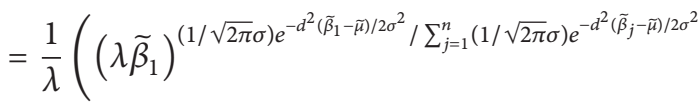

$$
\begin{aligned}
& \otimes\left(\lambda \widetilde{\beta}_{2}\right)^{(1 / \sqrt{2 \pi} \sigma) e^{-d^{2}\left(\widetilde{\beta}_{2}-\widetilde{\mu}\right) / 2 \sigma^{2}} / \sum_{j=1}^{n}(1 / \sqrt{2 \pi} \sigma) e^{-d^{2}\left(\widetilde{\beta}_{j}-\widetilde{\mu}\right) / 2 \sigma^{2}}} \\
& \left.\otimes \cdots \otimes\left(\lambda \widetilde{\beta}_{n}\right)^{(1 / \sqrt{2 \pi} \sigma) e^{-d^{2}\left(\tilde{\beta}_{n}-\tilde{\mu}\right) / 2 \sigma^{2}} / \sum_{j=1}^{n}(1 / \sqrt{2 \pi} \sigma) e^{-d^{2}\left(\tilde{\beta}_{j}-\tilde{\mu}\right) / 2 \sigma^{2}}}\right) \\
& =\frac{1}{\lambda}\left(\left(\lambda \widetilde{\beta}_{1}\right)^{(1 / \sqrt{2 \pi} \sigma) e^{-d^{2}\left(\widetilde{\beta}_{1}-\tilde{\mu}\right) / 2 \sigma^{2}}} \otimes\left(\lambda \widetilde{\beta}_{2}\right)^{(1 / \sqrt{2 \pi} \sigma) e^{-d^{2}\left(\widetilde{\beta}_{2}-\tilde{\mu}\right) / 2 \sigma^{2}}}\right. \\
& \left.\otimes \cdots \otimes\left(\lambda \widetilde{\beta}_{n}\right)^{(1 / \sqrt{2 \pi} \sigma) e^{-d^{2}\left(\tilde{\beta}_{n}-\tilde{\mu}\right) / 2 \sigma^{2}}}\right)^{1 / \sum_{j=1}^{n}(1 / \sqrt{2 \pi} \sigma) e^{-d^{2}\left(\tilde{\beta}_{j}-\tilde{\mu}\right) / 2 \sigma^{2}}},
\end{aligned}
$$


where $\left(\widetilde{\beta}_{1}, \widetilde{\beta}_{2}, \ldots, \widetilde{\beta}_{n}\right)$ is a permutation of $\left(\widetilde{\alpha}_{1}, \widetilde{\alpha}_{2}, \ldots, \widetilde{\alpha}_{n}\right)$ with $\widetilde{\beta}_{j-1} \geq \widetilde{\beta}_{j}$ for all $j=2, \ldots, n$.
Let $\widetilde{\alpha}_{i}=\left(\left[a_{\widetilde{\alpha}(i)}, b_{\widetilde{\alpha}(i)}\right],\left[c_{\widetilde{\alpha}(i)}, d_{\widetilde{\alpha}(i)}\right]\right), \widetilde{\beta}_{i}=\left(\left[a_{\widetilde{\beta}(i)}, b_{\widetilde{\beta}(i)}\right]\right.$, $\left.\left[c_{\widetilde{\beta}(i)}, d_{\tilde{\beta}(i)}\right]\right)$; then by Definition 3, Gaussian-GIIFOWA operator and Gaussian-GIIFOWG operator can be transformed into the following forms;

$\operatorname{Gaussian-GIIFOWA}\left(\widetilde{\alpha}_{1}, \widetilde{\alpha}_{2}, \ldots, \widetilde{\alpha}_{n}\right)=\left(\left[\left(1-\prod_{j=1}^{n}\left(1-a_{\tilde{\beta}(j)}^{\lambda}\right)^{(1 / \sqrt{2 \pi} \sigma) e^{-d^{2}\left(\widetilde{\beta}_{j}-\tilde{\mu}\right) / 2 \sigma^{2}} / \sum_{j=1}^{n}(1 / \sqrt{2 \pi} \sigma) e^{-d^{2}\left(\widetilde{\beta}_{j}-\tilde{\mu}\right) / 2 \sigma^{2}}}\right)^{1 / \lambda}\right.\right.$,

$$
\begin{aligned}
& \left.\left.\left(1-\prod_{j=1}^{n}\left(1-b_{\tilde{\beta}(j)}^{\lambda}\right)\right)^{(1 / \sqrt{2 \pi} \sigma) e^{-d^{2}\left(\tilde{\beta}_{j}-\tilde{\mu}\right) / 2 \sigma^{2}} / \sum_{j=1}^{n}(1 / \sqrt{2 \pi} \sigma) e^{-d^{2}\left(\tilde{\beta}_{j}-\tilde{\mu}\right) / 2 \sigma^{2}}}\right)^{1 / \lambda}\right], \\
& {\left[1-\left(1-\prod_{j=1}^{n}\left(1-\left(1-c_{\widetilde{\beta}(j)}\right)^{\lambda}\right)^{(1 / \sqrt{2 \pi} \sigma) e^{-d^{2}\left(\tilde{\beta}_{j}-\tilde{\mu}\right) / 2 \sigma^{2}} / \sum_{j=1}^{n}(1 / \sqrt{2 \pi} \sigma) e^{-d^{2}\left(\tilde{\beta}_{j}-\tilde{\mu}\right) / 2 \sigma^{2}}}\right)^{1 / \lambda},\right.} \\
& \left.\left.1-\left(1-\prod_{j=1}^{n}\left(1-\left(1-d_{\tilde{\beta}(j)}\right)^{\lambda}\right)^{(1 / \sqrt{2 \pi} \sigma) e^{-d^{2}(\tilde{\beta} j-\tilde{\mu}) / 2 \sigma^{2}} / \sum_{j=1}^{n}(1 / \sqrt{2 \pi} \sigma) e^{-d^{2}\left(\tilde{\beta}_{j}-\tilde{\mu}\right) / 2 \sigma^{2}}}\right)^{1 / \lambda}\right]\right),
\end{aligned}
$$

Gaussian-GIIFOWG $\left(\widetilde{\alpha}_{1}, \widetilde{\alpha}_{2}, \ldots, \widetilde{\alpha}_{n}\right)=\left(\left[1-\left(1-\prod_{j=1}^{n}\left(1-\left(1-a_{\tilde{\beta}(j)}\right)^{\lambda}\right)^{(1 / \sqrt{2 \pi} \sigma) e^{-d^{2}\left(\tilde{\beta}_{j}-\tilde{\mu}\right) / 2 \sigma^{2}} / \sum_{j=1}^{n}(1 / \sqrt{2 \pi} \sigma) e^{-d^{2}\left(\tilde{\beta}_{j}-\tilde{\mu}\right) / 2 \sigma^{2}}}\right)^{1 / \lambda}\right.\right.$,

$$
\begin{aligned}
& \left.1-\left(1-\prod_{j=1}^{n}\left(1-\left(1-b_{\widetilde{\beta}(j)}\right)^{\lambda}\right)^{(1 / \sqrt{2 \pi} \sigma) e^{-d^{2}\left(\tilde{\beta}_{j}-\tilde{\mu}\right) / 2 \sigma^{2}} / \sum_{j=1}^{n}(1 / \sqrt{2 \pi} \sigma) e^{-d^{2}\left(\tilde{\beta}_{j}-\tilde{\mu}\right) / 2 \sigma^{2}}}\right)^{1 / \lambda}\right] \\
& {\left[\left(1-\prod_{j=1}^{n}\left(1-c_{\tilde{\beta}(j)}^{\lambda}\right)^{(1 / \sqrt{2 \pi} \sigma) e^{-d^{2}\left(\tilde{\beta}_{j}-\tilde{\mu}\right) / 2 \sigma^{2}} / \sum_{j=1}^{n}(1 / \sqrt{2 \pi} \sigma) e^{-d^{2}\left(\tilde{\beta}_{j}-\tilde{\mu}\right) / 2 \sigma^{2}}}\right)^{1 / \lambda},\right.} \\
& \left.\left.\left(1-\prod_{j=1}^{n}\left(1-d_{\tilde{\beta}(j)}^{\lambda}\right)^{(1 / \sqrt{2 \pi} \sigma) e^{-d^{2}\left(\tilde{\beta}_{j}-\tilde{\mu}\right) / 2 \sigma^{2}} / \sum_{j=1}^{n}(1 / \sqrt{2 \pi} \sigma) e^{-d^{2}\left(\tilde{\beta}_{j}-\tilde{\mu}\right) / 2 \sigma^{2}}}\right)^{1 / \lambda}\right]\right), \\
& \frac{1}{\sqrt{2 \pi} \sigma} e^{-d^{2}\left(\widetilde{\beta}_{1}-\widetilde{\mu}\right) / 2 \sigma^{2}} \widetilde{\beta}_{1}^{\lambda} \oplus \frac{1}{\sqrt{2 \pi} \sigma} e^{-d^{2}\left(\widetilde{\beta}_{2}-\widetilde{\mu}\right) / 2 \sigma^{2}} \widetilde{\beta}_{2}^{\lambda} \oplus \cdots \oplus \frac{1}{\sqrt{2 \pi} \sigma} e^{-d^{2}\left(\widetilde{\beta}_{n}-\widetilde{\mu}\right) / 2 \sigma^{2}} \widetilde{\beta}_{n}^{\lambda} \\
& =\frac{1}{\sqrt{2 \pi} \sigma} e^{-d^{2}\left(\widetilde{\alpha}_{1}-\tilde{\mu}\right) / 2 \sigma^{2}} \widetilde{\alpha}_{1}^{\lambda} \oplus \frac{1}{\sqrt{2 \pi} \sigma} e^{-d^{2}\left(\widetilde{\alpha}_{2}-\tilde{\mu}\right) / 2 \sigma^{2}} \widetilde{\alpha}_{2}^{\lambda} \\
& \oplus \cdots \oplus \frac{1}{\sqrt{2 \pi} \sigma} e^{-d^{2}\left(\widetilde{\alpha}_{n}-\widetilde{\mu}\right) / 2 \sigma^{2}} \widetilde{\alpha}_{n}^{\lambda} \\
& \left(\sum_{j=1}^{n} \frac{1}{\sqrt{2 \pi} \sigma} e^{-d^{2}\left(\widetilde{\beta}_{j}-\widetilde{\mu}\right) / 2 \sigma^{2}}\right)^{1 / \lambda}=\left(\sum_{j=1}^{n} \frac{1}{\sqrt{2 \pi} \sigma} e^{-d^{2}\left(\widetilde{\alpha}_{j}-\widetilde{\mu}\right) / 2 \sigma^{2}}\right)^{1 / \lambda}, \\
& \left(\left(\lambda \widetilde{\beta}_{1}\right)^{(1 / \sqrt{2 \pi} \sigma) e^{-d^{2}\left(\tilde{\beta}_{1}-\tilde{\mu}\right) / 2 \sigma^{2}}} \otimes\left(\lambda \widetilde{\beta}_{2}\right)^{(1 / \sqrt{2 \pi} \sigma) e^{-d^{2}\left(\tilde{\beta}_{2}-\tilde{\mu}\right) / 2 \sigma^{2}}}\right. \\
& \left.\otimes \cdots \otimes\left(\lambda \widetilde{\beta}_{n}\right)^{(1 / \sqrt{2 \pi} \sigma) e^{-d^{2}\left(\tilde{\beta}_{n}-\tilde{\mu}\right) / 2 \sigma^{2}}}\right)^{1 / \sum_{j=1}^{n}(1 / \sqrt{2 \pi} \sigma) e^{-d^{2}\left(\widetilde{\beta}_{j}-\tilde{\mu}\right) / 2 \sigma^{2}}} \\
& =\left(\left(\lambda \widetilde{\alpha}_{1}\right)^{(1 / \sqrt{2 \pi} \sigma) e^{-d^{2}\left(\tilde{\alpha}_{1}-\tilde{\mu}\right) / 2 \sigma^{2}}} \otimes\left(\lambda \widetilde{\alpha}_{2}\right)^{(1 / \sqrt{2 \pi} \sigma) e^{-d^{2}\left(\tilde{\alpha}_{2}-\tilde{\mu}\right) / 2 \sigma^{2}}},\right. \\
& \left.\otimes \cdots \otimes\left(\lambda \widetilde{\alpha}_{n}\right)^{(1 / \sqrt{2 \pi} \sigma) e^{-d^{2}\left(\tilde{\alpha}_{n}-\tilde{\mu}\right) / 2 \sigma^{2}}}\right)^{1 / \sum_{j=1}^{n}(1 / \sqrt{2 \pi} \sigma) e^{-d^{2}\left(\tilde{\alpha}_{j}-\tilde{\mu}\right) / 2 \sigma^{2}}}
\end{aligned}
$$


then we can rewrite as

$$
\begin{aligned}
& \text { Gaussian-GIIFOWA }\left(\widetilde{\alpha}_{1}, \widetilde{\alpha}_{2}, \ldots, \widetilde{\alpha}_{n}\right) \\
& =\left(\omega_{\widetilde{\alpha}(1)} \widetilde{\alpha}_{1}^{\lambda} \oplus \omega_{\widetilde{\alpha}(2)} \widetilde{\alpha}_{2}^{\lambda} \oplus \cdots \oplus \omega_{\widetilde{\alpha}(n)} \widetilde{\alpha}_{n}^{\lambda}\right)^{1 / \lambda},
\end{aligned}
$$

Gaussian-GIIFOWG $\left(\widetilde{\alpha}_{1}, \widetilde{\alpha}_{2}, \ldots, \widetilde{\alpha}_{n}\right)$

$$
=\frac{1}{\lambda}\left(\left(\lambda \widetilde{\alpha}_{1}\right)^{\omega_{\widetilde{\alpha}(1)}} \otimes\left(\lambda \widetilde{\alpha}_{2}\right)^{\omega_{\widetilde{\alpha}(2)}} \otimes \cdots \otimes\left(\lambda \widetilde{\alpha}_{n}\right)^{\omega_{\widetilde{\alpha}(n)}}\right) .
$$

Obviously, the aggregated results of Gaussian-GIIFOWA operator and Gaussian-GIIFOWG operator are independent of orderings; thus, Gaussian-GIIFOWA and GaussianGIIFOWG are neat and dependent operators.

Theorem 17. Let $\tilde{\alpha}_{j}=\left(\left[a_{j}, b_{j}\right],\left[c_{j}, d_{j}\right]\right)(j=1,2, \ldots, n)$ be a collection of interval-valued intuitionistic fuzzy arguments, and the $\omega=\left(\omega_{1}, \omega_{2}, \ldots, \omega_{n}\right)^{T}$ be the Gaussian weighting vector related to Gaussian-GIIFOWA operator and GaussianGIIFOWG operator, with $\omega_{j} \in[0,1]$ and $\sum_{j=1}^{n} \omega_{j}=1$; then Gaussian-GIIFOWA operator and Gaussian-GIIFOWG operator have the following properties.

(1) Commutativity: let $\left(\widetilde{\alpha}_{1}^{*}, \widetilde{\alpha}_{2}^{*}, \ldots, \widetilde{\alpha}_{n}^{*}\right)$ be any a permutation of $\left(\widetilde{\alpha}_{1}, \widetilde{\alpha}_{2}, \ldots, \widetilde{\alpha}_{n}\right)$; then

$$
\begin{aligned}
& \text { Gaussian-GIIFOWA }{ }_{\omega, \lambda}\left(\widetilde{\alpha}_{1}^{*}, \widetilde{\alpha}_{2}^{*}, \ldots, \widetilde{\alpha}_{n}^{*}\right) \\
& =\text { Gaussian-GIIFOWA }{ }_{\omega, \lambda}\left(\widetilde{\alpha}_{1}, \widetilde{\alpha}_{2}, \ldots, \widetilde{\alpha}_{n}\right), \\
& \text { Gaussian-GIIFOWG }{ }_{\omega, \lambda}\left(\widetilde{\alpha}_{1}^{*}, \widetilde{\alpha}_{2}^{*}, \ldots, \widetilde{\alpha}_{n}^{*}\right) \\
& =\text { Gaussian-GIIFOWG }{ }_{\omega, \lambda}\left(\widetilde{\alpha}_{1}, \widetilde{\alpha}_{2}, \ldots, \widetilde{\alpha}_{n}\right) .
\end{aligned}
$$

(2) Idempotency: let $\widetilde{\alpha}_{j}=\tilde{\alpha}$, for all $j=1,2, \ldots, n$; then

$$
\begin{aligned}
& \text { Gaussian-GIIFOWA } A_{\omega, \lambda}\left(\widetilde{\alpha}_{1}, \widetilde{\alpha}_{2}, \ldots, \widetilde{\alpha}_{n}\right)=\widetilde{\alpha} \\
& \text { Gaussian-GIIFOWG }{ }_{\omega, \lambda}\left(\widetilde{\alpha}_{1}, \widetilde{\alpha}_{2}, \ldots, \widetilde{\alpha}_{n}\right)=\widetilde{\alpha},
\end{aligned}
$$

(3) Boundedness: the Gaussian-GIIFOWA operator and the Gaussian-GIIFOWG operator lie between the max and min operators;

$$
\begin{aligned}
& \widetilde{\alpha}^{-} \leq \text {Gaussian-GIIFOWA }{ }_{\omega, \lambda}\left(\widetilde{\alpha}_{1}, \widetilde{\alpha}_{2}, \ldots, \widetilde{\alpha}_{n}\right) \leq \widetilde{\alpha}^{+}, \\
& \widetilde{\alpha}^{-} \leq \text {Gaussian-GIIFOWG }{ }_{\omega, \lambda}\left(\widetilde{\alpha}_{1}, \widetilde{\alpha}_{2}, \ldots, \widetilde{\alpha}_{n}\right) \leq \widetilde{\alpha}^{+},
\end{aligned}
$$

where

$$
\begin{aligned}
& \tilde{\alpha}^{-}=\left(\left[\min _{j}\left(a_{j}\right), \min _{j}\left(b_{j}\right)\right],\left[\max _{j}\left(c_{j}\right), \max _{j}\left(d_{j}\right)\right]\right), \\
& \tilde{\alpha}^{+}=\left(\left[\max _{j}\left(a_{j}\right), \max _{j}\left(b_{j}\right)\right],\left[\min _{j}\left(c_{j}\right), \min _{j}\left(d_{j}\right)\right]\right) .
\end{aligned}
$$

Theorem 18. Let $\widetilde{\alpha}_{j}=\left(\left[a_{\widetilde{\alpha}(j)}, b_{\widetilde{\alpha}(j)}\right],\left[c_{\widetilde{\alpha}(j)}, d_{\widetilde{\alpha}(j)}\right]\right), \widetilde{\beta}_{j}=$ $\left(\left[a_{\widetilde{\beta}(j)}, b_{\widetilde{\beta}(j)}\right],\left[c_{\widetilde{\beta}(j)}, d_{\widetilde{\beta}(j)}\right]\right)$ be two collections of interval-valued intuitionistic fuzzy arguments, $\left(\widetilde{\beta}_{1}, \widetilde{\beta}_{2}, \ldots, \widetilde{\beta}_{n}\right)$ a permutation of $\left(\widetilde{\alpha}_{1}, \widetilde{\alpha}_{2}, \ldots, \widetilde{\alpha}_{n}\right)$ with $\widetilde{\beta}_{j-1} \geq \widetilde{\beta}_{j}$ for all $j=2, \ldots, n$, and $\omega=\left(\omega_{1}, \omega_{2}, \ldots, \omega_{n}\right)^{T}$ the Gaussian weighting vector related to the Gaussian-GIIFOWA operator and Gaussian-GIIFOWG operator, with $\omega_{j} \in[0,1]$ and $\sum_{j=1}^{n} \omega_{j}=1$; then

(1) if $\lambda=1$, then the Gaussian-GIIFOWA operator and Gaussian-GIIFOWG operator reduce to the following Gaussian-IIFOWA operator and Gaussian-IIFOWG operator:

$$
\begin{aligned}
& \text { Gaussian-IIFOWA }\left(\widetilde{\alpha}_{1}, \widetilde{\alpha}_{2}, \ldots, \widetilde{\alpha}_{n}\right)= \frac{(1 / \sqrt{2 \pi} \sigma) e^{-d^{2}\left(\widetilde{\beta}_{1}-\widetilde{\mu}\right) / 2 \sigma^{2}}}{\sum_{j=1}^{n}(1 / \sqrt{2 \pi} \sigma) e^{-d^{2}\left(\widetilde{\beta}_{j}-\widetilde{\mu}\right) / 2 \sigma^{2}}} \widetilde{\beta}_{1} \\
& \oplus \frac{(1 / \sqrt{2 \pi} \sigma) e^{-d^{2}\left(\widetilde{\beta}_{2}-\widetilde{\mu}\right) / 2 \sigma^{2}}}{\sum_{j=1}^{n}(1 / \sqrt{2 \pi} \sigma) e^{-d^{2}\left(\widetilde{\beta}_{j}-\widetilde{\mu}\right) / 2 \sigma^{2}}} \widetilde{\beta}_{2} \\
& \oplus \cdots \oplus \frac{(1 / \sqrt{2 \pi} \sigma) e^{-d^{2}\left(\widetilde{\beta}_{n}-\widetilde{\mu}\right) / 2 \sigma^{2}}}{\sum_{j=1}^{n}(1 / \sqrt{2 \pi} \sigma) e^{-d^{2}\left(\widetilde{\beta}_{j}-\widetilde{\mu}\right) / 2 \sigma^{2}} \widetilde{\beta}_{n}} \\
&= \frac{1}{\sqrt{2 \pi} \sigma} e^{-d^{2}\left(\widetilde{\beta_{1}}-\widetilde{\mu}\right) / 2 \sigma^{2} \widetilde{\beta}_{1} \oplus \frac{1}{\sqrt{2 \pi} \sigma} e^{-d^{2}\left(\widetilde{\beta}_{2}-\widetilde{\mu}\right) / 2 \sigma^{2}} \widetilde{\beta}_{2}} \\
& \oplus \cdots \oplus \frac{1}{\sqrt{2 \pi} \sigma} e^{-d^{2}\left(\tilde{\beta}_{n}-\widetilde{\mu}\right) / 2 \sigma^{2}} \widetilde{\beta}_{n} \\
& \times\left(\sum_{j=1}^{n} \frac{1}{\sqrt{2 \pi} \sigma} e^{-d^{2}\left(\widetilde{\beta}_{j}-\widetilde{\mu}\right) / 2 \sigma^{2}}\right)^{-1}
\end{aligned}
$$




$$
\begin{aligned}
& =\left(\left[1-\prod_{j=1}^{n}\left(1-a_{\widetilde{\beta}(j)}\right)^{(1 / \sqrt{2 \pi} \sigma) e^{-d^{2}\left(\widetilde{\beta}_{j}-\widetilde{\mu}\right) / 2 \sigma^{2}} / \sum_{j=1}^{n}(1 / \sqrt{2 \pi} \sigma) e^{-d^{2}\left(\widetilde{\beta}_{j}-\tilde{\mu}\right) / 2 \sigma^{2}}},\right.\right. \\
& \left.1-\prod_{j=1}^{n}\left(1-b_{\widetilde{\beta}(j)}\right)^{(1 / \sqrt{2 \pi} \sigma) e^{-d^{2}\left(\tilde{\beta}_{j}-\widetilde{\mu}\right) / 2 \sigma^{2}} / \sum_{j=1}^{n}(1 / \sqrt{2 \pi} \sigma) e^{-d^{2}\left(\widetilde{\beta}_{j}-\tilde{\mu}\right) / 2 \sigma^{2}}}\right],
\end{aligned}
$$

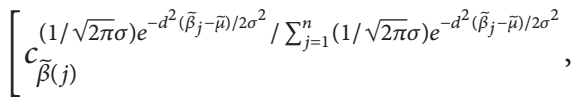

$$
\begin{aligned}
& \left.\left.d_{\widetilde{\beta}(j)}^{(1 / \sqrt{2 \pi} \sigma) e^{-d^{2}\left(\widetilde{\beta}_{j}-\tilde{\mu}\right) / 2 \sigma^{2}} / \sum_{j=1}^{n}(1 / \sqrt{2 \pi} \sigma) e^{-d^{2}\left(\widetilde{\beta}_{j}-\widetilde{\mu}\right) / 2 \sigma^{2}}}\right]\right),
\end{aligned}
$$

Gaussian-IIFOWG $\left(\widetilde{\alpha}_{1}, \widetilde{\alpha}_{2}, \ldots, \widetilde{\alpha}_{n}\right)=\widetilde{\beta}_{1}^{(1 / \sqrt{2 \pi} \sigma) e^{-d^{2}\left(\widetilde{\beta}_{1}-\tilde{\mu}\right) / 2 \sigma^{2}} / \sum_{j=1}^{n}(1 / \sqrt{2 \pi} \sigma) e^{-d^{2}\left(\widetilde{\beta}_{j}-\tilde{\mu}\right) / 2 \sigma^{2}}}$

$$
\begin{aligned}
& \otimes \widetilde{\beta}_{2}^{(1 / \sqrt{2 \pi} \sigma) e^{-d^{2}\left(\widetilde{\beta}_{2}-\tilde{\mu}\right) / 2 \sigma^{2}} / \sum_{j=1}^{n}(1 / \sqrt{2 \pi} \sigma) e^{-d^{2}\left(\tilde{\beta}_{j}-\tilde{\mu}\right) / 2 \sigma^{2}}}
\end{aligned}
$$

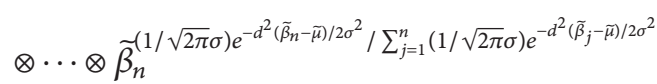

$$
\begin{aligned}
& =\left(\widetilde{\beta}_{1}^{(1 / \sqrt{2 \pi} \sigma) e^{-d^{2}\left(\widetilde{\beta}_{1}-\tilde{\mu}\right) / 2 \sigma^{2}}} \otimes \widetilde{\beta}_{2}^{(1 / \sqrt{2 \pi} \sigma) e^{-d^{2}\left(\widetilde{\beta}_{2}-\tilde{\mu}\right) / 2 \sigma^{2}}}\right. \\
& \left.\otimes \cdots \otimes \widetilde{\beta}_{n}^{(1 / \sqrt{2 \pi} \sigma) e^{-d^{2}\left(\tilde{\beta}_{n}-\widetilde{\mu}\right) / 2 \sigma^{2}}}\right)^{1 / \sum_{j=1}^{n}(1 / \sqrt{2 \pi} \sigma) e^{-d^{2}\left(\tilde{\beta}_{j}-\tilde{\mu}\right) / 2 \sigma^{2}}} \\
& =\left(\left[\prod_{j=1}^{n} a_{\tilde{\beta}(j)}^{(1 / \sqrt{2 \pi} \sigma) e^{-d^{2}\left(\tilde{\beta}_{j}-\tilde{\mu}\right) / 2 \sigma^{2}} / \sum_{j=1}^{n}(1 / \sqrt{2 \pi} \sigma) e^{-d^{2}\left(\tilde{\beta}_{j}-\tilde{\mu}\right) / 2 \sigma^{2}}},\right.\right.
\end{aligned}
$$

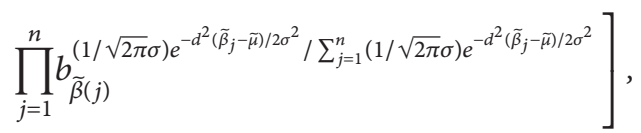

$$
\begin{aligned}
& {\left[1-\prod_{j=1}^{n}\left(1-c_{\widetilde{\beta}(j)}\right)^{(1 / \sqrt{2 \pi} \sigma) e^{-d^{2}\left(\widetilde{\beta}_{j}-\tilde{\mu}\right) / 2 \sigma^{2}} / \sum_{j=1}^{n}(1 / \sqrt{2 \pi} \sigma) e^{-d^{2}\left(\widetilde{\beta}_{j}-\tilde{\mu}\right) / 2 \sigma^{2}}},\right.} \\
& \left.\left.1-\prod_{j=1}^{n}\left(1-d_{\widetilde{\beta}(j)}\right)^{(1 / \sqrt{2 \pi} \sigma) e^{-d^{2}\left(\tilde{\beta}_{j}-\tilde{\mu}\right) / 2 \sigma^{2}} / \sum_{j=1}^{n}(1 / \sqrt{2 \pi} \sigma) e^{-d^{2}\left(\tilde{\beta}_{j}-\tilde{\mu}\right) / 2 \sigma^{2}}}\right]\right)
\end{aligned}
$$

(2) if $\lambda \rightarrow 0$, then the Gaussian-GIIFOWA operator reduces to the Gaussian-IIFOWG operator;
(3) if $\omega=(1 / n, 1 / n, \ldots, 1 / n)^{T}$, then the GaussianGIIFOWA operator and Gaussian-GIIFOWG 
operator reduce to the GIIFA operator and GIIFG operator;

(4) if $\omega=(1 / n, 1 / n, \ldots, 1 / n)^{T}$ and $\lambda=1$, then the Gaussian-GIIFOWA operator and GaussianGIIFOWG operator reduce to the IIFA operator and IIFG operator;

(5) if $\omega=(1 / n, 1 / n, \ldots, 1 / n)^{T}$ and $\lambda \rightarrow 0$, then the Gaussian-GIIFOWA operator reduces to the IIFG operator.

Lemma 19. Assume that $x_{j}>0, \lambda_{j}>0, j=1,2, \ldots, n$, and $\sum_{j=1}^{n} \lambda_{j}=1$, then

$$
\prod_{j=1}^{n} x_{j}^{\lambda_{j}} \leq \sum_{j=1}^{n} \lambda_{j} x_{j},
$$

with equality if and only if $x_{1}=x_{2}=\cdots=x_{n}$.
Theorem 20. Let $\widetilde{\alpha}_{j}=\left(\left[a_{\widetilde{\alpha}(j)}, b_{\widetilde{\alpha}(j)}\right],\left[c_{\widetilde{\alpha}(j)}, d_{\widetilde{\alpha}(j)}\right]\right), \widetilde{\beta}_{j}=$ $\left(\left[a_{\widetilde{\beta}(j)}, b_{\widetilde{\beta}(j)}\right],\left[c_{\widetilde{\beta}(j)}, d_{\widetilde{\beta}(j)}\right]\right)$ be two collections of interval-valued intuitionistic fuzzy arguments, $\left(\widetilde{\beta}_{1}, \widetilde{\beta}_{2}, \ldots, \widetilde{\beta}_{n}\right)$ is a permutation of $\left(\widetilde{\alpha}_{1}, \widetilde{\alpha}_{2}, \ldots, \widetilde{\alpha}_{n}\right)$ with $\widetilde{\beta}_{j-1} \geq \widetilde{\beta}_{j}$ for all $j=2, \ldots, n$, and let $\omega=\left(\omega_{1}, \omega_{2}, \ldots, \omega_{n}\right)^{T}$ be the Gaussian weighting vector related to the Gaussian-GIIFOWA operator and GaussianGIIFOWG operator, with $\omega_{j} \in[0,1]$ and $\sum_{j=1}^{n} \omega_{j}=1$, then

(1) Gaussian-IIFOWG $\left(\widetilde{\alpha}_{1}, \widetilde{\alpha}_{2}, \ldots, \widetilde{\alpha}_{n}\right) \leq$ Gaussian$\operatorname{IIFOWA}\left(\widetilde{\alpha}_{1}, \widetilde{\alpha}_{2}, \ldots, \tilde{\alpha}_{n}\right)$;

(2) Gaussian-IIFOWG $\left(\widetilde{\alpha}_{1}, \widetilde{\alpha}_{2}, \ldots, \widetilde{\alpha}_{n}\right) \leq$ Gaussian$\operatorname{GIIFOWA} A_{\lambda}\left(\widetilde{\alpha}_{1}, \widetilde{\alpha}_{2}, \ldots, \widetilde{\alpha}_{n}\right) ;$

(3) Gaussian-GIIFOWG ${ }_{\lambda}\left(\widetilde{\alpha}_{1}, \widetilde{\alpha}_{2}, \ldots, \widetilde{\alpha}_{n}\right) \leq$ Gaussian$\operatorname{IIFOWA}\left(\widetilde{\alpha}_{1}, \widetilde{\alpha}_{2}, \ldots, \widetilde{\alpha}_{n}\right)$.

Proof. Based on Lemma 19, we can have

$$
\begin{aligned}
& \prod_{j=1}^{n} a_{\widetilde{\beta}(j)}^{(1 / \sqrt{2 \pi} \sigma) e^{-d^{2}\left(\tilde{\beta}_{j}-\tilde{\mu}\right) / 2 \sigma^{2}} / \sum_{j=1}^{n}(1 / \sqrt{2 \pi} \sigma) e^{-d^{2}\left(\tilde{\beta}_{j}-\tilde{\mu}\right) / 2 \sigma^{2}}} \\
& \leq \sum_{j=1}^{n} \frac{(1 / \sqrt{2 \pi} \sigma) e^{-d^{2}\left(\widetilde{\beta}_{j}-\widetilde{\mu}\right) / 2 \sigma^{2}}}{\sum_{j=1}^{n}(1 / \sqrt{2 \pi} \sigma) e^{-d^{2}\left(\widetilde{\beta}_{j}-\widetilde{\mu}\right) / 2 \sigma^{2}}} a_{\widetilde{\beta}(j)} \\
& =1-\sum_{j=1}^{n} \frac{(1 / \sqrt{2 \pi} \sigma) e^{-d^{2}\left(\widetilde{\beta}_{j}-\widetilde{\mu}\right) / 2 \sigma^{2}}}{\sum_{j=1}^{n}(1 / \sqrt{2 \pi} \sigma) e^{-d^{2}\left(\widetilde{\beta}_{j}-\widetilde{\mu}\right) / 2 \sigma^{2}}}\left(1-a_{\widetilde{\beta}(j)}\right)
\end{aligned}
$$

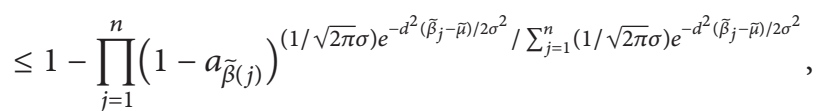

$$
\begin{aligned}
& \prod_{j=1}^{n} a_{\widetilde{\beta}(j)}^{(1 / \sqrt{2 \pi} \sigma) e^{-d^{2}\left(\tilde{\beta}_{j}-\tilde{\mu}\right) / 2 \sigma^{2}} / \sum_{j=1}^{n}(1 / \sqrt{2 \pi} \sigma) e^{-d^{2}\left(\tilde{\beta}_{j}-\tilde{\mu}\right) / 2 \sigma^{2}}} \\
& =\left(\prod_{j=1}^{n}\left(a_{\widetilde{\beta}(j)}^{\lambda}\right)^{(1 / \sqrt{2 \pi} \sigma) e^{-d^{2}\left(\tilde{\beta}_{j}-\tilde{\mu}\right) / 2 \sigma^{2}} / \sum_{j=1}^{n}(1 / \sqrt{2 \pi} \sigma) e^{-d^{2}\left(\tilde{\beta}_{j}-\tilde{\mu}\right) / 2 \sigma^{2}}}\right)^{1 / \lambda} \\
& \leq\left(\sum_{j=1}^{n} \frac{(1 / \sqrt{2 \pi} \sigma) e^{-d^{2}\left(\tilde{\beta}_{j}-\widetilde{\mu}\right) / 2 \sigma^{2}}}{\sum_{j=1}^{n}(1 / \sqrt{2 \pi} \sigma) e^{-d^{2}\left(\tilde{\beta}_{j}-\tilde{\mu}\right) / 2 \sigma^{2}}} a_{\widetilde{\beta}(j)}^{\lambda}\right)^{1 / \lambda} \\
& =\left(1-\sum_{j=1}^{n} \frac{(1 / \sqrt{2 \pi} \sigma) e^{-d^{2}\left(\widetilde{\beta}_{j}-\widetilde{\mu}\right) / 2 \sigma^{2}}}{\sum_{j=1}^{n}(1 / \sqrt{2 \pi} \sigma) e^{-d^{2}\left(\tilde{\beta}_{j}-\widetilde{\mu}\right) / 2 \sigma^{2}}}\left(1-a_{\widetilde{\beta}(j)}^{\lambda}\right)\right)^{1 / \lambda} \\
& \leq\left(1-\prod_{j=1}^{n}\left(1-a_{\widetilde{\beta}(j)}^{\lambda}\right)^{(1 / \sqrt{2 \pi} \sigma) e^{-d^{2}\left(\widetilde{\beta}_{j}-\tilde{\mu}\right) / 2 \sigma^{2}} / \sum_{j=1}^{n}(1 / \sqrt{2 \pi} \sigma) e^{-d^{2}\left(\tilde{\beta}_{j}-\tilde{\mu}\right) / 2 \sigma^{2}}}\right)^{1 / \lambda},
\end{aligned}
$$




$$
\begin{aligned}
& 1-\left(1-\prod_{j=1}^{n}\left(1-\left(1-a_{\widetilde{\beta}(j)}\right)^{\lambda}\right)^{(1 / \sqrt{2 \pi} \sigma) e^{-d^{2}\left(\widetilde{\beta}_{j}-\widetilde{\mu}\right) / 2 \sigma^{2}} / \sum_{j=1}^{n}(1 / \sqrt{2 \pi} \sigma) e^{-d^{2}\left(\widetilde{\beta}_{j}-\tilde{\mu}\right) / 2 \sigma^{2}}}\right)^{1 / \lambda} \\
& \leq 1-\left(1-\sum_{j=1}^{n} \frac{(1 / \sqrt{2 \pi} \sigma) e^{-d^{2}\left(\widetilde{\beta}_{j}-\widetilde{\mu}\right) / 2 \sigma^{2}}}{\sum_{j=1}^{n}(1 / \sqrt{2 \pi} \sigma) e^{-d^{2}\left(\tilde{\beta}_{j}-\tilde{\mu}\right) / 2 \sigma^{2}}}\left(1-\left(1-a_{\widetilde{\beta}(j)}\right)^{\lambda}\right)\right)^{1 / \lambda} \\
& =1-\left(\sum_{j=1}^{n} \frac{(1 / \sqrt{2 \pi} \sigma) e^{-d^{2}\left(\tilde{\beta}_{j}-\widetilde{\mu}\right) / 2 \sigma^{2}}}{\sum_{j=1}^{n}(1 / \sqrt{2 \pi} \sigma) e^{-d^{2}\left(\tilde{\beta}_{j}-\tilde{\mu}\right) / 2 \sigma^{2}}}\left(1-a_{\tilde{\beta}(j)}\right)^{\lambda}\right)^{1 / \lambda} \\
& \leq 1-\left(\prod_{j=1}^{n}\left(1-a_{\tilde{\beta}(j)}\right)^{\lambda(1 / \sqrt{2 \pi} \sigma) e^{-d^{2}\left(\tilde{\beta}_{j}-\tilde{\mu}\right) / 2 \sigma^{2}} / \sum_{j=1}^{n}(1 / \sqrt{2 \pi} \sigma) e^{-d^{2}\left(\widetilde{\beta}_{j}-\tilde{\mu}\right) / 2 \sigma^{2}}}\right)^{1 / \lambda} \\
& =1-\prod_{j=1}^{n}\left(1-a_{\widetilde{\beta}(j)}\right)^{(1 / \sqrt{2 \pi} \sigma) e^{-d^{2}\left(\widetilde{\beta}_{j}-\tilde{\mu}\right) / 2 \sigma^{2}} / \sum_{j=1}^{n}(1 / \sqrt{2 \pi} \sigma) e^{-d^{2}\left(\widetilde{\beta}_{j}-\tilde{\mu}\right) / 2 \sigma^{2}}} .
\end{aligned}
$$

Obviously, the above inequations (a), (b), and (c) are also valid for $b_{\widetilde{\beta}(j)}, c_{\widetilde{\beta}(j)}$, and $d_{\widetilde{\beta}(j)}$.

Then by Lemma 19, we can have

$$
\begin{aligned}
& {\underset{j=1}{\otimes}}_{j}^{n}\left(\widetilde{\beta}_{j}^{\omega_{j}}\right) \leq \bigoplus_{j=1}^{n}\left(\omega_{j} \widetilde{\beta}_{j}\right), \\
& \bigotimes_{j=1}^{n}\left(\widetilde{\beta}_{j}^{\omega_{j}}\right) \leq\left(\bigoplus_{j=1}^{n}\left(\omega_{j} \widetilde{\beta}_{j}^{\lambda}\right)\right)^{1 / \lambda} \\
& \frac{1}{\lambda}\left(\bigotimes_{j=1}^{n}\left(\lambda \widetilde{\beta}_{j}\right)^{w_{j}}\right) \leq \bigoplus_{j=1}^{n}\left(\omega_{j} \widetilde{\beta}_{j}\right)
\end{aligned}
$$

and thus complete the proof of Theorem 20.

Example 21. For a group decision making problem, suppose that there are six decision makers $d_{j}(j=1,2, \ldots, 6)$; these decision makers provide their individual preferences with interval-valued intuitionistic fuzzy numbers. Then, the preference arguments are collected as follows:

$$
\begin{aligned}
& \tilde{\alpha}_{1}=([0.5,0.6],[0.2,0.4]), \\
& \widetilde{\alpha}_{2}=([0.3,0.6],[0.3,0.4]), \\
& \widetilde{\alpha}_{3}=([0.4,0.7],[0.2,0.3]), \\
& \widetilde{\alpha}_{4}=([0.3,0.5],[0.1,0.4]), \\
& \widetilde{\alpha}_{5}=([0.4,0.7],[0.2,0.3]) \\
& \tilde{\alpha}_{6}=([0.6,0.8],[0.0,0.2]) .
\end{aligned}
$$

Utilizing (21) and (22), the mean value $\tilde{u}$ and variance value $\sigma$ can be obtained:

$$
\widetilde{u}=([0.4273,0.664],[0,0.3238]), \quad \sigma=0.1271 .
$$

Then, by (23) and (24), we can compute the Gaussian weighting vector:

$$
\omega=\left(\omega_{1}, \omega_{2}, \ldots, \omega_{6}\right),
$$

where $\omega_{1}=0.1391, \omega_{2}=0.128, \omega_{3}=0.1867, \omega_{4}=0.192$, $\omega_{5}=0.1867$, and $\omega_{6}=0.1675$.

Given $\lambda=5$, according to (27) and (28), it follows that

$$
\begin{aligned}
& \text { Gaussian-GIIFOWA }\left(\widetilde{\alpha}_{1}, \widetilde{\alpha}_{2}, \ldots, \widetilde{\alpha}_{n}\right) \\
& =([0.4676,0.6846],[0.0,0.3083]), \\
& \text { Gaussian-GIIFOWG }\left(\widetilde{\alpha}_{1}, \widetilde{\alpha}_{2}, \ldots, \widetilde{\alpha}_{n}\right) \\
& =([0.381,0.6038],[0.2166,0.3554]) .
\end{aligned}
$$

3.3. Proposed Power Generalized Interval-Valued Intuitionistic Fuzzy Aggregation Operators. The above-presented Gaussian distribution-based methods can obtain argument-dependent weights according to the indirectly calculated support degree of arguments by considering the distances between arguments and the mid one (mean value). On the other hand, to directly consider the support degree of each argument, Yager [54] developed the power average (PA) operator and a power ordered weighted average (POWA) operator, which allow the arguments being aggregated to support each other. Then, $\mathrm{Xu}$ and Yager [39] developed power geometric average (PGA) operator and power ordered weighted average (POWA) operator. Most recently, Zhou and Chen [9] further studied extensions of power operator to linguistic decision environment. Motivated by these ideas, here we first devise a hybrid support function for interval-valued intuitionistic fuzzy input arguments to not only consider the support degrees of each argument by other arguments but also consider the support degrees between argument values and mid one (mean value). Then, a power generalized interval-valued intuitionistic fuzzy ordered weighted averaging (P-GIIFOWA) operator and a power generalized interval-valued intuitionistic fuzzy ordered weighted geometric (P-GIIFOWG) operator are defined, in which associated weights are obtained by the devised hybrid support function. 
Definition 22. Let $\left(\widetilde{\alpha}_{1}, \widetilde{\alpha}_{2}, \ldots, \widetilde{\alpha}_{n}\right)$ be a collection of intervalvalued intuitionistic fuzzy arguments, and let $\tilde{\mu}$ denote the mean value; then the hybrid support function can be defined as

$$
\begin{aligned}
\operatorname{Sup}\left(\tilde{\alpha}_{j}\right) & =\frac{1}{n-1} \sum_{k=1, j \neq k}^{n}\left(1-d\left(\tilde{\alpha}_{j}, \widetilde{\alpha}_{k}\right)\right)+\left(1-d\left(\tilde{\alpha}_{j}, \tilde{\mu}\right)\right) \\
& =\frac{1}{n-1} \sum_{k=1, j \neq k}^{n} \operatorname{Sup}\left(\widetilde{\alpha}_{j}, \widetilde{\alpha}_{k}\right)+\operatorname{Sup}\left(\widetilde{\alpha}_{j}, \tilde{\mu}\right) .
\end{aligned}
$$

Then, we can use $\operatorname{Sup}\left(\widetilde{\alpha}_{i}, \widetilde{\alpha}_{j}\right)$ to denote the support degree between $\widetilde{a}_{i}$ and $\widetilde{\alpha}_{j}$ and $\operatorname{Sup}\left(\widetilde{\alpha}_{i}, \widetilde{\mu}\right)$ to denote the support degree between $\widetilde{\alpha}_{i}$ and $\tilde{\mu}$.

Obviously, $\operatorname{Sup}\left(\widetilde{\alpha}_{i}, \widetilde{\alpha}_{j}\right)$ and $\operatorname{Sup}\left(\widetilde{\alpha}_{i}, \widetilde{\mu}\right)$ satisfy the following properties:

(1) $\operatorname{Sup}\left(\widetilde{\alpha}_{i}, \widetilde{\alpha}_{j}\right) \in[0,1], \operatorname{Sup}\left(\widetilde{\alpha}_{i}, \tilde{\mu}\right) \in[0,1]$;

(2) $\operatorname{Sup}\left(\tilde{\alpha}_{i}, \widetilde{\alpha}_{j}\right)=\operatorname{Sup}\left(\widetilde{\alpha}_{j}, \tilde{\alpha}_{i}\right)$;

(3) $\operatorname{Sup}\left(\widetilde{\alpha}_{i}, \widetilde{\alpha}_{j}\right) \geq \operatorname{Sup}\left(\widetilde{\alpha}_{s}, \widetilde{\alpha}_{p}\right)$ if $d\left(\widetilde{\alpha}_{i}, \widetilde{\alpha}_{j}\right)<d\left(\widetilde{\alpha}_{s}, \widetilde{\alpha}_{p}\right)$, and $\operatorname{Sup}\left(\widetilde{\alpha}_{i}, \widetilde{\mu}\right) \geq \operatorname{Sup}\left(\widetilde{\alpha}_{j}, \widetilde{\mu}\right)$ if $d\left(\widetilde{\alpha}_{i}, \widetilde{\mu}\right)<d\left(\widetilde{\alpha}_{j}, \widetilde{\mu}\right)$, where $d$ is a certain distance measure for interval-valued intuitionistic fuzzy numbers.

Then utilizing hybrid support function in Definition 22, we can manage to obtain the associated argument weights, called power weighting vector, according to

$$
\omega_{j}=\frac{\operatorname{Sup}\left(\widetilde{\alpha}_{j}\right)}{\sum_{j=1}^{n} \operatorname{Sup}\left(\widetilde{\alpha}_{j}\right)}, \quad j=1,2, \ldots, n ;
$$

that is to say, the closer a preference argument is to other arguments or the closer a preference argument is to mid value, the more the argument weighs.

And let $\left(\widetilde{\beta}_{1}, \widetilde{\beta}_{2}, \ldots, \widetilde{\beta}_{n}\right)$ be a permutation of $\left(\widetilde{\alpha}_{1}, \widetilde{\alpha}_{2}, \ldots\right.$, $\left.\widetilde{\alpha}_{n}\right)$, with $\widetilde{\beta}_{j-1} \geq \widetilde{\beta}_{j}$ for all $j=2, \ldots, n$; then we can have the power weighting vector derived according to

$$
\omega_{\beta(j)}=\frac{\operatorname{Sup}\left(\widetilde{\beta}_{j}\right)}{\sum_{j=1}^{n} \operatorname{Sup}\left(\widetilde{\beta}_{j}\right)}, \quad j=1,2, \ldots, n .
$$

Further, we can define the P-GIIFOWA operator and PGIIFOWG operator as follows.
Definition 23. A P-GIIFOWA operator of dimension $n$ is a mapping P-GIIFOWA: $\Omega^{n} \rightarrow \Omega, \omega=\left(\omega_{1}, \omega_{2}, \ldots, \omega_{n}\right)^{T}$ is associated power weighting vector, $\omega_{i} \in[0,1]$ and $\sum_{i=1}^{n} \omega_{i}=$ 1 ; then

$$
\begin{aligned}
& \operatorname{P-GIIFOWA}\left(\widetilde{\alpha}_{1}, \widetilde{\alpha}_{2}, \ldots, \widetilde{\alpha}_{n}\right) \\
& =\left(\frac{\operatorname{Sup}\left(\widetilde{\beta}_{1}\right)}{\sum_{j=1}^{n} \operatorname{Sup}\left(\widetilde{\beta}_{j}\right)} \widetilde{\beta}_{1}^{\lambda} \oplus \frac{\operatorname{Sup}\left(\widetilde{\beta}_{2}\right)}{\sum_{j=1}^{n} \operatorname{Sup}\left(\widetilde{\beta}_{j}\right)} \widetilde{\beta}_{2}^{\lambda}\right. \\
& \left.\quad \oplus \cdots \oplus \frac{\operatorname{Sup}\left(\widetilde{\beta}_{n}\right)}{\sum_{j=1}^{n} \operatorname{Sup}\left(\widetilde{\beta}_{j}\right)} \widetilde{\beta}_{n}^{\lambda}\right)^{1 / \lambda} \\
& =\left(\frac{\operatorname{Sup}\left(\widetilde{\beta}_{1}\right) \widetilde{\beta}_{1}^{\lambda} \oplus \operatorname{Sup}\left(\widetilde{\beta}_{2}\right) \widetilde{\beta}_{2}^{\lambda} \oplus \cdots \oplus \operatorname{Sup}\left(\widetilde{\beta}_{n}\right) \widetilde{\beta}_{n}^{\lambda}}{\sum_{j=1}^{n} \operatorname{Sup}^{\lambda}\left(\widetilde{\beta}_{j}\right)}\right)^{1 / \lambda},
\end{aligned}
$$

where $\left(\widetilde{\beta}_{1}, \widetilde{\beta}_{2}, \ldots, \widetilde{\beta}_{n}\right)$ is a permutation of $\left(\widetilde{\alpha}_{1}, \widetilde{\alpha}_{2}, \ldots, \widetilde{\alpha}_{n}\right)$, with $\widetilde{\beta}_{j-1} \geq \widetilde{\beta}_{j}$ for all $j=2, \ldots, n$.

Definition 24. A P-GIIFOWG operator of dimension $n$ is a mapping P-GIIFOWG: $\Omega^{n} \rightarrow \Omega, \omega=\left(\omega_{1}, \omega_{2}, \ldots, \omega_{n}\right)^{T}$ is associated power weighting vector, $\omega_{i} \in[0,1]$ and $\sum_{i=1}^{n} \omega_{i}=$ 1 ; then

$$
\begin{aligned}
& \text { P-GIIFOWG }\left(\widetilde{\alpha}_{1}, \widetilde{\alpha}_{2}, \ldots, \widetilde{\alpha}_{n}\right) \\
& =\frac{1}{\lambda}\left(\left(\lambda \widetilde{\beta}_{1}\right)^{\operatorname{Sup}\left(\widetilde{\beta}_{1}\right) / \sum_{j=1}^{n} \operatorname{Sup}\left(\widetilde{\beta}_{j}\right)} \otimes\left(\lambda \widetilde{\beta}_{2}\right)^{\operatorname{Sup}\left(\widetilde{\beta}_{2}\right) / \sum_{j=1}^{n} \operatorname{Sup}\left(\widetilde{\beta}_{j}\right)}\right. \\
& \left.\otimes \cdots \otimes\left(\lambda \widetilde{\beta}_{n}\right)^{\operatorname{Sup}\left(\widetilde{\beta}_{n}\right) / \sum_{j=1}^{n} \operatorname{Sup}\left(\tilde{\beta}_{j}\right)}\right) \\
& =\frac{1}{\lambda}\left(\left(\lambda \widetilde{\beta}_{1}\right)^{\operatorname{Sup}\left(\widetilde{\beta}_{1}\right)} \otimes\left(\lambda \widetilde{\beta}_{2}\right)^{\operatorname{Sup}\left(\widetilde{\beta}_{2}\right)}\right. \\
& \left.\otimes \cdots \otimes\left(\lambda \widetilde{\beta}_{n}\right)^{\operatorname{Sup}\left(\widetilde{\beta}_{n}\right)}\right)^{1 / \sum_{j=1}^{n} \operatorname{Sup}\left(\tilde{\beta}_{j}\right)}
\end{aligned}
$$

where $\left(\widetilde{\beta}_{1}, \widetilde{\beta}_{2}, \ldots, \widetilde{\beta}_{n}\right)$ is a permutation of $\left(\widetilde{\alpha}_{1}, \widetilde{\alpha}_{2}, \ldots, \widetilde{\alpha}_{n}\right)$, with $\widetilde{\beta}_{j-1} \geq \widetilde{\beta}_{j}$ for all $j=2, \ldots, n$.

Given $\widetilde{\alpha}_{i}=\left(\left[a_{\widetilde{\alpha}(i)}, b_{\widetilde{\alpha}(i)}\right],\left[c_{\widetilde{\alpha}(i)}, d_{\widetilde{\alpha}(i)}\right]\right), \widetilde{\beta}_{i}=\left(\left[a_{\widetilde{\beta}(i)}, b_{\widetilde{\beta}(i)}\right]\right.$, $\left.\left[c_{\widetilde{\beta}(i)}, d_{\widetilde{\beta}(i)}\right]\right)$, then P-GIIFOWA operator and P-GIIFOWG operator can be transformed into the following forms:

$$
\begin{aligned}
\operatorname{P-GIIFOWA}\left(\widetilde{\alpha}_{1}, \widetilde{\alpha}_{2}, \ldots, \widetilde{\alpha}_{n}\right)= & \left(\left[\left(1-\prod_{j=1}^{n}\left(1-a_{\tilde{\beta}(j)}^{\lambda}\right)^{\omega_{\tilde{\mathcal{\beta}}(j)}}\right)^{1 / \lambda},\right.\right. \\
& \left.\left(1-\prod_{j=1}^{n}\left(1-b_{\tilde{\beta}(j)}^{\lambda}\right)^{\omega_{\tilde{\beta}(j)}}\right)^{1 / \lambda}\right],
\end{aligned}
$$




$$
\begin{aligned}
& {\left[1-\left(1-\prod_{j=1}^{n}\left(1-\left(1-c_{\widetilde{\beta}(j)}\right)^{\lambda}\right)^{\omega_{\widetilde{\beta}(j)}}\right)^{1 / \lambda},\right.} \\
& \left.\left.1-\left(1-\prod_{j=1}^{n}\left(1-\left(1-d_{\tilde{\beta}(j)}\right)^{\lambda}\right)^{\omega_{\widetilde{\beta}(j)}}\right)^{1 / \lambda}\right]\right) \\
& =\left(\left[\left(1-\prod_{j=1}^{n}\left(1-a_{\tilde{\beta}(j)}^{\lambda}\right)^{\operatorname{Sup}\left(\widetilde{\beta}_{j}\right) / \sum_{j=1}^{n} \operatorname{Sup}\left(\widetilde{\beta}_{j}\right)}\right)^{1 / \lambda},\right.\right. \\
& \left.\left(1-\prod_{j=1}^{n}\left(1-b_{\tilde{\beta}(j)}^{\lambda}\right)^{\operatorname{Sup}\left(\tilde{\beta}_{j}\right) / \sum_{j=1}^{n} \operatorname{Sup}\left(\tilde{\beta}_{j}\right)}\right)^{1 / \lambda}\right] \\
& {\left[1-\left(1-\prod_{j=1}^{n}\left(1-\left(1-c_{\widetilde{\beta}(j)}\right)^{\lambda}\right)^{\operatorname{Sup}\left(\widetilde{\beta}_{j}\right) / \sum_{j=1}^{n} \operatorname{Sup}\left(\widetilde{\beta}_{j}\right)}\right)^{1 / \lambda},\right.} \\
& \left.\left.1-\left(1-\prod_{j=1}^{n}\left(1-\left(1-d_{\tilde{\beta}(j)}\right)^{\lambda}\right)^{\operatorname{Sup}\left(\widetilde{\beta}_{j}\right) / \sum_{j=1}^{n} \operatorname{Sup}\left(\widetilde{\beta}_{j}\right)}\right)^{1 / \lambda}\right]\right), \\
& \operatorname{P-GIIFOWG}\left(\widetilde{\alpha}_{1}, \widetilde{\alpha}_{2}, \ldots, \widetilde{\alpha}_{n}\right)=\left(\left[1-\left(1-\prod_{j=1}^{n}\left(1-\left(1-a_{\widetilde{\beta}(j)}\right)^{\lambda}\right)^{\omega_{\widetilde{\beta}(j)}}\right)^{1 / \lambda},\right.\right. \\
& \left.1-\left(1-\prod_{j=1}^{n}\left(1-\left(1-b_{\widetilde{\beta}(j)}\right)^{\lambda}\right)^{\omega_{\tilde{\beta}(j)}}\right)^{1 / \lambda}\right], \\
& {\left[\left(1-\prod_{j=1}^{n}\left(1-c_{\tilde{\beta}(j)}^{\lambda}\right)^{\omega_{\tilde{\beta}(j)}}\right)^{1 / \lambda}\right.} \\
& \left.\left.\left(1-\prod_{j=1}^{n}\left(1-d_{\tilde{\beta}(j)}^{\lambda}\right)^{\omega_{\tilde{\beta}(j)}}\right)^{1 / \lambda}\right]\right) \\
& =\left(\left[1-\left(1-\prod_{j=1}^{n}\left(1-\left(1-a_{\widetilde{\beta}(j)}\right)^{\lambda}\right)^{\operatorname{Sup}\left(\widetilde{\beta}_{j}\right) / \sum_{j=1}^{n} \operatorname{Sup}\left(\tilde{\beta}_{j}\right)}\right)^{1 / \lambda}\right.\right. \text {, } \\
& \left.1-\left(1-\prod_{j=1}^{n}\left(1-\left(1-b_{\widetilde{\beta}(j)}\right)^{\lambda}\right)^{\operatorname{Sup}\left(\widetilde{\beta}_{j}\right) / \sum_{j=1}^{n} \operatorname{Sup}\left(\widetilde{\beta}_{j}\right)}\right)^{1 / \lambda}\right], \\
& {\left[\left(1-\prod_{j=1}^{n}\left(1-c_{\tilde{\beta}(j)}^{\lambda}\right)^{\operatorname{Sup}\left(\tilde{\beta}_{j}\right) / \sum_{j=1}^{n} \operatorname{Sup}\left(\widetilde{\beta}_{j}\right)}\right)^{1 / \lambda}\right.} \\
& \left.\left.\left(1-\prod_{j=1}^{n}\left(1-d_{\tilde{\beta}(j)}^{\lambda}\right)^{\operatorname{Sup}\left(\tilde{\beta}_{j}\right) / \sum_{j=1}^{n} \operatorname{Sup}\left(\tilde{\beta}_{j}\right)}\right)^{1 / \lambda}\right]\right) .
\end{aligned}
$$


By (45), we can have

$$
\begin{aligned}
& \operatorname{P-GIIFOWA}\left(\widetilde{\alpha}_{1}, \widetilde{\alpha}_{2}, \ldots, \widetilde{\alpha}_{n}\right)=\left(\omega_{\widetilde{\beta}(1)} \widetilde{\beta}_{1}^{\lambda} \oplus \omega_{\widetilde{\beta}(2)} \widetilde{\beta}_{2}^{\lambda} \oplus \cdots \oplus \omega_{\widetilde{\beta}(n)} \widetilde{\beta}_{n}^{\lambda}\right)^{1 / \lambda} \\
& =\left(\frac{\sum_{j=1}^{n} \operatorname{Sup}\left(\widetilde{\beta}_{j}\right) \widetilde{\beta}_{j}^{\lambda}}{\sum_{j=1}^{n} \operatorname{Sup}\left(\widetilde{\beta}_{j}\right)}\right)^{1 / \lambda} \\
& =\left(\frac{\sum_{j=1}^{n}\left(\sum_{k=1, j \neq k}^{n}\left(\left(1-d\left(\widetilde{\beta}_{j}, \widetilde{\beta}_{k}\right)\right)+\left(1-d\left(\widetilde{\beta}_{j}, \mu\right)\right)\right)\right) \widetilde{\beta}_{j}^{\lambda}}{\sum_{j=1}^{n} \sum_{k=1, j \neq k}^{n}\left(1-d\left(\widetilde{\beta}_{j}, \widetilde{\beta}_{k}\right)\right)+\left(1-d\left(\widetilde{\beta}_{j}, \mu\right)\right)}\right)^{1 / \lambda}, \\
& \operatorname{P-GIIFOWG}\left(\widetilde{\alpha}_{1}, \widetilde{\alpha}_{2}, \ldots, \widetilde{\alpha}_{n}\right)=\frac{1}{\lambda}\left(\left(\lambda \widetilde{\beta}_{1}\right)^{\omega_{\tilde{\beta}(1)}} \otimes\left(\lambda \widetilde{\beta}_{2}\right)^{\omega_{\tilde{\beta}(2)}} \otimes \cdots \otimes\left(\lambda \widetilde{\beta}_{n}\right)^{\omega_{\tilde{\beta}(n)}}\right) \\
& =\frac{1}{\lambda}\left(\left(\lambda \widetilde{\beta}_{1}\right)^{\operatorname{Sup}\left(\tilde{\beta}_{1}\right)} \otimes\left(\lambda \widetilde{\beta}_{2}\right)^{\operatorname{Sup}\left(\tilde{\beta}_{2}\right)}\right. \\
& \left.\otimes \cdots \otimes\left(\lambda \widetilde{\beta}_{n}\right)^{\operatorname{Sup}\left(\widetilde{\beta}_{n}\right)}\right)^{1 / \sum_{j=1}^{n} \operatorname{Sup}\left(\tilde{\beta}_{j}\right)} \\
& =\frac{1}{\lambda}\left(\prod_{j=1}^{n}\left(\lambda \widetilde{\beta}_{j}\right)^{\sum_{k=1, j \neq k}^{n}\left(1-d\left(\widetilde{\beta}_{j}, \widetilde{\beta}_{k}\right)\right)+\left(1-d\left(\widetilde{\beta}_{j}, \mu\right)\right) / \sum_{j=1}^{n} \sum_{k=1, j \neq k}^{n}\left(1-d\left(\widetilde{\beta}_{j}, \widetilde{\beta}_{k}\right)\right)+\left(1-d\left(\widetilde{\beta}_{j}, \mu\right)\right)}\right) \\
& =\frac{1}{\lambda}\left(\prod_{j=1}^{n}\left(\lambda \widetilde{\beta}_{j}\right)^{\sum_{k=1, j \neq k}^{n}\left(1-d\left(\widetilde{\beta}_{j}, \widetilde{\beta}_{k}\right)\right)+\left(1-d\left(\widetilde{\beta}_{j}, \mu\right)\right)}\right)^{1 / \sum_{j=1}^{n} \sum_{k=1, j \neq k}^{n}\left(1-d\left(\widetilde{\beta}_{j}, \widetilde{\beta}_{k}\right)\right)+\left(1-d\left(\tilde{\beta}_{j}, \mu\right)\right)} .
\end{aligned}
$$

Since

$$
\begin{aligned}
& \sum_{j=1}^{n} \sum_{k=1, j \neq k}^{n}\left(\left(1-d\left(\widetilde{\beta}_{j}, \widetilde{\beta}_{k}\right)\right)+\left(1-d\left(\widetilde{\beta}_{j}, \mu\right)\right)\right) \widetilde{\beta}_{j} \\
& \quad=\sum_{j=1}^{n} \sum_{k=1, j \neq k}^{n}\left(\left(1-d\left(\widetilde{\alpha}_{j}, \widetilde{\alpha}_{k}\right)\right)+\left(1-d\left(\widetilde{\alpha}_{j}, \mu\right)\right)\right) \widetilde{\alpha}_{j}, \\
& \prod_{j=1}^{n}\left(\lambda \widetilde{\beta}_{j}\right)^{\sum_{k=1, j \neq k}^{n}\left(1-d\left(\widetilde{\beta}_{j}, \widetilde{\beta}_{k}\right)\right)+\left(1-d\left(\widetilde{\beta}_{j}, \mu\right)\right)} \\
& =\prod_{j=1}^{n}\left(\lambda \widetilde{\alpha}_{j}\right)^{\sum_{k=1, j \neq k}^{n}\left(1-d\left(\widetilde{\alpha}_{j}, \widetilde{\alpha}_{k}\right)\right)+\left(1-d\left(\widetilde{\alpha}_{j}, \mu\right)\right)}, \\
& \sum_{j=1}^{n} \sum_{k=1, j \neq k}^{n}\left(\left(1-d\left(\widetilde{\beta}_{j}, \widetilde{\beta}_{k}\right)\right)+\left(1-d\left(\widetilde{\beta}_{j}, \mu\right)\right)\right) \\
& =\sum_{j=1}^{n} \sum_{k=1, j \neq k}^{n}\left(\left(1-d\left(\widetilde{\alpha}_{j}, \widetilde{\alpha}_{k}\right)\right)+\left(1-d\left(\widetilde{\alpha}_{j}, \mu\right)\right)\right),
\end{aligned}
$$

then we can have

$$
\begin{aligned}
& \operatorname{P-GIIFOWA}\left(\widetilde{\alpha}_{1}, \widetilde{\alpha}_{2}, \ldots, \widetilde{\alpha}_{n}\right) \\
& =\left(\omega_{\widetilde{\alpha}(1)} \widetilde{\alpha}_{1}^{\lambda} \oplus \omega_{\widetilde{\alpha}(2)} \widetilde{\alpha}_{2}^{\lambda} \oplus \cdots \oplus \omega_{\widetilde{\alpha}(n)} \widetilde{\alpha}_{n}^{\lambda}\right)^{1 / \lambda}, \\
& \text { P-GIIFOWG }\left(\widetilde{\alpha}_{1}, \widetilde{\alpha}_{2}, \ldots, \widetilde{\alpha}_{n}\right) \\
& =\frac{1}{\lambda}\left(\left(\lambda \widetilde{\alpha}_{1}\right)^{\left.\omega_{\widetilde{\alpha}(1)} \otimes\left(\lambda \widetilde{\alpha}_{2}\right)^{\omega_{\tilde{\alpha}(2)}} \otimes \cdots \otimes\left(\lambda \widetilde{\alpha}_{n}\right)^{\omega_{\widetilde{\alpha}(n)}}\right) .}\right.
\end{aligned}
$$

Obviously, P-GIIFOWA and P-GIIFOWG are also neat and dependent operators.

Theorem 25. Let $\left(\widetilde{\alpha}_{1}, \widetilde{\alpha}_{2}, \ldots, \widetilde{\alpha}_{n}\right)$ be a collection of intervalvalued intuitionistic fuzzy arguments, and $\left(\widetilde{\beta}_{1}, \widetilde{\beta}_{2}, \ldots, \widetilde{\beta}_{n}\right)$ is a permutation of $\left(\widetilde{\alpha}_{1}, \widetilde{\alpha}_{2}, \ldots, \widetilde{\alpha}_{n}\right)$, with $\widetilde{\beta}_{j-1} \geq \widetilde{\beta}_{j}$ for all $j=$ $2, \ldots, n$. If $\operatorname{Sup}\left(\widetilde{\beta}_{i}\right) \geq \operatorname{Sup}\left(\widetilde{\beta}_{j}\right)$; then $\omega_{\widetilde{\beta}(i)} \geq \omega_{\widetilde{\beta}(j)}$.

Theorem 26. Let $\widetilde{\alpha}_{j}=\left(\left[a_{j}, b_{j}\right],\left[c_{j}, d_{j}\right]\right)(j=1,2, \ldots, n)$ be a collection of interval-valued intuitionistic fuzzy arguments, and $\omega=\left(\omega_{1}, \omega_{2}, \ldots, \omega_{n}\right)^{T}$ the weighting vector derived by hybrid support method related to the P-GIIFOWA operator and $P$-GIIFOWG operator, with $\omega_{j} \in[0,1]$ and $\sum_{j=1}^{n} \omega_{j}=1$; then 
the P-GIIFOWA operator and the P-GIIFOWG operator have the following properties.

(1) Commutativity: let $\left(\widetilde{\alpha}_{1}^{*}, \widetilde{\alpha}_{2}^{*}, \ldots, \widetilde{\alpha}_{n}^{*}\right)$ be any a permutation of $\left(\widetilde{\alpha}_{1}, \widetilde{\alpha}_{2}, \ldots, \widetilde{\alpha}_{n}\right)$; then

$$
\begin{aligned}
& P \text {-GIIFOWA } A_{\omega, \lambda}\left(\widetilde{\alpha}_{1}^{*}, \widetilde{\alpha}_{2}^{*}, \ldots, \widetilde{\alpha}_{n}^{*}\right) \\
& \quad=P-G I I F O W A_{\omega, \lambda}\left(\widetilde{\alpha}_{1}, \widetilde{\alpha}_{2}, \ldots \widetilde{\alpha}_{n}\right), \\
& P-G I I F O W G_{\omega, \lambda}\left(\widetilde{\alpha}_{1}^{*}, \widetilde{\alpha}_{2}^{*}, \ldots, \widetilde{\alpha}_{n}^{*}\right) \\
& \quad=P-G I I F O W G_{\omega, \lambda}\left(\widetilde{\alpha}_{1}, \widetilde{\alpha}_{2}, \ldots, \widetilde{\alpha}_{n}\right) .
\end{aligned}
$$

(2) Idempotency: let $\tilde{\alpha}_{j}=\tilde{\alpha}$, for all $j=1,2, \ldots, n$; then

$$
\begin{aligned}
& P-G I I F O W A_{\omega, \lambda}\left(\widetilde{\alpha}_{1}, \widetilde{\alpha}_{2}, \ldots, \widetilde{\alpha}_{n}\right)=\widetilde{\alpha}, \\
& P-G I I F O W G_{\omega, \lambda}\left(\widetilde{\alpha}_{1}, \widetilde{\alpha}_{2}, \ldots, \widetilde{\alpha}_{n}\right)=\widetilde{\alpha} .
\end{aligned}
$$

(3) Boundedness: the P-GIIFOWA operator and the PGIIFOWG operator lie between the $\max$ and $\min$ operators:

$$
\begin{aligned}
& \tilde{\alpha}^{-} \leq \text {P-GIIFOWA }{ }_{\omega, \lambda}\left(\widetilde{\alpha}_{1}, \widetilde{\alpha}_{2}, \ldots, \widetilde{\alpha}_{n}\right) \leq \tilde{\alpha}^{+}, \\
& \tilde{\alpha}^{-} \leq P-G I I F O W G_{\omega, \lambda}\left(\widetilde{\alpha}_{1}, \widetilde{\alpha}_{2}, \ldots, \widetilde{\alpha}_{n}\right) \leq \tilde{\alpha}^{+},
\end{aligned}
$$

where

$$
\begin{aligned}
& \widetilde{\alpha}^{-}=\left(\left[\min _{j}\left(a_{j}\right), \min _{j}\left(b_{j}\right)\right],\left[\max _{j}\left(c_{j}\right), \max _{j}\left(d_{j}\right)\right]\right), \\
& \tilde{\alpha}^{+}=\left(\left[\max _{j}\left(a_{j}\right), \max _{j}\left(b_{j}\right)\right],\left[\min _{j}\left(c_{j}\right), \min _{j}\left(d_{j}\right)\right]\right) .
\end{aligned}
$$

Theorem 27. Let $\tilde{\alpha}_{j}=\left(\left[a_{\widetilde{\alpha}(j)}, b_{\widetilde{\alpha}(j)}\right],\left[c_{\widetilde{\alpha}(j)}, d_{\widetilde{\alpha}(j)}\right]\right), \tilde{\beta}_{j}=$ $\left(\left[a_{\widetilde{\beta}(j)}, b_{\widetilde{\beta}(j)}\right],\left[c_{\widetilde{\beta}(j)}, d_{\widetilde{\beta}(j)}\right]\right)$ be two collections of interval-valued intuitionistic fuzzy arguments, $\left(\widetilde{\beta}_{1}, \widetilde{\beta}_{2}, \ldots, \widetilde{\beta}_{n}\right)$ a permutation of $\left(\widetilde{\alpha}_{1}, \widetilde{\alpha}_{2}, \ldots, \widetilde{\alpha}_{n}\right)$, with $\widetilde{\beta}_{j-1} \geq \widetilde{\beta}_{j}$ for all $j=2, \ldots, n$, and $\omega=\left(\omega_{1}, \omega_{2}, \ldots, \omega_{n}\right)^{T}$ the weighting vector derived by hybrid support method related to the P-GIIFOWA operator and PGIIFOWG operator, $\omega_{j} \in[0,1], \sum_{j=1}^{n} \omega_{j}=1$; then

(1) if $\lambda=1$, then the P-GIIFOWA operator and $P$ GIIFOWG operator reduce to the following P-IIFOWA operator and P-IIFOWG operator:

$$
\begin{aligned}
& \operatorname{P-IIFOWA}\left(\widetilde{\alpha}_{1}, \widetilde{\alpha}_{2}, \ldots, \widetilde{\alpha}_{n}\right) \\
& =\frac{\operatorname{Sup}\left(\widetilde{\beta}_{1}\right)}{\sum_{j=1}^{n} \operatorname{Sup}\left(\widetilde{\beta}_{j}\right)} \widetilde{\beta}_{1} \oplus \frac{\operatorname{Sup}\left(\widetilde{\beta}_{2}\right)}{\sum_{j=1}^{n} \operatorname{Sup}\left(\widetilde{\beta}_{j}\right)} \widetilde{\beta}_{2}
\end{aligned}
$$

$$
\begin{aligned}
& \oplus \cdots \oplus \frac{\operatorname{Sup}\left(\widetilde{\beta}_{n}\right)}{\sum_{j=1}^{n} \operatorname{Sup}\left(\widetilde{\beta}_{j}\right)} \widetilde{\beta}_{n} \\
& =\left(\left[1-\prod_{j=1}^{n}\left(1-a_{\tilde{\beta}(j)}\right)^{\operatorname{Sup}\left(\widetilde{\beta}_{j}\right) / \sum_{j=1}^{n} \operatorname{Sup}\left(\widetilde{\beta}_{j}\right)}\right.\right. \text {, } \\
& \left.1-\prod_{j=1}^{n}\left(1-b_{\widetilde{\beta}(j)}\right)^{\operatorname{Sup}\left(\widetilde{\beta}_{j}\right) / \sum_{j=1}^{n} \operatorname{Sup}\left(\widetilde{\beta}_{j}\right)}\right],
\end{aligned}
$$

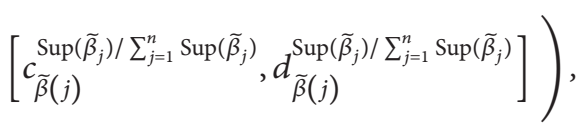

$$
\begin{aligned}
& P- \operatorname{IIFOWG}\left(\widetilde{\alpha}_{1}, \widetilde{\alpha}_{2}, \ldots, \widetilde{\alpha}_{n}\right) \\
&=\left(\widetilde{\beta}_{1}^{\operatorname{Sup}\left(\widetilde{\beta}_{1}\right)} \otimes \widetilde{\beta}_{2}^{\operatorname{Sup}\left(\widetilde{\beta}_{2}\right)} \otimes \cdots \otimes \widetilde{\beta}_{n}^{\operatorname{Sup}\left(\widetilde{\beta}_{n}\right)}\right)^{1 / \sum_{j=1}^{n} \operatorname{Sup}\left(\widetilde{\beta}_{j}\right)} \\
&= {\left[a_{\widetilde{\beta}(j)}^{\operatorname{Sup}\left(\widetilde{\beta}_{j}\right) / \sum_{j=1}^{n} \operatorname{Sup}\left(\widetilde{\beta}_{j}\right)}, b_{\widetilde{\beta}(j)}^{\operatorname{Sup}\left(\widetilde{\beta}_{j}\right) / \sum_{j=1}^{n} \operatorname{Sup}\left(\widetilde{\beta}_{j}\right)}\right], } \\
& {\left[1-\prod_{j=1}^{n}\left(1-c_{\widetilde{\beta}(j)}\right)^{\operatorname{Sup}\left(\widetilde{\beta}_{j}\right) / \sum_{j=1}^{n} \operatorname{Sup}\left(\widetilde{\beta}_{j}\right)},\right.} \\
&\left.\left.1-\prod_{j=1}^{n}\left(1-d_{\widetilde{\beta}(j)}^{\lambda}\right)^{\operatorname{Sup}\left(\widetilde{\beta}_{j}\right) / \sum_{j=1}^{n} \operatorname{Sup}\left(\widetilde{\beta}_{j}\right)}\right]\right) ;
\end{aligned}
$$

(2) if $\lambda \rightarrow 0$, then the P-GIIFOWA operator reduces to the $P$-IIFOWG operator;

(3) if $\omega=(1 / n, 1 / n, \ldots, 1 / n)^{T}$, then the P-GIIFOWA operator and P-GIIFOWG operator reduce to the GIIFA operator and GIIFG operator;

(4) if $\omega=(1 / n, 1 / n, \ldots, 1 / n)^{T}$ and $\lambda=1$, then the $P$ GIIFOWA operator and P-GIIFOWG operator reduce to the IIFA operator and IIFG operator;

(5) if $\omega=(1 / n, 1 / n, \ldots, 1 / n)^{T}$ and $\lambda \rightarrow 0$, then the $P$ GIIFOWA operator reduces to the IIFG operator.

Theorem 28. Let $\widetilde{\alpha}_{j}=\left(\left[a_{\widetilde{\alpha}(j)}, b_{\widetilde{\alpha}(j)}\right],\left[c_{\widetilde{\alpha}(j)}, d_{\widetilde{\alpha}(j)}\right]\right), \widetilde{\beta}_{j}=$ $\left(\left[a_{\widetilde{\beta}(j)}, b_{\widetilde{\beta}(j)}\right],\left[c_{\widetilde{\beta}(j)}, d_{\widetilde{\beta}(j)}\right]\right)$ be two collections of interval-valued intuitionistic fuzzy arguments, $\left(\widetilde{\beta}_{1}, \widetilde{\beta}_{2}, \ldots, \widetilde{\beta}_{n}\right)$ a permutation of $\left(\widetilde{\alpha}_{1}, \widetilde{\alpha}_{2}, \ldots, \widetilde{\alpha}_{n}\right)$, with $\widetilde{\beta}_{j-1} \geq \widetilde{\beta}_{j}$ for all $j=2, \ldots, n, \omega=$ $\left(\omega_{1}, \omega_{2}, \ldots, \omega_{n}\right)^{T}$ the weight vector derived by hybrid support method related to the P-GIIFOWA operator and P-GIIFOWG operator, with $\omega_{j} \in[0,1]$ and $\sum_{j=1}^{n} \omega_{j}=1$; then

(1) $P$-IIFOWG $\left(\widetilde{\alpha}_{1}, \widetilde{\alpha}_{2}, \ldots, \widetilde{\alpha}_{n}\right) \leq P-\operatorname{IIFOWA}\left(\widetilde{\alpha}_{1}, \widetilde{\alpha}_{2}, \ldots\right.$, $\left.\widetilde{\alpha}_{n}\right)$

(2) P-IIFOWG $\left(\widetilde{\alpha}_{1}, \widetilde{\alpha}_{2}, \ldots, \widetilde{\alpha}_{n}\right) \leq P-G I I F O W A_{\lambda}\left(\widetilde{\alpha}_{1}, \widetilde{\alpha}_{2}\right.$, $\left.\ldots, \widetilde{\alpha}_{n}\right)$;

(3) $P$-GIIFOWG ${ }_{\lambda}\left(\widetilde{\alpha}_{1}, \widetilde{\alpha}_{2}, \ldots, \widetilde{\alpha}_{n}\right) \leq \operatorname{P-IFOWA}\left(\widetilde{\alpha}_{1}, \widetilde{\alpha}_{2}\right.$, $\left.\ldots, \widetilde{\alpha}_{n}\right)$. 
Proof. Similar to the proof of Theorem 20, Theorem 28 can be proved by mathematical induction method, so proof steps are omitted here.

Example 29. For a group decision making problem, suppose that there are six decision makers $d_{j}(j=1,2, \ldots, 6)$ to provide their individual preferences with interval-valued intuitionistic fuzzy numbers. Then, the preference arguments can be collected as follows:

$$
\begin{aligned}
& \widetilde{\alpha}_{1}=([0.5,0.6],[0.2,0.4]), \\
& \widetilde{\alpha}_{2}=([0.3,0.6],[0.3,0.4]), \\
& \widetilde{\alpha}_{3}=([0.4,0.7],[0.2,0.3]), \\
& \widetilde{\alpha}_{4}=([0.3,0.5],[0.1,0.4]), \\
& \widetilde{\alpha}_{5}=([0.4,0.7],[0.2,0.3]), \\
& \widetilde{\alpha}_{6}=([0.6,0.8],[0.0,0.2]) .
\end{aligned}
$$

According to (44) and (45), we can have the power weighting vector:

$$
\omega=\left(\omega_{1}, \omega_{2}, \omega_{3}, \omega_{4}, \omega_{5}, \omega_{6}\right),
$$

where $\omega_{1}=0.1653, \omega_{2}=0.164, \omega_{3}=0.1715, \omega_{4}=0.1651$, $\omega_{5}=0.1715$, and $\omega_{6}=0.1625$.

Suppose $\lambda=5$, then according to (51) and (52), it follows that

$$
\begin{aligned}
& \text { P-GIIFOWA }\left(\widetilde{\alpha}_{1}, \widetilde{\alpha}_{2}, \ldots, \widetilde{\alpha}_{n}\right) \\
& \quad=([0.4691,0.6828],[0.0,0.299]), \\
& \text { P-GIIFOWG }\left(\widetilde{\alpha}_{1}, \widetilde{\alpha}_{2}, \ldots, \widetilde{\alpha}_{n}\right) \\
& \quad=([0.3808,0.6049],[0.2225,0.3422]) .
\end{aligned}
$$

Theorem 30. Let $\widetilde{\alpha}_{j}=\left(\left[a_{\widetilde{\alpha}(j)}, b_{\widetilde{\alpha}(j)}\right],\left[c_{\widetilde{\alpha}(j)}, d_{\widetilde{\alpha}(j)}\right]\right)$ and $\widetilde{\beta}_{j}=$ $\left(\left[a_{\tilde{\beta}(j)}, b_{\widetilde{\beta}(j)}\right],\left[c_{\widetilde{\beta}(j)}, d_{\widetilde{\beta}(j)}\right]\right)$ be two collections of interval-valued intuitionistic fuzzy arguments, and let $\widetilde{\gamma}$ be the interval-valued intuitionistic fuzzy number obtained by applying GIIFOWA $\lambda_{\lambda}$ or GIIFOWG $G_{\lambda}$ on $\widetilde{\alpha}_{j}$ and $\widetilde{\beta}_{j}$; then one can have

(1-a) if $c_{\widetilde{\beta}(j)}=0, \tilde{\gamma}=\operatorname{GIIFOWA}\left(\widetilde{\alpha}_{j}, \widetilde{\beta}_{j}\right)=([\bar{a}, \bar{b}],[\bar{c}, \bar{d}])$, then $\bar{c}=0$;

(1-b) if $d_{\widetilde{\beta}(j)}=0, \widetilde{\gamma}=\operatorname{GIFOWA}_{\lambda}\left(\widetilde{\alpha}_{j}, \widetilde{\beta}_{j}\right)=([\bar{a}, \bar{b}],[\bar{c}, \bar{d}])$, then $\bar{d}=0$;

(1-c) if $c_{\widetilde{\beta}(j)}=0$ and $d_{\widetilde{\beta}(j)}=0, \widetilde{\gamma}=\operatorname{GIIFOWA} A_{\lambda}\left(\widetilde{\alpha}_{j}, \widetilde{\beta}_{j}\right)=$ $([\bar{a}, \bar{b}],[\bar{c}, \bar{d}])$, then $\bar{c}=\bar{d}=0$;

(2-a) if $a_{\widetilde{\beta}(j)}=0, \widetilde{\gamma}=\operatorname{GIIFOWA}_{\lambda}\left(\widetilde{\alpha}_{j}, \widetilde{\beta}_{j}\right)=([\bar{a}, \bar{b}],[\bar{c}, \bar{d}])$, then $\bar{a}=0$;

(2-b) if $b_{\widetilde{\beta}(j)}=0, \tilde{\gamma}=\operatorname{GIIFOWA}_{\lambda}\left(\widetilde{\alpha}_{j}, \widetilde{\beta}_{j}\right)=([\bar{a}, \bar{b}],[\bar{c}, \bar{d}])$, then $\bar{b}=0$;

$(2-c)$ if $a_{\widetilde{\beta}(j)}=0$ and $b_{\widetilde{\beta}(j)}=0, \tilde{\gamma}=\operatorname{GIIFOWA} A_{\lambda}\left(\widetilde{\alpha}_{j}, \widetilde{\beta}_{j}\right)=$ $([\bar{a}, \bar{b}],[\bar{c}, \bar{d}])$, then $\bar{a}=\bar{b}=0$.
Proof. For the proposition (1-a), if $c_{\beta(j)}=0$, then we can have

$$
\begin{aligned}
& \operatorname{GIIFOWA}_{\lambda}\left(\widetilde{\alpha}_{1}, \widetilde{\alpha}_{2}, \ldots, \widetilde{\alpha}_{n}\right) \\
& =\left({\underset{j=1}{n}}_{j=1}^{n}\left(w_{j} \widetilde{\beta}_{j}^{\lambda}\right)\right)^{1 / \lambda} \\
& =\left(\left[\left(1-\prod_{j=1}^{n}\left(1-a_{\tilde{\beta}(j)}^{\lambda}\right)^{w_{j}}\right)^{1 / \lambda},\right.\right. \\
& \left.\left(1-\prod_{j=1}^{n}\left(1-b_{\widetilde{\beta}(j)}^{\lambda}\right)^{w_{j}}\right)^{1 / \lambda}\right] \\
& {\left[1-\left(1-\prod_{j=1}^{n}\left(1-\left(1-c_{\widetilde{\beta}(j)}\right)^{\lambda}\right)^{w_{j}}\right)^{1 / \lambda},\right.} \\
& \left.\left.1-\left(1-\prod_{j=1}^{n}\left(1-\left(1-d_{\widetilde{\beta}(j)}\right)^{\lambda}\right)^{w_{j}}\right)^{1 / \lambda}\right]\right) \\
& =([\bar{a}, \bar{b}],[0, \bar{d}]),
\end{aligned}
$$

so the proposition (1-a) is right. Correspondingly, proposition (1-b) and proposition (1-c) can be proved in the same way.

For the proposition (2-a), if $a_{\beta(j)}=0$, then

$$
\begin{aligned}
& \operatorname{GIIFOWG}_{\lambda}\left(\widetilde{\alpha}_{1}, \widetilde{\alpha}_{2}, \ldots, \widetilde{\alpha}_{n}\right) \\
& =\frac{1}{\lambda}\left(\bigotimes_{j=1}^{n}\left(\lambda \widetilde{\beta}_{j}\right)^{w_{j}}\right) \\
& =\left(\left[1-\left(1-\prod_{j=1}^{n}\left(1-\left(1-a_{\widetilde{\beta}(j)}\right)^{\lambda}\right)^{w_{j}}\right)^{1 / \lambda},\right.\right. \\
& \left.1-\left(1-\prod_{j=1}^{n}\left(1-\left(1-b_{\widetilde{\beta}(j)}\right)^{\lambda}\right)^{w_{j}}\right)^{1 / \lambda}\right], \\
& {\left[\left(1-\prod_{j=1}^{n}\left(1-c_{\widetilde{\beta}(j)}^{\lambda}\right)^{w_{j}}\right)^{1 / \lambda},\right.} \\
& \left.\left.\left(1-\prod_{j=1}^{n}\left(1-d_{\tilde{\beta}(j)}^{\lambda}\right)^{w_{j}}\right)^{1 / \lambda}\right]\right) \\
& =([0, \bar{b}],[\bar{c}, \bar{d}]) \text {, }
\end{aligned}
$$

so the proposition (2-a) is right, and proposition (2-b) and proposition $(2-c)$ can also be proved similarly.

Thus, according to Theorem 30, for the situation that $c_{\widetilde{\beta}(j)}=0$ or $d_{\widetilde{\beta}(j)}=0$, GIIFOWG ${ }_{\lambda}$ operators should be 
better choices than GIIFOWA $\lambda$ operators to consider more completely the preference information indicated by nonzero arguments, while for the situation $a_{\tilde{\beta}(j)}=0$ or $b_{\widetilde{\beta}(j)}=0$, GIIFOWA $_{\lambda}$ operators can use preference information more completely than GIIFOWG $\boldsymbol{\lambda}_{\lambda}$ operators.

\section{An Approach for Multiple Attribute Group Decision Making with Interval-Valued Intuitionistic Fuzzy Information}

For the multiple attribute group decision making problems, in which both the attribute weights and the expert weights take the form of real numbers, and the attribute arguments take the form of interval-valued intuitionistic fuzzy numbers, we develop a decision making approach based on the above-presented dependent interval-valued intuitionistic fuzzy aggregation operators.

Let $X=\left\{x_{1}, x_{2}, \ldots, x_{n}\right\}$ be a set of alternatives, $G=$ $\left\{g_{1}, g_{2}, \ldots, g_{m}\right\}$ a set of attributes, $\omega=\left\{\omega_{1}, \omega_{2}, \ldots, \omega_{m}\right\}^{T}$ the weighting vector of attributes, where $\omega_{j} \in[0,1], \sum_{j=1}^{n} \omega_{j}=$ $1, D=\left\{d_{1}, d_{2}, \ldots, d_{t}\right\}$ a set of decision makers, and $\lambda=$ $\left(\lambda^{(1)}, \lambda^{(2)}, \ldots, \lambda^{(t)}\right)$ the weighting vector of decision makers. The proposed approach involves the following steps.

Step 1. Construct individual interval-valued intuitionistic fuzzy evaluation matrices $\widetilde{R}^{(k)} \cdot \widetilde{R}^{(k)}=\left(\widetilde{r}_{i j}^{(k)}\right)_{n \times m}=$ $\left(\widetilde{\mu}_{i j}^{(k)}, \widetilde{v}_{i j}^{(k)}\right)_{n \times m}=\left(\left[\mu_{i j}^{L(k)}, \mu_{i j}^{U(k)}\right],\left[v_{i j}^{L(k)}, v_{i j}^{U(k)}\right]\right)_{n \times m}$, where $\left[\mu_{i j}^{L(k)}\right.$, $\left.\mu_{i j}^{U(k)}\right]$ indicates the degree to which the alternative $x_{i}$ satisfies the attribute $g_{j},\left[v_{i j}^{L(k)}, v_{i j}^{U(k)}\right]$ indicates the degree to which the alternative $x_{i}(i=1,2, \ldots, n)$ does not satisfies the attribute $g_{j}(j=1,2, \ldots, m)$.

Step 2. Calculate argument weighting vector $\omega^{(k)}=\left(\omega_{1}^{(k)}\right.$, $\left.\omega_{2}^{(k)}, \ldots, \omega_{n}^{(k)}\right)^{T}$ associated with the interval-valued intuitionistic fuzzy value $\widetilde{r}_{i j}^{(k)}$ in $k$ th individual matrix $\widetilde{R}^{(k)}$ according to $(24)$ or $(46)$.

Step 3. Utilize Gaussian-GIIFOWA operator, P-GIIFOWA operator, Gaussian-GIIFOWG operator, or P-GIIFOWG operator to aggregate the arguments in $i$ th row of $k$ th decision maker's assessment matrix $\widetilde{R}^{(k)}$ as the corresponding intervalvalued intuitionistic fuzzy value $\widetilde{r}_{i k}$ in the group decision matrix $\dot{R}$ for each $x_{i}$.

Step 4. Utilize IIFWA operator or IIFWG operator to derive the overall group interval-valued intuitionistic fuzzy decision vector $\tilde{r}$ for all the alternatives by aggregating the values in each row of $\dot{R}$.

Step 5. Calculate score values $s\left(\widetilde{r}_{i}\right)(i=1,2, \ldots, n)$ and accuracy values $h\left(\widetilde{r}_{i}\right)(i=1,2, \ldots, n)$ of alternative $x_{i}$ and then rank all the alternatives to select the optimal one(s) according to Definition 5.

Step 6. End.

\section{Application to Exploitation Investment Evaluation of Tourist Spots}

5.1. Application Study. Suppose that a tourism development and investment company is about to choose the most desirable project(s) to invest from several candidate tourist spots, which are filtered out through initial screening and advance to an investment expert committee for detailed comprehensive due diligence, such as evaluation of exploitation feasibility and evaluation of sustainable management strategies [69]. Given that five filtered alternative tourist spots $x_{i}(i=1,2,3,4,5)$ advance to be reviewed for acceptance, the corresponding investment criteria about exploitation feasibility of tourist spots could be constructed according to [69] from the following five aspects: variety $\left(g_{1}\right)$, orientability $\left(g_{2}\right)$, monopoly $\left(g_{3}\right)$, destructibility $\left(g_{4}\right)$, and novelty $\left(g_{5}\right)$. And three domain experts are organized as decision makers DM $d_{k}(k=1,2,3)$ in the investment expert committee to assess alternative tourist spots $x_{i}$ by interval-valued intuitionistic fuzzy numbers with respect to each investment criterion $g_{j}$. Suppose the decision makers' weighting vector $\lambda=(0.3,0.3,0.4)^{T}$. According to Section 4, the procedure for solving this practical MAGDM problem contains the following steps.

Step 1. According to the opinions of decision makers, the interval-valued intuitionistic fuzzy decision matrix $\widetilde{R}^{(k)}=$ $\left(\widetilde{r}_{i j}^{(k)}\right)_{n \times m}(k=1,2,3)$ can be firstly constructed, and the assessments are listed in Tables 1, 2, and 3.

Step 2. Respectively calculate Gaussian weighting vector according to (24) and power weighting vector according to (46).

Gaussian weighting vector:

$$
\begin{aligned}
& \omega^{(1)}=(0.2443,0.159,0.2682,0.1661,0.1623)^{T}, \\
& \omega^{(2)}=(0.1719,0.2185,0.3227,0.1169,0.17)^{T}, \\
& \omega^{(3)}=(0.1613,0.2245,0.2058,0.2721,0.1363)^{T} ;
\end{aligned}
$$

power weighting vector:

$$
\begin{gathered}
\omega^{(1)}=(0.2022,0.197,0.2046,0.1976,0.1985)^{T}, \\
\omega^{(2)}=(0.1982,0.2030,0.2072,0.1901,0.2015)^{T}, \\
\omega^{(3)}=(0.1972,0.2041,0.2029,0.2069,0.1889)^{T} .
\end{gathered}
$$

Step 3. Then, respectively utilize the Gaussian-GIIFOWA operator, P-GIIFOWA operator, Gaussian-GIIFOWG operator, or P-GIIFOWG operator to aggregate each intervalvalued intuitionistic fuzzy arguments in $i$ th row of $k$ th decision maker's assessment matrix $\widetilde{R}^{(k)}$ and get the group decision matrix $\dot{R}$ for each $x_{i}$. Here, suppose $\lambda=1$, and the results are shown in Tables 4, 5, 6, and 7.

Step 4. Aggregate each row in $\dot{R}$ using IIFWA operator or IIFWG operator to derive the interval-valued intuitionistic 
TABLE 1: Decision matrix $\widetilde{R}^{(1)}$ by $d_{1}$.

\begin{tabular}{cccccc}
\hline & $g_{1}$ & $g_{2}$ & $g_{3}$ & $g_{4}$ & $g_{5}$ \\
\hline$x_{1}$ & $([0.4,0.5],[0.3,0.4])$ & $([0.5,0.6],[0.1,0.2])$ & $([0.6,0.7],[0.2,0.3])$ & $([0.7,0.8],[0.1,0.2])$ & $([0.7,0.8],[0.0,0.2])$ \\
$x_{2}$ & $([0.6,0.8],[0.1,0.2])$ & $([0.5,0.6],[0.3,0.4])$ & $([0.4,0.5],[0.3,0.4])$ & $([0.4,0.6],[0.3,0.4])$ & $([0.4,0.7],[0.1,0.3])$ \\
$x_{3}$ & $([0.5,0.6],[0.3,0.4])$ & $([0.5,0.7],[0.1,0.2])$ & $([0.5,0.6],[0.3,0.4])$ & $([0.3,0.4],[0.2,0.5])$ & $([0.6,0.7],[0.2,0.3])$ \\
$x_{4}$ & $([0.5,0.6],[0.3,0.4])$ & $([0.7,0.8],[0.0,0.1])$ & $([0.4,0.5],[0.2,0.4])$ & $([0.5,0.7],[0.1,0.2])$ & $([0.5,0.7],[0.2,0.3])$ \\
$x_{5}$ & $([0.4,0.7],[0.2,0.3])$ & $([0.5,0.6],[0.2,0.4])$ & $([0.3,0.6],[0.3,0.4])$ & $([0.6,0.8],[0.1,0.2])$ & $([0.4,0.5],[0.2,0.3])$ \\
\hline
\end{tabular}

TABLE 2: Decision matrix $\widetilde{R}^{(2)}$ by $d_{2}$.

\begin{tabular}{cccccc}
\hline & $g_{1}$ & $g_{2}$ & $g_{3}$ & $g_{4}$ & $g_{5}$ \\
\hline$x_{1}$ & $([0.4,0.6],[0.3,0.4])$ & $([0.5,0.7],[0.0,0.2])$ & $([0.5,0.6],[0.2,0.4])$ & $([0.6,0.8],[0.1,0.2])$ & $([0.4,0.7],[0.2,0.3])$ \\
$x_{2}$ & $([0.5,0.8],[0.1,0.2])$ & $([0.3,0.5],[0.2,0.3])$ & $([0.3,0.6],[0.2,0.4])$ & $([0.4,0.5],[0.2,0.4])$ & $([0.3,0.6],[0.2,0.3])$ \\
$x_{3}$ & $([0.5,0.6],[0.0,0.1])$ & $([0.5,0.8],[0.1,0.2])$ & $([0.4,0.7],[0.2,0.3])$ & $([0.2,0.4],[0.2,0.3])$ & $([0.5,0.8],[0.0,0.2])$ \\
$x_{4}$ & $([0.5,0.7],[0.1,0.3])$ & $([0.4,0.6],[0.0,0.1])$ & $([0.3,0.5],[0.2,0.4])$ & $([0.7,0.9],[0.0,0.1])$ & $([0.3,0.5],[0.2,0.2])$ \\
$x_{5}$ & $([0.7,0.8],[0.0,0.1])$ & $([0.4,0.6],[0.0,0.2])$ & $([0.4,0.7],[0.2,0.3])$ & $([0.3,0.5],[0.1,0.3])$ & $([0.6,0.7],[0.1,0.2])$ \\
\hline
\end{tabular}

TABLE 3: Decision matrix $\widetilde{R}^{(3)}$ by $d_{3}$.

\begin{tabular}{cccccc}
\hline & $g_{1}$ & $g_{2}$ & $g_{3}$ & $g_{4}$ & $g_{5}$ \\
\hline$x_{1}$ & $([0.3,0.4],[0.4,0.5])$ & $([0.8,0.9],[0.1,0.1])$ & $([0.7,0.8],[0.1,0.2])$ & $([0.4,0.5],[0.3,0.5])$ & $([0.2,0.4],[0.3,0.6])$ \\
$x_{2}$ & $([0.5,0.7],[0.1,0.3])$ & $([0.4,0.7],[0.2,0.3])$ & $([0.4,0.5],[0.2,0.2])$ & $([0.6,0.8],[0.1,0.2])$ & $([0.2,0.3],[0.0,0.1])$ \\
$x_{3}$ & $([0.2,0.4],[0.1,0.2])$ & $([0.4,0.5],[0.2,0.4])$ & $([0.5,0.8],[0.0,0.1])$ & $([0.4,0.6],[0.2,0.3])$ & $([0.5,0.6],[0.2,0.3])$ \\
$x_{4}$ & $([0.7,0.8],[0.0,0.2])$ & $([0.5,0.7],[0.1,0.2])$ & $([0.6,0.7],[0.1,0.3])$ & $([0.4,0.5],[0.1,0.2])$ & $([0.7,0.8],[0.1,0.2])$ \\
$x_{5}$ & $([0.5,0.6],[0.2,0.4])$ & $([0.5,0.8],[0.0,0.2])$ & $([0.4,0.7],[0.2,0.3])$ & $([0.3,0.6],[0.2,0.3])$ & $([0.7,0.8],[0.0,0.1])$ \\
\hline
\end{tabular}

TABLE 4: Group decision matrix $\dot{R}$ obtained by utilizing Gaussian-GIIFOWA operator.

\begin{tabular}{cccc}
\hline & $d_{1}$ & $d_{2}$ & $d_{3}$ \\
\hline$x_{1}$ & $([0.5836,0.6885],[0.0,0.2642])$ & $([0.4815,0.6701],[0.0,0.3019])$ & $([0.5666,0.6954],[0.1959,0.2958])$ \\
$x_{2}$ & $([0.4721,0.6578],[0.1919,0.3223])$ & $([0.3511,0.6173],[0.1775,0.3175])$ & $([0.4574,0.6650],[0.0,0.2128])$ \\
$x_{3}$ & $([0.4900,0.6099],[0.2205,0.3549])$ & $([0.4397,0.7080],[0.0,0.2122])$ & $([0.4095,0.6107],[0.0,0.2391])$ \\
$x_{4}$ & $([0.5159,0.6539],[0.0,0.2730])$ & $([0.4215,0.6386],[0.0,0.2126])$ & $([0.5689,0.6945],[0.0,0.2174])$ \\
$x_{5}$ & $([0.4321,0.6554],[0.1988,0.3172])$ & $([0.4938,0.6837],[0.0,0.2122])$ & $([0.4694,0.7064],[0.0,0.2470])$ \\
\hline
\end{tabular}

TABLE 5: Group decision matrix $\dot{R}$ obtained by utilizing P-GIIFOWA operator.

\begin{tabular}{cccc}
\hline & $d_{1}$ & $d_{2}$ & $d_{3}$ \\
\hline$x_{1}$ & $([0.5951,0.7002],[0.0,0.2500])$ & $([0.4845,0.6879],[0.0,0.2874])$ & $([0.5457,0.6792],[0.2024,0.3094])$ \\
$x_{2}$ & $([0.4667,0.6562],[0.1932,0.3284])$ & $([0.3641,0.6194],[0.1743,0.3104])$ & $([0.4322,0.6338],[0.0,0.2047])$ \\
$x_{3}$ & $([0.4887,0.6132],[0.2058,0.3445])$ & $([0.4322,0.6925],[0.0,0.2048])$ & $([0.4104,0.6071],[0.0,0.2337])$ \\
$x_{4}$ & $([0.5307,0.6741],[0.0,0.2507])$ & $([0.4598,0.6820],[0.0,0.1905])$ & $([0.5970,0.7189],[0.0,0.2175])$ \\
$x_{5}$ & $([0.4486,0.6560],[0.1895,0.3109])$ & $([0.5037,0.6766],[0.0,0.2048])$ & $([0.5006,0.7153],[0.0,0.2344])$ \\
\hline
\end{tabular}

TABLE 6: Group decision matrix $\dot{R}$ obtained by utilizing Gaussian-GIIFOWG operator.

\begin{tabular}{llcr}
\hline & $d_{1}$ & $d_{2}$ & $d_{3}$ \\
\hline$x_{1}$ & $([0.5553,0.6574],[0.1658,0.2805])$ & $([0.4733,0.6588],[0.1677,0.3217])$ & $([0.4555,0.5881],[0.2392,0.3904])$ \\
$x_{2}$ & $([0.4576,0.6285],[0.2247,0.3400])$ & $([0.3387,0.5930],[0.1836,0.3307])$ & $([0.4213,0.6035],[0.1321,0.2279])$ \\
$x_{3}$ & $([0.4732,0.5894],[0.2388,0.3752])$ & $([0.4180,0.6725],[0.1141,0.2302])$ & $([0.3861,0.5724],[0.1463,0.2724])$ \\
$x_{4}$ & $([0.4969,0.6292],[0.1818,0.3117])$ & $([0.3851,0.5905],[0.1202,0.2588])$ & $([0.5400,0.6647],[0.0846,0.2217])$ \\
$x_{5}$ & $([0.4104,0.6345],[0.2129,0.3299])$ & $([0.4562,0.6658],[0.0972,0.2302])$ & $([0.4351,0.6871],[0.1329,0.2719])$ \\
\hline
\end{tabular}


TABLE 7: Group decision matrix $\dot{R}$ obtained by utilizing P-GIIFOWG operator.

\begin{tabular}{llcr}
\hline & $d_{1}$ & $d_{2}$ & $d_{3}$ \\
\hline$x_{1}$ & $([0.5669,0.6689],[0.1473,0.2655])$ & $([0.4735,0.6745],[0.1663,0.3070])$ & $([0.4247,0.5680],[0.2473,0.4063])$ \\
$x_{2}$ & $([0.4537,0.6317],[0.2258,0.3443])$ & $([0.3506,0.5913],[0.1811,0.3239])$ & $([0.3927,0.5645],[0.1240,0.2235])$ \\
$x_{3}$ & $([0.4687,0.5886],[0.2245,0.3684])$ & $([0.4011,0.6443],[0.1042,0.2234])$ & $([0.3819,0.5663],[0.1424,0.2661])$ \\
$x_{4}$ & $([0.5105,0.6503],[0.1671,0.2907])$ & $([0.4134,0.6202],[0.1060,0.2312])$ & $([0.5693,0.6926],[0.0810,0.2218])$ \\
$x_{5}$ & $([0.4270,0.6319],[0.2032,0.3244])$ & $([0.4592,0.6535],[0.0838,0.2234])$ & $([0.4636,0.6959],[0.1244,0.2662)$ \\
\hline
\end{tabular}

TABLE 8: Overall group decision assessment values for all alternatives.

\begin{tabular}{lccccc}
\hline $\begin{array}{l}\text { Combination of } \\
\text { operators }\end{array}$ & $x_{1}$ & $x_{2}$ & $x_{3}$ & $x_{4}$ & $x_{5}$ \\
\hline Gaussian-GIIFOWA & $([0.5481,0.6859]$, & $([0.4322,0.6491]$, & $([0.4437,0.6427]$, & $([0.5125,0.6664]$, & $([0.4661,0.6850]$, \\
and IIFWA & $[0.0,0.2877])$ & $[0.0,0.2718])$ & $[0.0,0.2597])$ & $[0.0,0.2312])$ & $[0.0,0.2544])$ \\
\hline P-GIIFOWA and & $([0.5442,0.6882]$, & $([0.4235,0.6365]$, & $([0.4414,0.6367]$, & $([0.5394,0.6951]$, & $([0.4865,0.6869]$, \\
IIFWA & $[0.0,0.2839])$ & $[0.0,0.2673])$ & $[0.0,0.2524])$ & $[0.0,0.2181])$ & $[0.0,0.2450])$ \\
\hline Gaussian-GIIFOWG & $([0.4890,0.6292]$, & $([0.4045,0.6077]$, & $([0.4203,0.6061]$, & $([0.4759,0.6310]$, & $([0.4337,0.6646]$, \\
and IIFWG & $[0.1965,0.3385])$ & $[0.1762,0.2943])$ & $[0.1660,0.2930])$ & $[0.1254,0.2608])$ & $[0.1475,0.2778])$ \\
\hline P-GIIFOWG and & $([0.4785,0.6281]$, & $([0.3964,0.5921]$, & $([0.4121,0.5955]$, & $([0.5005,0.6575]$, & $([0.4510,0.6634]$, \\
IIFWG & $[0.1943,0.3371])$ & $[0.1728,0.2919])$ & $[0.1570,0.2864])$ & $[0.1151,0.2459])$ & $[0.1372,0.2719])$ \\
\hline
\end{tabular}

TABLE 9: Orderings of the alternatives obtained by using different operators.

\begin{tabular}{lc}
\hline Different combination of operators & \multicolumn{1}{c}{ Ordering } \\
\hline Gaussian-GIIFOWA and IIFWA & $x_{2} \prec x_{3} \prec x_{5} \prec x_{1} \prec x_{4}$ \\
P-GIIFOWA and IIFWA & $x_{2} \prec x_{3} \prec x_{5} \prec x_{1} \prec x_{4}$ \\
Gaussian-GIIFOWG and IIFWG & $x_{2} \prec x_{3} \prec x_{1} \prec x_{5} \prec x_{4}$ \\
P-GIIFOWG and IIFWG & $x_{2} \prec x_{3} \prec x_{1} \prec x_{5} \prec x_{4}$ \\
\hline
\end{tabular}

fuzzy overall group decision assessment values for all alternatives. The results are shown in Table 8.

Step 5. Calculate the scores $S\left(\widetilde{r}_{i}\right)(i=1,2,3,4,5)$ of the group overall intuitionistic fuzzy assessment values and rank all alternatives in accordance with scores $S\left(\widetilde{r}_{i}\right)$; the obtained ordering results are listed in Table 9.

As can be seen from Table 9, for all four combinations of operators, alternative $x_{4}$ is consistently distinguished as the best one, and alternative $x_{2}$ and $x_{3}$ are consistently distinguished as the worst ones. The ordering of $x_{1}$ and $x_{5}$ shows difference with IIFWA or IIFWG adopted. The first two combinations of averaging operators yield the same ranking result as $x_{2} \prec x_{3} \prec x_{5} \prec x_{1} \prec x_{4}$ and the last two combinations of geometric operators also generate the same ranking result as $x_{2} \prec x_{3} \prec x_{1} \prec x_{5} \prec x_{4}$, which show that the proposed Gaussian distribution-based operators and power method-based operators can help to effectively differentiate the most desirable one(s). Generally, from the aspect of different support degree measurement methods adopted, the Gaussian-GIIFOWA operator and Gaussian-GIIFOWG operator appear to be more straight and concise than the $\mathrm{P}$ GIIFOWA operator and P-GIIFOWG operator, while the latter two operators can utilize preference more completely by considering not only support degree of each argument by other arguments but also the support degree between the aggregated argument and the mean value. So for different practical decision making problems, decision makers may choose different operators according to their preference and the related facts.

5.2. Further Discussion. In order to further verify properties of the proposed four generalized argument-dependent aggregation operators, experiments are conducted in this subsection with attitudinal parameter $\lambda$ varying in a crisp range: $1 / 5,1 / 4,1 / 3,1 / 2,1,2,3,4$, and 5 . For clarity, the proposed Gaussian-GIIFOWA operator, Gaussian-GIIFOWG operator, P-GIIFOWA operator, and P-GIIFOWG operator are, respectively, applied on assessment matrix given by decision maker $d_{1}$ (as shown in Table 4 ), and corresponding results are listed in Table 10 to Table 13.

From comparison with the last columns of Table 10 to Table 13, it is can be seen that the best and worst alternatives are totally consistent and only the orderings of $x_{2}$ and $x_{5}$ exhibit some difference, which shows that all the proposed four aggregation operators can effectively distinguish the most desirable alternatives. And from the view of results obtained by Gaussian-GIIFOWA and Gaussian-GIIFOWG with ranging $\lambda$, it is can be observed that all the score values in Table 11 are smaller than the score values in Table 10 with $\lambda=$ 1 (Gaussian-GIIFOWA is reduced to be Gaussian-IIFOWA), and that all the score values in Table 10 are bigger than the score values in Table 11 with $\lambda=1$ (Gaussian-GIIFOWG reduces to Gaussian-IIFOWG). These observed facts just verify the validness of the inequations given in Theorem 20 . And similarly, the same facts verifying the validness of Theorem 28 can also be observed by comparing the score values listed in Tables 12 and 13. 
TABLE 10: Ranking results obtained by applying Gaussian-GIIFOWA on assessments given by $d_{1}$.

\begin{tabular}{|c|c|c|c|}
\hline Aggregation operator & $\lambda$ & Score values & Ranking \\
\hline \multirow{9}{*}{ Gaussian-GIIFOWA } & $1 / 5$ & $s\left(\widetilde{r}_{1}\right)=0.9965, s\left(\widetilde{r}_{2}\right)=0.6022, s\left(\widetilde{r}_{3}\right)=0.5121, s\left(\widetilde{r}_{4}\right)=0.8839, s\left(\widetilde{r}_{5}\right)=0.5594$ & $x_{1}>x_{4}>x_{2}>x_{5}>x_{3}$ \\
\hline & $1 / 4$ & $s\left(\widetilde{r}_{1}\right)=0.9972, s\left(\widetilde{r}_{2}\right)=0.6030, s\left(\widetilde{r}_{3}\right)=0.5128, s\left(\widetilde{r}_{4}\right)=0.8874, s\left(\widetilde{r}_{5}\right)=0.5601$ & $x_{1}>x_{4}>x_{2}>x_{5}>x_{3}$ \\
\hline & $1 / 3$ & $s\left(\widetilde{r}_{1}\right)=0.9984, s\left(\widetilde{r}_{2}\right)=0.6044, s\left(\widetilde{r}_{3}\right)=0.5141, s\left(\widetilde{r}_{4}\right)=0.8860, s\left(\widetilde{r}_{5}\right)=0.5613$ & $x_{1}>x_{4}>x_{2}>x_{5}>x_{3}$ \\
\hline & $1 / 2$ & $s\left(\widetilde{r}_{1}\right)=1.008, s\left(\widetilde{r}_{2}\right)=0.6071, s\left(\widetilde{r}_{3}\right)=0.5167, s\left(\widetilde{r}_{4}\right)=0.8886, s\left(\widetilde{r}_{5}\right)=0.5638$ & $x_{1}>x_{4}>x_{2}>x_{5}>x_{3}$ \\
\hline & 1 & $s\left(\widetilde{r}_{1}\right)=1.008, s\left(\widetilde{r}_{2}\right)=0.6156, s\left(\widetilde{r}_{3}\right)=0.5245, s\left(\widetilde{r}_{4}\right)=0.8968, s\left(\widetilde{r}_{5}\right)=0.5716$ & $x_{1}>x_{4}>x_{2}>x_{5}>x_{3}$ \\
\hline & 2 & $s\left(\widetilde{r}_{1}\right)=1.0231, s\left(\widetilde{r}_{2}\right)=0.6341, s\left(\widetilde{r}_{3}\right)=0.541, s\left(\widetilde{r}_{4}\right)=0.9147, s\left(\widetilde{r}_{5}\right)=0.5887$ & $x_{1}>x_{4}>x_{2}>x_{5}>x_{3}$ \\
\hline & 3 & $s\left(\widetilde{r}_{1}\right)=1.0386, s\left(\widetilde{r}_{2}\right)=0.6542, s\left(\widetilde{r}_{3}\right)=0.558, s\left(\widetilde{r}_{4}\right)=0.9343, s\left(\widetilde{r}_{5}\right)=0.6075$ & $x_{1}>x_{4}>x_{2}>x_{5}>x_{3}$ \\
\hline & 4 & $s\left(\widetilde{r}_{1}\right)=1.054, s\left(\widetilde{r}_{2}\right)=0.6755, s\left(\widetilde{r}_{3}\right)=0.575, s\left(\widetilde{r}_{4}\right)=0.9552, s\left(\widetilde{r}_{5}\right)=0.6272$ & $x_{1}>x_{4}>x_{2}>x_{5}>x_{3}$ \\
\hline & 5 & $s\left(\widetilde{r}_{1}\right)=1.0688, s\left(\widetilde{r}_{2}\right)=0.6972, s\left(\widetilde{r}_{3}\right)=0.5917, s\left(\widetilde{r}_{4}\right)=0.9767, s\left(\widetilde{r}_{5}\right)=0.6471$ & $x_{1}>x_{4}>x_{2}>x_{5}>x_{3}$ \\
\hline
\end{tabular}

TABLE 11: Ranking results obtained by applying Gaussian-GIIFOWG on assessments given by $d_{1}$.

\begin{tabular}{|c|c|c|c|}
\hline Aggregation operator & $\lambda$ & Score value & Ranking \\
\hline \multirow{9}{*}{ Gaussian-GIIFOWG } & $1 / 5$ & $s\left(\widetilde{r}_{1}\right)=0.841, s\left(\widetilde{r}_{2}\right)=0.5523, s\left(\widetilde{r}_{3}\right)=0.4714, s\left(\widetilde{r}_{4}\right)=0.7077, s\left(\widetilde{r}_{5}\right)=0.5225$ & $x_{1}>x_{4}>x_{2}>x_{5}>x_{3}$ \\
\hline & $1 / 4$ & $s\left(\widetilde{r}_{1}\right)=0.8327, s\left(\widetilde{r}_{2}\right)=0.5505, s\left(\widetilde{r}_{3}\right)=0.4701, s\left(\widetilde{r}_{4}\right)=0.699, s\left(\widetilde{r}_{5}\right)=0.5213$ & $x_{1}>x_{4}>x_{2}>x_{5}>x_{3}$ \\
\hline & $1 / 3$ & $s\left(\widetilde{r}_{1}\right)=0.8215, s\left(\widetilde{r}_{2}\right)=0.5475, s\left(\widetilde{r}_{3}\right)=0.4678, s\left(\widetilde{r}_{4}\right)=0.6873, s\left(\widetilde{r}_{5}\right)=0.5193$ & $x_{1}>x_{4}>x_{2}>x_{5}>x_{3}$ \\
\hline & $1 / 2$ & $s\left(\widetilde{r}_{1}\right)=0.8041, s\left(\widetilde{r}_{2}\right)=0.5413, s\left(\widetilde{r}_{3}\right)=0.4632, s\left(\widetilde{r}_{4}\right)=0.6694, s\left(\widetilde{r}_{5}\right)=0.5152$ & $x_{1}>x_{4}>x_{2}>x_{5}>x_{3}$ \\
\hline & 1 & $s\left(\widetilde{r}_{1}\right)=0.7664, s\left(\widetilde{r}_{2}\right)=0.5215, s\left(\widetilde{r}_{3}\right)=0.4486, s\left(\widetilde{r}_{4}\right)=0.6325, s\left(\widetilde{r}_{5}\right)=0.5022$ & $x_{1}>x_{4}>x_{2}>x_{5}>x_{3}$ \\
\hline & 2 & $s\left(\widetilde{r}_{1}\right)=0.7059, s\left(\widetilde{r}_{2}\right)=0.4811, s\left(\widetilde{r}_{3}\right)=0.4168, s\left(\widetilde{r}_{4}\right)=0.5795, s\left(\widetilde{r}_{5}\right)=0.4741$ & $x_{1}>x_{4}>x_{2}>x_{5}>x_{3}$ \\
\hline & 3 & $s\left(\widetilde{r}_{1}\right)=0.6513, s\left(\widetilde{r}_{2}\right)=0.4449, s\left(\widetilde{r}_{3}\right)=0.3838, s\left(\widetilde{r}_{4}\right)=0.5371, s\left(\widetilde{r}_{5}\right)=0.4459$ & $x_{1}>x_{4}>x_{2}>x_{5}>x_{3}$ \\
\hline & 4 & $s\left(\widetilde{r}_{1}\right)=0.6024, s\left(\widetilde{r}_{2}\right)=0.4149, s\left(\widetilde{r}_{3}\right)=0.3513, s\left(\widetilde{r}_{4}\right)=0.5019, s\left(\widetilde{r}_{5}\right)=0.4194$ & $x_{1}>x_{4}>x_{5}>x_{2}>x_{3}$ \\
\hline & 5 & $s\left(\widetilde{r}_{1}\right)=0.56, s\left(\widetilde{r}_{2}\right)=0.3905, s\left(\widetilde{r}_{3}\right)=0.3199, s\left(\widetilde{r}_{4}\right)=0.4725, s\left(\widetilde{r}_{5}\right)=0.3952$ & $x_{1}>x_{4}>x_{5}>x_{2}>x_{3}$ \\
\hline
\end{tabular}

TABLE 12: Ranking results obtained by applying P-GIIFOWA on assessments given by $d_{1}$.

\begin{tabular}{|c|c|c|c|}
\hline Aggregation operator & $\lambda$ & Score value & Ranking \\
\hline \multirow{9}{*}{ P-GIIFOWA } & $1 / 5$ & $s\left(\widetilde{r}_{1}\right)=1.0344, s\left(\widetilde{r}_{2}\right)=0.5892, s\left(\widetilde{r}_{3}\right)=0.5375, s\left(\widetilde{r}_{4}\right)=0.9417, s\left(\widetilde{r}_{5}\right)=0.5918$ & $x_{1}>x_{4}>x_{5}>x_{2}>x_{3}$ \\
\hline & $1 / 4$ & $s\left(\widetilde{r}_{1}\right)=1.035, s\left(\widetilde{r}_{2}\right)=0.5899, s\left(\widetilde{r}_{3}\right)=0.5383, s\left(\widetilde{r}_{4}\right)=0.9424, s\left(\widetilde{r}_{5}\right)=0.5925$ & $x_{1}>x_{4}>x_{5}>x_{2}>x_{3}$ \\
\hline & $1 / 3$ & $s\left(\widetilde{r}_{1}\right)=1.0361, s\left(\widetilde{r}_{2}\right)=0.5911, s\left(\widetilde{r}_{3}\right)=0.5398, s\left(\widetilde{r}_{4}\right)=0.9437, s\left(\widetilde{r}_{5}\right)=0.5938$ & $x_{1}>x_{4}>x_{5}>x_{2}>x_{3}$ \\
\hline & $1 / 2$ & $s\left(\widetilde{r}_{1}\right)=1.0384, s\left(\widetilde{r}_{2}\right)=0.5936, s\left(\widetilde{r}_{3}\right)=0.5427, s\left(\widetilde{r}_{4}\right)=0.9462, s\left(\widetilde{r}_{5}\right)=0.5963$ & $x_{1}>x_{4}>x_{5}>x_{2}>x_{3}$ \\
\hline & 1 & $s\left(\widetilde{r}_{1}\right)=1.0453, s\left(\widetilde{r}_{2}\right)=0.6013, s\left(\widetilde{r}_{3}\right)=0.5517, s\left(\widetilde{r}_{4}\right)=0.954, s\left(\widetilde{r}_{5}\right)=0.6042$ & $x_{1}>x_{4}>x_{5}>x_{2}>x_{3}$ \\
\hline & 2 & $s\left(\widetilde{r}_{1}\right)=1.0595, s\left(\widetilde{r}_{2}\right)=0.6182, s\left(\widetilde{r}_{3}\right)=0.5704, s\left(\widetilde{r}_{4}\right)=0.9708, s\left(\widetilde{r}_{5}\right)=0.6214$ & $x_{1}>x_{4}>x_{5}>x_{2}>x_{3}$ \\
\hline & 3 & $s\left(\widetilde{r}_{1}\right)=1.0741, s\left(\widetilde{r}_{2}\right)=0.6367, s\left(\widetilde{r}_{3}\right)=0.5895, s\left(\widetilde{r}_{4}\right)=0.989, s\left(\widetilde{r}_{5}\right)=0.64$ & $x_{1}>x_{4}>x_{5}>x_{2}>x_{3}$ \\
\hline & 4 & $s\left(\widetilde{r}_{1}\right)=1.0884, s\left(\widetilde{r}_{2}\right)=0.6564, s\left(\widetilde{r}_{3}\right)=0.6083, s\left(\widetilde{r}_{4}\right)=1.0081, s\left(\widetilde{r}_{5}\right)=0.6594$ & $x_{1}>x_{4}>x_{5}>x_{2}>x_{3}$ \\
\hline & 5 & $s\left(\widetilde{r}_{1}\right)=1.1021, s\left(\widetilde{r}_{2}\right)=0.6767, s\left(\widetilde{r}_{3}\right)=0.6263, s\left(\widetilde{r}_{4}\right)=1.0275, s\left(\widetilde{r}_{5}\right)=0.6788$ & $x_{1}>x_{4}>x_{5}>x_{2}>x_{3}$ \\
\hline
\end{tabular}


TABLE 13: Ranking results obtained by applying P-GIIFOWG on assessments given by $d_{1}$.

\begin{tabular}{|c|c|c|c|}
\hline Aggregation operator & $\lambda$ & Score value & Ranking \\
\hline \multirow{9}{*}{ P-GIIFOWG } & $1 / 5$ & $s\left(\widetilde{r}_{1}\right)=0.9026, s\left(\widetilde{r}_{2}\right)=0.5437, s\left(\widetilde{r}_{3}\right)=0.4907, s\left(\widetilde{r}_{4}\right)=0.7869, s\left(\widetilde{r}_{5}\right)=0.5531$ & $x_{1}>x_{4}>x_{5}>x_{2}>x_{3}$ \\
\hline & $1 / 4$ & $s\left(\widetilde{r}_{1}\right)=0.8938, s\left(\widetilde{r}_{2}\right)=0.542, s\left(\widetilde{r}_{3}\right)=0.4892, s\left(\widetilde{r}_{4}\right)=0.7773, s\left(\widetilde{r}_{5}\right)=0.5518$ & $x_{1}>x_{4}>x_{5}>x_{2}>x_{3}$ \\
\hline & $1 / 3$ & $s\left(\widetilde{r}_{1}\right)=0.8818, s\left(\widetilde{r}_{2}\right)=0.5392, s\left(\widetilde{r}_{3}\right)=0.4867, s\left(\widetilde{r}_{4}\right)=0.7642, s\left(\widetilde{r}_{5}\right)=0.5497$ & $x_{1}>x_{4}>x_{5}>x_{2}>x_{3}$ \\
\hline & $1 / 2$ & $s\left(\widetilde{r}_{1}\right)=0.8631, s\left(\widetilde{r}_{2}\right)=0.5335, s\left(\widetilde{r}_{3}\right)=0.4814, s\left(\widetilde{r}_{4}\right)=0.7442, s\left(\widetilde{r}_{5}\right)=0.5453$ & $x_{1}>x_{4}>x_{5}>x_{2}>x_{3}$ \\
\hline & 1 & $s\left(\widetilde{r}_{1}\right)=0.8229, s\left(\widetilde{r}_{2}\right)=0.5154, s\left(\widetilde{r}_{3}\right)=0.4644, s\left(\widetilde{r}_{4}\right)=0.7029, s\left(\widetilde{r}_{5}\right)=0.5313$ & $x_{1}>x_{4}>x_{5}>x_{2}>x_{3}$ \\
\hline & 2 & $s\left(\widetilde{r}_{1}\right)=0.7581, s\left(\widetilde{r}_{2}\right)=0.4783, s\left(\widetilde{r}_{3}\right)=0.4271, s\left(\widetilde{r}_{4}\right)=0.6435, s\left(\widetilde{r}_{5}\right)=0.5012$ & $x_{1}>x_{4}>x_{2}>x_{5}>x_{3}$ \\
\hline & 3 & $s\left(\widetilde{r}_{1}\right)=0.6987, s\left(\widetilde{r}_{2}\right)=0.4451, s\left(\widetilde{r}_{3}\right)=0.3884, s\left(\widetilde{r}_{4}\right)=0.5956, s\left(\widetilde{r}_{5}\right)=0.4709$ & $x_{1}>x_{4}>x_{5}>x_{2}>x_{3}$ \\
\hline & 4 & $s\left(\widetilde{r}_{1}\right)=0.6453, s\left(\widetilde{r}_{2}\right)=0.4176, s\left(\widetilde{r}_{3}\right)=0.3505, s\left(\widetilde{r}_{4}\right)=0.5555, s\left(\widetilde{r}_{5}\right)=0.4424$ & $x_{1}>x_{4}>x_{5}>x_{2}>x_{3}$ \\
\hline & 5 & $s\left(\widetilde{r}_{1}\right)=0.5987, s\left(\widetilde{r}_{2}\right)=0.395, s\left(\widetilde{r}_{3}\right)=0.3146, s\left(\widetilde{r}_{4}\right)=0.5217, s\left(\widetilde{r}_{5}\right)=0.4164$ & $x_{1}>x_{4}>x_{5}>x_{2}>x_{3}$ \\
\hline
\end{tabular}

In summary, the proposed four generalized argumentdependent operators can effectively and objectively distinguish the most desirable alternative(s). The Gaussian weighting vector directly measures support degree of arguments with less computation complexity by only considering the distance between arguments with mean value, while the power weighting vector obtained by hybrid support function can consider objective preference information by measuring both the support degrees between arguments and the support degrees between arguments and mid values. In practical decision modelling process, decision makers can select suitably presented operators and proper parameter $\lambda$ in accordance with attitudinal preference or problem characteristics to help effectively solve multiple attribute group decision making problems under interval-valued intuitionistic fuzzy environments.

\section{Conclusions}

We have investigated some generalized argument-dependent aggregation operators for MAGDM under interval-valued intuitionistic fuzzy environments. First, a Gaussian distribution-based method for deriving weighting vector by measuring distances between arguments and mean value has been presented, based on which we have proposed the GaussianGIIFOWA operator and the Gaussian-GIIFOWG operator. Then, a hybrid support degree function has been devised for measuring both the support degrees between arguments and the support degrees between arguments and mean value, based on which we have also proposed the P-GIIFOWA operator and the P-GIIFOWG operator. And some desirable properties of the proposed dependent operators have been analyzed. The main advantages of developed operators are that they can relieve the influence of unfair assessments on the results by assigning low weights to those false and biased ones, and they can include a wide range of other aggregation operators for decision makers to flexibly choose in practical decision modelling. An approach has been formed based on developed operators and applied to solve the MAGDM problem concerning exploitation investment evaluation of tourist spots for verifying effectiveness and practicality of proposed methods. Furthermore, the results of comparative experiments have also verified the properties of developed operators. In the future, we will continue working on the extension and application of these operators to other fields under different environments, such as the information fusion, data mining, and hybrid decision making indices with hesitant fuzzy preference.

\section{Acknowledgments}

This work was supported in part by the National Science Foundation of China (no. 71201145, no. 71271072), the Research Fund for the Doctoral Program of Higher Education of China (no. 20110111110006), the Social Science Foundation of Ministry of Education of China (no. 11YJC630283), and Zhejiang Provincial Natural Science Foundation of China (no. Y6110345).

\section{References}

[1] M. Z. Zerafat Angiz, A. Emrouznejad, A. Mustafa, and A. Rashidi Komijan, "Selecting the most preferable alternatives in a group decision making problem using DEA," Expert Systems with Applications, vol. 36, no. 5, pp. 9599-9602, 2009.

[2] Y. C. Dong, G. Q. Zhang, W. C. Hong, and Y. F. Xu, "Consensus models for AHP group decision making under row geometric mean prioritization method," Decision Support Systems, vol. 49, no. 3, pp. 281-289, 2010.

[3] L. A. Yu and K. K. Lai, "A distance-based group decision-making methodology for multi-person multi-criteria emergency decision support," Decision Support Systems, vol. 51, no. 2, pp. 307315, 2011.

[4] L. G. Zhou, H. Y. Chen, and J. P. Liu, "Generalized weighted exponential proportional aggregation operators and their applications to group decision making," Applied Mathematical Modelling, vol. 36, no. 9, pp. 4365-4384, 2012.

[5] L. G. Zhou, H. Y. Chen, and J. P. Liu, "Generalized logarithmic proportional averaging operators and their applications to 
group decision making," Knowledge-Based Systems, vol. 36, pp. 268-279, 2012.

[6] Q.-W. Cao and J. Wu, "The extended COWG operators and their application to multiple attributive group decision making problems with interval numbers," Applied Mathematical Modelling, vol. 35, no. 5, pp. 2075-2086, 2011.

[7] L. G. Zhou, H. Y. Chen, J. M. Merigó, and A. M. Gil-Lafuente, "Uncertain generalized aggregation operators," Expert Systems with Applications, vol. 39, pp. 1105-1117, 2012.

[8] L.-G. Zhou and H.-Y. Chen, "Continuous generalized OWA operator and its application to decision making," Fuzzy Sets and Systems, vol. 168, pp. 18-34, 2011.

[9] L. G. Zhou and H. Y. Chen, "A generalization of the power aggregation operators for linguistic environment and its application in group decision making," Knowledge-Based Systems, vol. 26, pp. 216-224, 2012.

[10] J. M. Merigó, A. M. Gil-Lafuente, L. G. Zhou, and H. Y. Chen, "Induced and linguistic generalized aggregation operators and their application in linguistic group decision making," Group Decision and Negotiation, vol. 21, pp. 531-549, 2010.

[11] G. W. Wei and X. F. Zhao, "Some dependent aggregation operators with 2-tuple linguistic information and their application to multiple attribute group decision making," Expert Systems with Applications, vol. 39, pp. 5881-5886, 2012.

[12] G. W. Wei, "FIOWHM operator and its application to multiple attribute group decision making," Expert Systems with Applications, vol. 38, no. 4, pp. 2984-2989, 2011.

[13] J. M. Merigó and A. M. Gil-Lafuente, "Fuzzy induced generalized aggregation operators and its application in multi-person decision making," Expert Systems with Applications, vol. 38, no. 8, pp. 9761-9772, 2011.

[14] P. D. Liu, "A weighted aggregation operators multi-attribute group decision-making method based on interval-valued trapezoidal fuzzy numbers," Expert Systems with Applications, vol. 38, no. 1, pp. 1053-1060, 2011.

[15] F. Zandi and M. Tavana, "A fuzzy group multi-criteria enterprise architecture framework selection model," Expert Systems with Applications, vol. 39, pp. 1165-1173, 2012.

[16] K. Khalili-Damghani and S. Sadi-Nezhad, "A hybrid fuzzy multiple criteria group decision making approach for sustainable project selection," Applied Soft Computing, vol. 13, pp. 339-352, 2013.

[17] B. Vahdani, S. M. Mousavi, H. Hashemi, M. Mousakhani, and R. Tavakkoli-Moghaddam, "A new compromise solution method for fuzzy group decision-making problems with an application to the contractor selection," Engineering Applications of Artificial Intelligence, vol. 26, pp. 779-788, 2013.

[18] K. T. Atanassov, "Intuitionistic fuzzy sets," Fuzzy Sets and Systems, vol. 20, no. 1, pp. 87-96, 1986.

[19] K. Atanassov and G. Gargov, "Interval valued intuitionistic fuzzy sets," Fuzzy Sets and Systems, vol. 31, no. 3, pp. 343-349, 1989.

[20] D.-F. Li, G.-H. Chen, and Z.-G. Huang, "Linear programming method for multiattribute group decision making using IF sets," Information Sciences, vol. 180, no. 9, pp. 1591-1609, 2010.

[21] Z. X. Su, G. P. Xia, M. Y. Chen, and L. Wang, "Induced generalized intuitionistic fuzzy OWA operator for multi-attribute group decision making," Expert Systems with Applications, vol. 39, pp. 1902-1910, 2012.

[22] Y. J. Xu and H. M. Wang, "The induced generalized aggregation operators for intuitionistic fuzzy sets and their application in group decision making," Applied Soft Computing, vol. 12, pp. 1168-1179, 2012.
[23] L. G. Zhou, H. Y. Chen, and J. P. Liu, "Generalized power aggregation operators and their applications in group decision making," Computers \& Industrial Engineering, vol. 62, pp. 989-999, 2012.

[24] Z. S. Xu, "Approaches to multiple attribute group decision making based on intuitionistic fuzzy power aggregation operators," Knowledge-Based Systems, vol. 24, no. 6, pp. 749-760, 2011.

[25] Z. P. Chen and W. Yang, "A new multiple attribute group decision making method in intuitionistic fuzzy setting," Applied Mathematical Modelling, vol. 35, no. 9, pp. 4424-4437, 2011.

[26] Z. X. Su, M. Y. Chen, G. P. Xia, and L. Wang, "An interactive method for dynamic intuitionistic fuzzy multi-attribute group decision making," Expert Systems with Applications, vol. 38, pp. 15286-15295, 2011.

[27] S. P. Wan, "Power average operators of trapezoidal intuitionistic fuzzy numbers and application to multi-attribute group decision making," Applied Mathematical Modelling, vol. 37, pp. 41124126, 2013.

[28] Z. L. Yue and Y. Y. Jia, "An application of soft computing technique in group decision making under interval-valued intuitionistic fuzzy environment," Applied Soft Computing, vol. 13, pp. 2490-2503, 2013.

[29] D. J. Yu, Y. Y. Wu, and T. Lu, "Interval-valued intuitionistic fuzzy prioritized operators and their application in group decision making," Knowledge-Based Systems, vol. 30, pp. 57-66, 2012.

[30] M. M. Xia and Z. S. Xu, "Entropy/cross entropy-based group decision making under intuitionistic fuzzy environment," Information Fusion, vol. 13, pp. 31-47, 2012.

[31] Y. Jiang, Z. S. Xu, and X. H. Yu, "Compatibility measures and consensus models for group decision making with intuitionistic multiplicative preference relations," Applied Soft Computing, vol. 13, pp. 2075-2086, 2013.

[32] R. R. Yager, "On ordered weighted averaging aggregation operators in multicriteria decisionmaking," IEEE Transactions on Systems, Man, and Cybernetics, vol. 18, no. 1, pp. 183-190, 1988.

[33] J. M. Merigó and M. Casanovas, "Decision-making with distance measures and induced aggregation operators," Computers and Industrial Engineering, vol. 60, no. 1, pp. 66-76, 2011.

[34] G. W. Wei, "A method for multiple attribute group decision making based on the ET-WG and ET-OWG operators with 2tuple linguistic information," Expert Systems with Applications, vol. 37, no. 12, pp. 7895-7900, 2010.

[35] N. B. Karayiannis, "Soft learning vector quantization and clustering algorithms based on ordered weighted aggregation operators," IEEE Transactions on Neural Networks, vol. 11, no. 5, pp. 1093-1105, 2000.

[36] D. F. Li, "Multiattribute decision making method based on generalized OWA operators with intuitionistic fuzzy sets," Expert Systems with Applications, vol. 37, no. 12, pp. 8673-8678, 2010.

[37] J. M. Merigó and M. Casanovas, "Fuzzy generalized hybrid aggregation operators and its application in fuzzy decision making," International Journal of Fuzzy Systems, vol. 12, no. 1, pp. $15-24,2010$.

[38] Y. J. Xu, J. M. Merigó, and H. M. Wang, "Linguistic power aggregation operators and their application to multiple attribute group decision making," Applied Mathematical Modelling, vol. 36, no. 11, pp. 5427-5444, 2012.

[39] Z. S. Xu and R. R. Yager, "Power-geometric operators and their use in group decision making," IEEE Transactions on Fuzzy Systems, vol. 18, no. 1, pp. 94-105, 2010. 
[40] P. D. Liu, "Some generalized dependent aggregation operators with intuitionistic linguistic numbers and their application to group decision making," Journal of Computer and System Sciences, vol. 79, no. 1, pp. 131-143, 2013.

[41] Z. S. Xu, "Dependent OWA operators," Lecture Notes in Computer Science, vol. 3885, pp. 172-178, 2006.

[42] D. P. Filev and R. R. Yager, "On the issue of obtaining OWA operator weights," Fuzzy Sets and Systems, vol. 94, no. 2, pp. 157169, 1998.

[43] R. Fullér and P. Majlender, "An analytic approach for obtaining maximal entropy OWA operator weights," Fuzzy Sets and Systems, vol. 124, no. 1, pp. 53-57, 2001.

[44] Z. S. Xu, "An overview of methods for determining OWA weights," International Journal of Intelligent Systems, vol. 20, no. 8, pp. 843-865, 2005.

[45] R. R. Yager, "Families of OWA operators," Fuzzy Sets and Systems, vol. 59, no. 2, pp. 125-148, 1993.

[46] R. R. Yager and D. P. Filev, "Parametrized and-like and or-like own operators," International Journal of General Systems, vol. 22, no. 3, pp. 297-316, 1994.

[47] P. D. Liu and F. Jin, "Methods for aggregating intuitionistic uncertain linguistic variables and their application to group decision making," Information Sciences, vol. 205, pp. 58-71, 2012.

[48] P. D. Liu, "Some geometric aggregation operators based on interval intuitionistic uncertain linguistic variables and their application to group decision making," Applied Mathematical Modelling, vol. 37, no. 4, pp. 2430-2444, 2013.

[49] Z. S. Xu, "Dependent uncertain ordered weighted aggregation operators," Information Fusion, vol. 9, no. 2, pp. 310-316, 2008.

[50] Z. S. Xu and Q. L. Da, “The uncertain OWA operator," International Journal of Intelligent Systems, vol. 17, no. 6, pp. 569-575, 2002.

[51] G. W. Wei, "Method of uncertain linguistic multiple attribute group decision making based on dependent aggregation operators," Systems Engineering and Electronics, vol. 32, no. 4, pp. 764769, 2010.

[52] J. Wu, C. Y. Liang, and Y. Q. Huang, "An argument-dependent approach to determining OWA operator weights based on the rule of maximum entropy," International Journal of Intelligent Systems, vol. 22, no. 2, pp. 209-221, 2007.

[53] Z. J. Wang, K. W. Li, and W. Z. Wang, "An approach to multiattribute decision making with interval-valued intuitionistic fuzzy assessments and incomplete weights," Information Sciences, vol. 179, no. 17, pp. 3026-3040, 2009.

[54] R. R. Yager, “The power average operator," IEEE Transactions on Systems, Man, and Cybernetics Part A, vol. 31, no. 6, pp. 724-731, 2001.

[55] Z. S. Xu and M. M. Xia, "Induced generalized intuitionistic fuzzy operators," Knowledge-Based Systems, vol. 24, no. 2, pp. 197-209, 2011.

[56] R. R. Yager, “Generalized OWA aggregation operators," Fuzzy Optimization and Decision Making, vol. 3, no. 1, pp. 93-107, 2004.

[57] J. Fofor, J. L. Marichal, and M. Roubens, "Characterization of the ordered weighted averaging operators," IEEE Transactions on Fuzzy Systems, vol. 3, pp. 236-240, 1995.

[58] J. M. Merigó and A. M. Gil-Lafuente, “The induced generalized OWA operator," Information Sciences, vol. 179, no. 6, pp. 729741, 2009.
[59] R. R. Yager and D. P. Filev, "Induced ordered weighted averaging operators," IEEE Transactions on Systems, Man, and Cybernetics Part B, vol. 29, no. 2, pp. 141-150, 1999.

[60] J. M. Merigó and M. Casanovas, “The generalized hybrid averaging operator and its application in decision making," Journal of Quantitative Methods For Economics and Business Administration, vol. 9, pp. 69-84, 2010.

[61] J. M. Merigó and A. M. Gil-Lafuente, "The induced linguistic generalized OWA operator," in Proceedings of the FLINS International Conference, pp. 325-330, Madrid, Spain, 2008.

[62] H. Zhao, Z. S. Xu, M. F. Ni, and S. Liu, "Generalized aggregation operators for intuitionistic fuzzy sets," International Journal of Intelligent Systems, vol. 25, no. 1, pp. 1-30, 2010.

[63] G. W. Wei, "Some induced geometric aggregation operators with intuitionistic fuzzy information and their application to group decision making," Applied Soft Computing, vol. 10, no. 2, pp. 423-431, 2010.

[64] Y. J. Xu and H. M. Wang, "Approaches based on 2-tuple linguistic power aggregation operators for multiple attribute group decision making under linguistic environment," Applied Soft Computing, vol. 11, no. 5, pp. 3988-3997, 2011.

[65] Z. S. Xu, "Methods for aggregating interval-valued intuitionistic fuzzy information and their application to decision making," Control and Decision, vol. 22, no. 2, pp. 215-219, 2007.

[66] Z. S. Xu, "Intuitionistic fuzzy aggregation operators," IEEE Transactions on Fuzzy Systems, vol. 15, no. 6, pp. 1179-1187, 2007.

[67] Z. S. Xu and J. Chen, "An approach to group decision making based on interval-valued intuitionistic judgment matrices," System Engineering Theory and Practice, vol. 27, no. 4, pp. 126-133, 2007.

[68] Z. S. Xu and R. R. Yager, "Intuitionistic and interval-valued intuitionistic fuzzy preference relations and their measures of similarity for the evaluation of agreement within a group," Fuzzy Optimization and Decision Making, vol. 8, no. 2, pp. 123-139, 2009.

[69] S. H. Ren and K. Q. Cai, Research on Evaluation of Exploitation Feasibility and Sustainable Development Strategies for Tourism Resources in ZhouShan Sea Islands of China, Ocean Press, Beijing, China, 2010. 


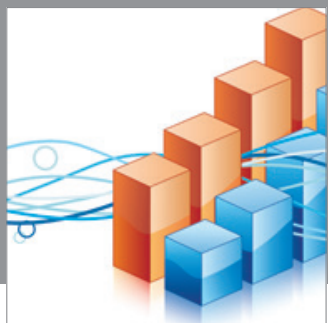

Advances in

Operations Research

mansans

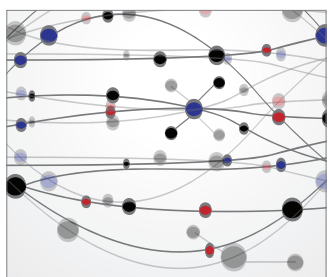

The Scientific World Journal
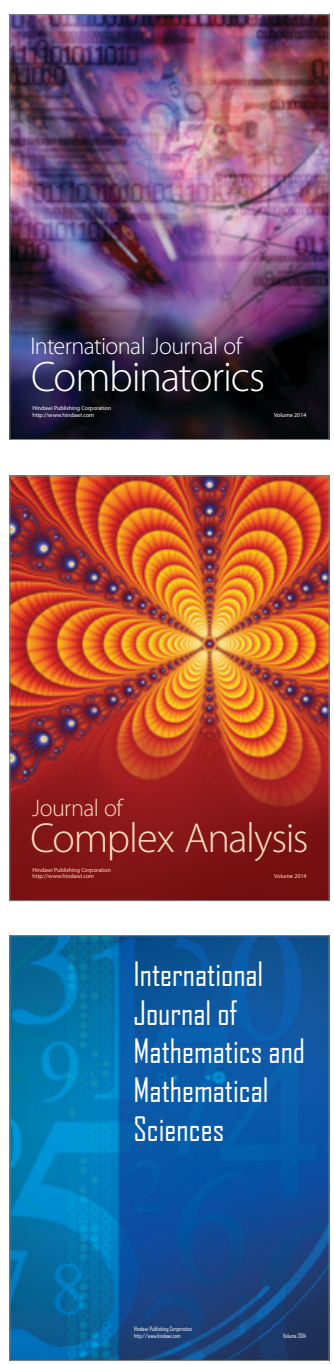
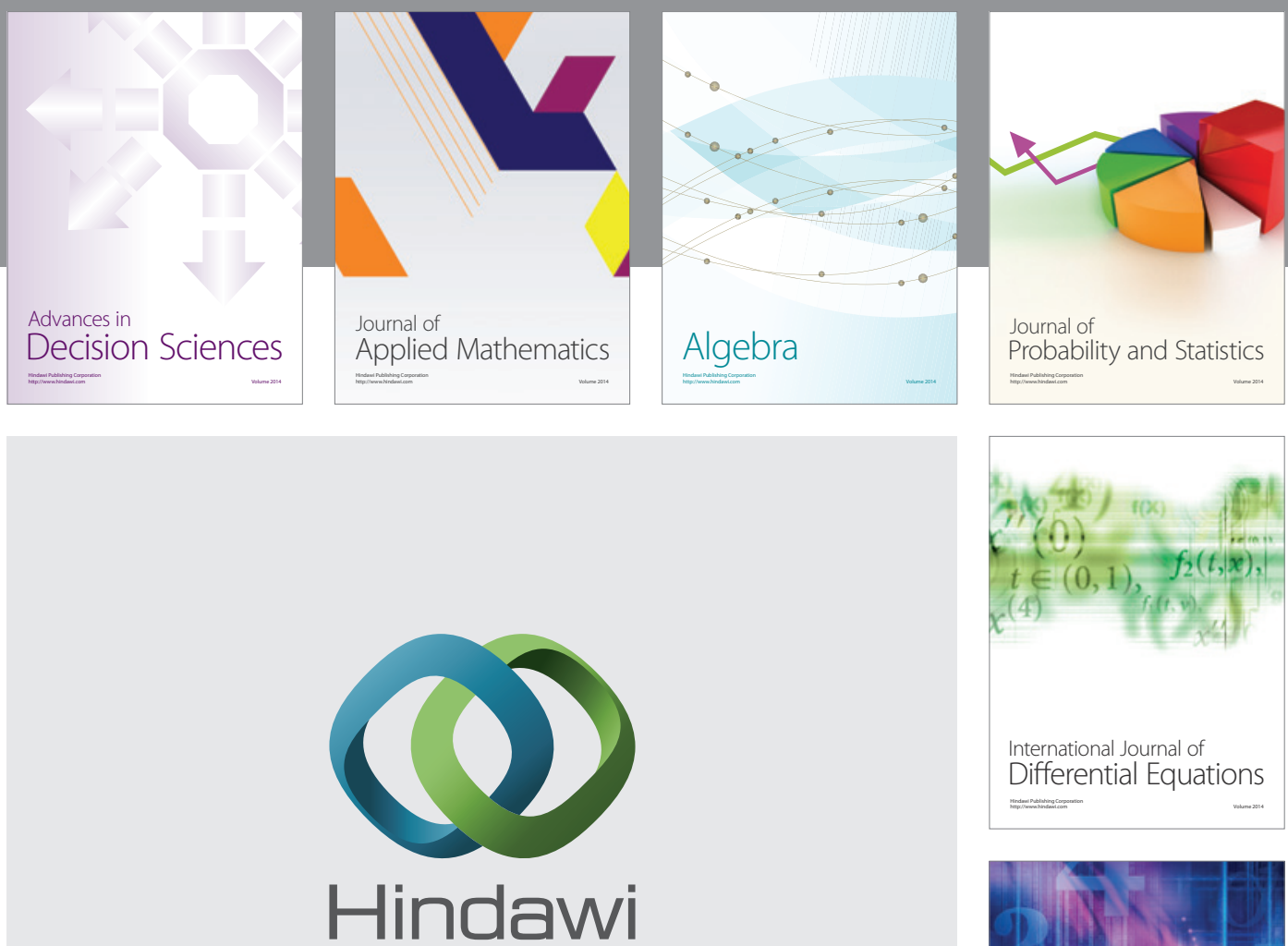

Submit your manuscripts at http://www.hindawi.com
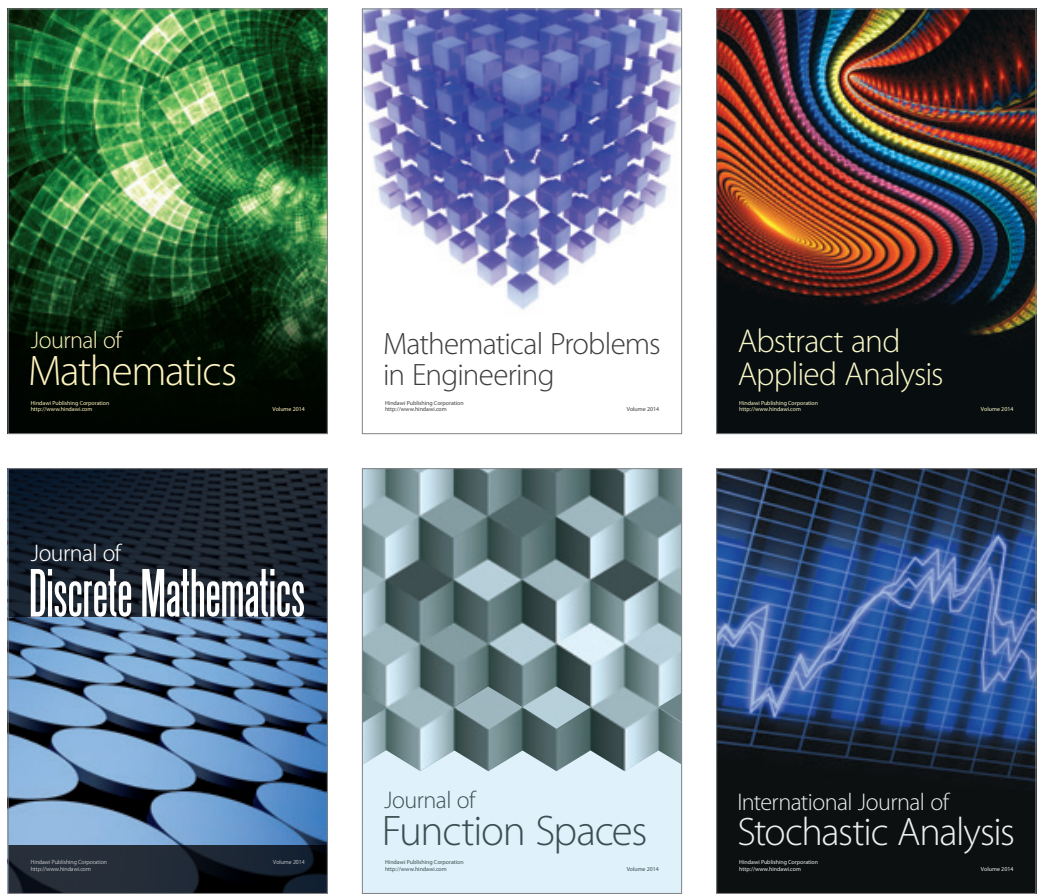

Journal of

Function Spaces

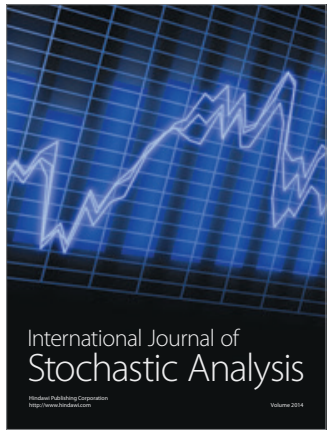

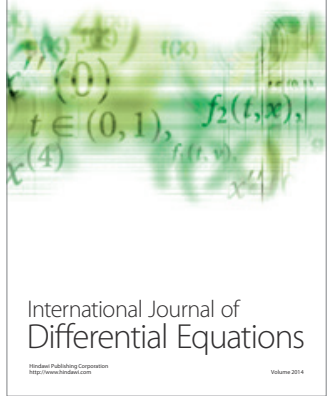
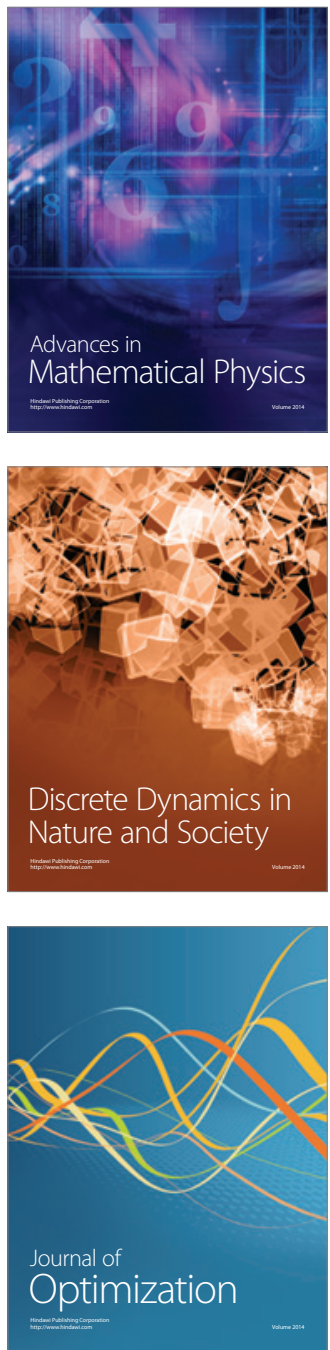